\title{
The Affective Disorder Test: a new test to assess depression and anxiety-like behaviour in rats
}

\author{
By \\ Katie Blackburne \\ A thesis submitted in fulfilment of the requirements of a Master of Science in Cognitive and \\ Behavioural Neuroscience
}

Victoria University of Wellington

2021 



\section{Table of Contents}

Abstract 1

\section{Chapter One}

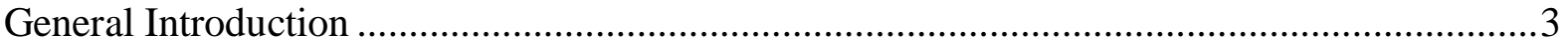

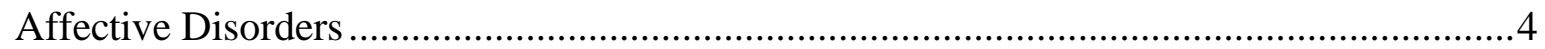

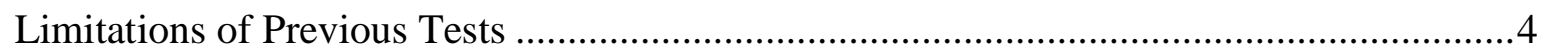

The Affective Disorder Test (ADT) …........................................................................

\section{Chapter Two}

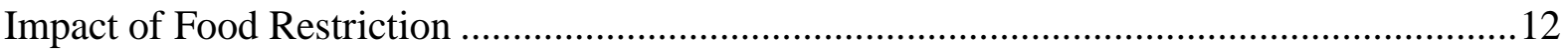

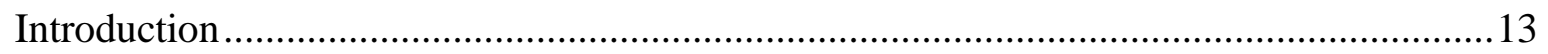

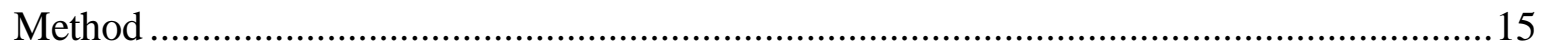

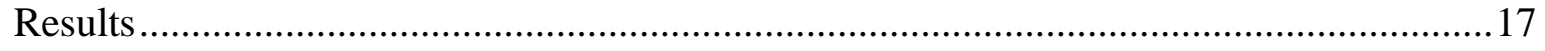

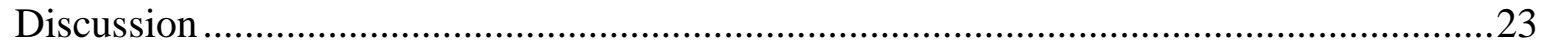

Chapter Three

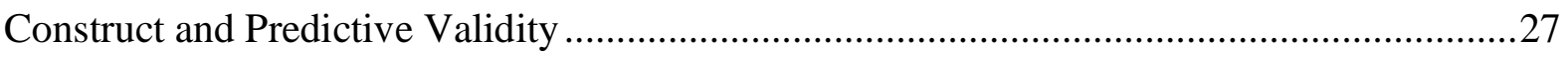

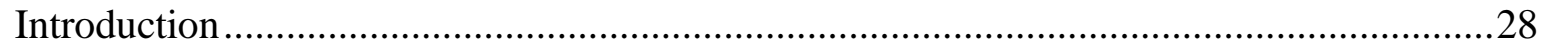

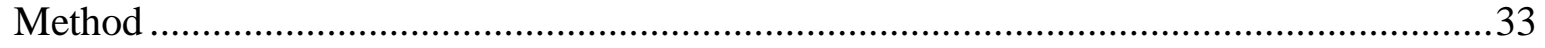

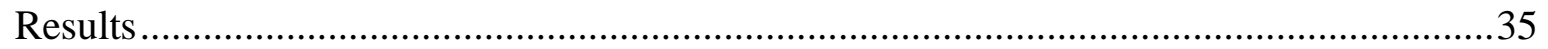

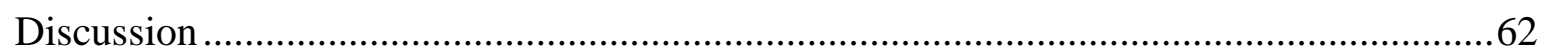

\section{Chapter Four}

General Discussion and Concluding Remarks ....................................................................69

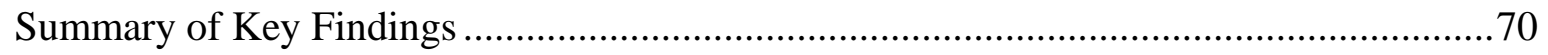

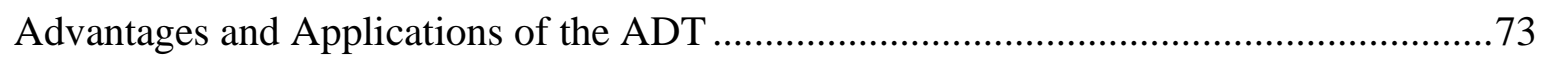

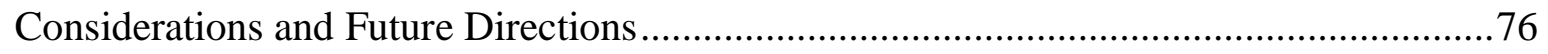

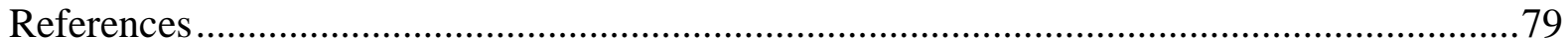

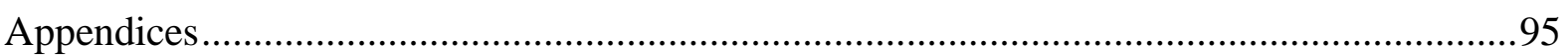


List of Figures

Figure 1. Dimensions of Successive Alleys Apparatus ....................................................

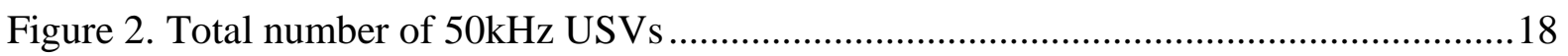

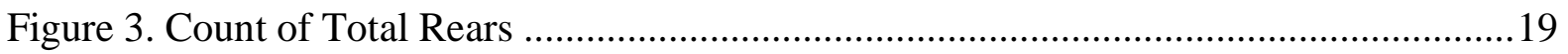

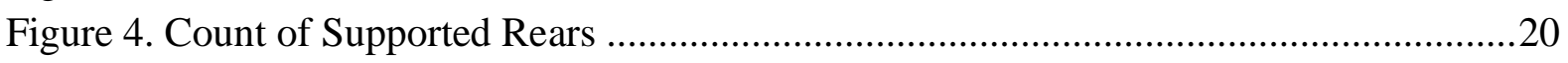

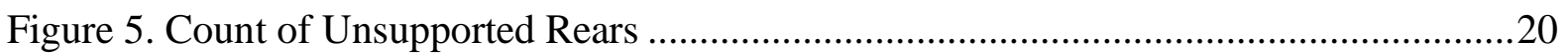

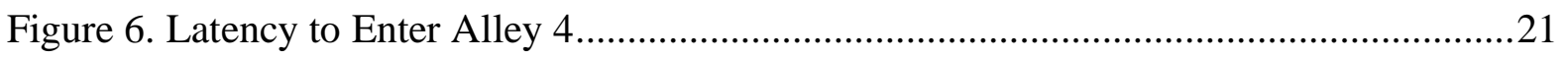

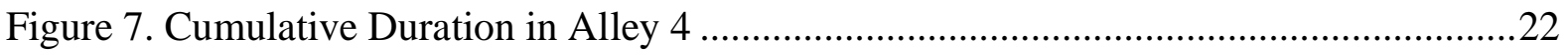

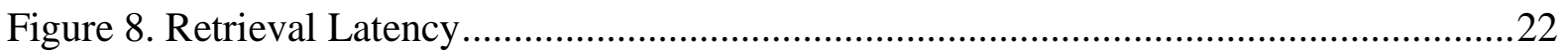

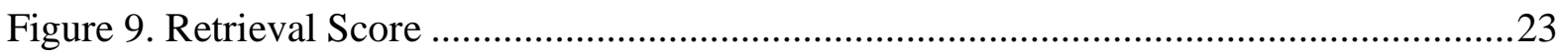

Figure 10. Genotype Differences in Proportion of 50kHz USVs .........................................36

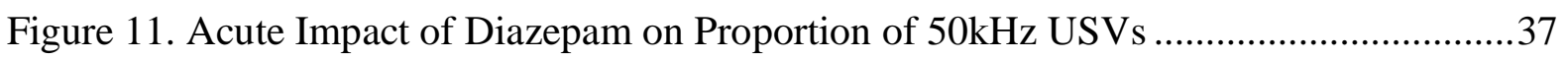

Figure 12. Long-Term Impact of Diazepam on Proportion of 50kHz USVs .........................37

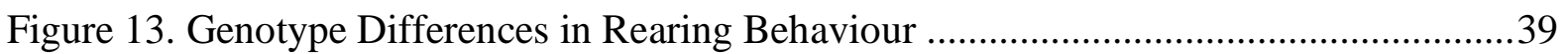

Figure 14. Acute Impact of Diazepam on Rearing Behaviour...............................................40

Figure 15. Long-Term Impact of Diazepam on Rearing Behaviour.....................................41

Figure 16. Acute Impact of Ketamine on Rearing Behaviour .................................................41

Figure 17. Long-Term Impact of Ketamine on Rearing Behaviour .......................................42

Figure 18. Genotype Differences in Cumulative Duration in Alley 4 ....................................44

Figure 19. Long-Term Impact of Diazepam on Cumulative Duration in Alley 4 ...................45

Figure 20. Long-Term Impact of Diazepam on Latency to Enter Alley 4 .............................47

Figure 21. Long-Term Impact of Ketamine on Latency to Enter Alley 4 .............................48

Figure 22. Genotype Differences in Cumulative Duration in Alley 3 ...................................50

Figure 23. Acute Impact of Diazepam on Cumulative Duration in Alley 3 ...........................51

Figure 24. Long-Term Impact of Diazepam on Cumulative Duration in Alley 3 ...................51

Figure 25. Genotype Differences in Retrieval Score ............................................................53

Figure 26. Acute Impact of Diazepam on Retrieval Score .................................................55

Figure 27. Long-Term Impact of Diazepam on Retrieval Score ..........................................56

Figure 28. Acute Impact of Diazepam on Locomotor Activity ............................................59

Figure 29. Long-Term Impact of Diazepam on Locomotor Activity ....................................60 
List of Tables

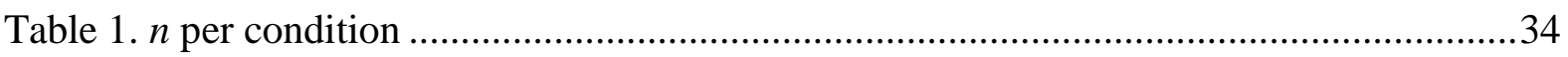

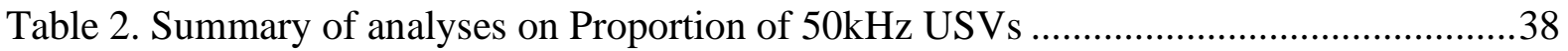

Table 3. Summary of Analyses on Rearing Behaviour........................................................43

Table 4. Summary of Analyses on Cumulative Duration in Alley 4 .....................................46

Table 5. Summary of Analyses on Latency to Enter Alley 4 ............................................49

Table 6. Summary of Analyses on Cumulative Duration in Alley 3 .....................................52

Table 7. Summary of Analyses on Latency to Enter Alley 3 ................................................54

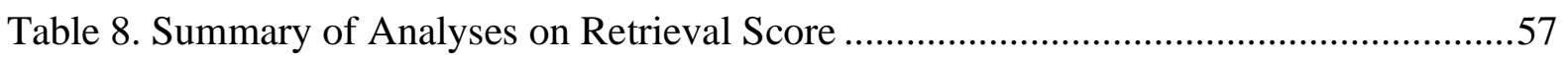

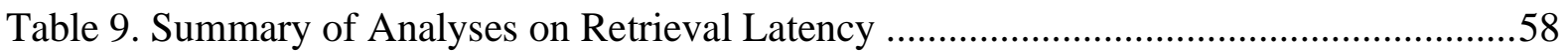

Table 10. Summary of Analyses on Locomotor Activity ..................................................61

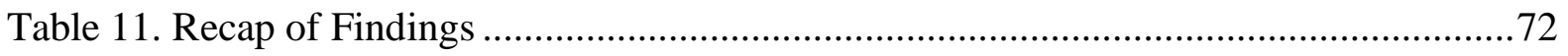


List of Abbreviations

\begin{tabular}{ll}
\hline Abbreviation & Meaning \\
\hline ADT & Affective Disorder Test \\
SAT & Successive Alleys Test \\
FST & Forced swim test \\
EPM & Elevated plus maze \\
SERT & Serotonin transporter \\
SERT ${ }^{-}$ & Serotonin transporter knockout: subjects completely \\
& lacking the serotonin transporter \\
SERT ${ }^{+} /^{+}$ & Serotonin transporter wildtypes: subjects with completely \\
& functional serotonin transporter \\
WT & Wildtype \\
USV & Ultrasonic vocalizations \\
PND & Postnatal day: indicates subjects age \\
5 -HT & Serotonin \\
SNI & Spared nerve injury \\
NMDA & N-methyl-D-aspartate \\
\hline
\end{tabular}





\begin{abstract}
Major depressive and anxiety disorders are debilitating psychiatric illnesses which are often a serious burden to the patients, their families and society as a whole. A major contributor to this burden is that a large number of patients do not respond to current therapeutics. As all treatments are established first in animal models, an imperative direction to advance treatment efficacy in humans is through progressing these models. This thesis aims to develop and validate a novel behavioural test (Affective Disorder Test: ADT) that simultaneously assesses depression and anxiety-like behaviours, as well as provides a more valid index of depressionlike behaviour than previous tests, and allows repeated testing in the same animal.

In order to develop a standardized protocol, the impact of food restriction on behaviour was examined. Subjects with ad libitum access to food, failed to exhibit sufficient responses in aspects of the test, supporting the incorporation of food restriction into the test protocol. The serotonin transporter knockout (SERT \%) rat was employed as a model of depression and anxiety, and as hypothesised displayed corresponding behaviour in the test, indicating construct validity. Finally, the predictive validity of the test was corroborated as pharmacological treatment of anxiolytic and antidepressant drugs, diazepam $(1 \mathrm{mg} / \mathrm{kg})$ and ketamine $(5 \mathrm{mg} / \mathrm{kg})$, improved subjects behaviour on relevant areas of the test. The ADT is an innovate tool which has the ability to contribute to the scientific and general community by furthering our understanding of the aetiology of these disorders and enhancing pharmacological developments.
\end{abstract}




\section{Chapter One}

Introduction to Affective Disorders and Current Tests of this Behaviour in Rodents 
Affective disorders such as major depressive and anxiety disorders are among the most severe and frequently occurring psychiatric disorders. The Global Burden of Disease study indicated that in 2017 more than 265 million people were affected by depressive disorders, and an estimated 264 million people worldwide were living with an anxiety disorder. Of additional concern, prevalence rates of both depression and anxiety are increasing at a disproportionate rate to general population growth (James at el., 2018).

Patients suffering from major depressive disorder may experience an inability to find enjoyment in previously rewarding activities, sleep and appetite disruption, fatigue and poor concentration (Beck et al., 2014). Anxiety disorders are characterized by sustained feelings of intense fear and distress, often in conjunction with physiological responses such as increased heart rate, sweating and trembling (Baxter et al., 2014).

These disorders induce serious burden for the patients across multiple domains such as physical health, education, employment and interpersonal relationships (Baxter et al., 2014), to the extent where depression has been classified as the leading cause of disability in the Western world (Friedrich, 2017).

A major problem in the care of these disorders is the lack of effective treatment. Antidepressants are amongst the most commonly prescribed drugs in OECD countries (OECD, 2019), yet despite their widespread use, current treatments present multiple shortcomings. The majority of antidepressants have a delayed onset of effectiveness which has been associated with increased suicidal ideation (Nischal et al., 2012) and discontinuation of treatment (Masand, 2003), as well as numerous aversive side effects (Ferguson, 2001). Efficacy of treatment compared to placebo appears marginal (Pigott et al., 2010), with a substantial group of patients (30-40\%) failing to show an adequate response (Al-Harbi, 2012), calling into question the efficacy of current practices and highlighting the extreme need for novel investigations.

This thesis aims to assess a novel test for depression and anxiety-like symptoms in rats which resolves the subsequently identified limitations of previously established tests, providing the research field with a valuable means of progressing our understanding and treatment of these disorders.

\section{Limitations of Previous Tests}

Whilst there are numerous animal tests for depression and anxiety, many of these have a range of limitations. First, virtually none assess both disorders within a single test. Estimates 
show that $60 \%$ of patients with anxiety will also have symptoms of depression, and the numbers are similar for those with depression also experiencing anxiety (Mathew et al., 2011). Patients with comorbid disorders often have a poorer medical prognosis and are more resistant to pharmacological treatment than patients who present with either disorder in isolation (Ballenger, 2000). Unfortunately, the majority of both animal and clinical research focuses on patients with one illness, with treatments subsequently being guided by this research. As a result, treatments for comorbid mental illnesses are lacking. This high degree of comorbidity, in conjunction with the large proportion of treatment resistant patients, indicates the importance of furthering our understanding on both recognizing and treating affective disorders when they present simultaneously.

Secondly, the validity of current tests for depression has been questioned repeatedly. The forced swim test (FST; Porsolt et al., 1977) is one of the most commonly used tests for assessing depression-like behaviour and antidepressant efficacy in rodents. The test involved placing animals in an inescapable cylinder of water and recording active (swimming and climbing) and passive (immobility) behaviours. The test was brought into widespread use due to its predictive ability of antidepressant efficacy, with effective antidepressants in humans increasing the amount of active strategies and decreasing passive strategies employed by the animal (Commons et al., 2017). Based off the observation that antidepressants reduce immobility, it was inferred that immobility is an index depressive behaviour. This immobility is described by Porsolt and colleagues (1977) as a state of "behavioural despair", when the animal realizes it cannot escape and gives up, with 'depressed' subjects 'giving up' and becoming immobile quicker than others. The tail suspension test (Steru et al., 1985) employs similar logic to the FST. Rodents are suspended by their tail for 6 minutes, and their behaviour is recorded. Initially the subject will try to escape the stressful situation by engaging in vigorous movements, however, they will eventually cease to struggle and become immobile. The duration of immobility is reduced after administration of antidepressants, therefore immobility is again considered a measure of depressive behaviour (Belovicova et al., 2017).

Despite the FST and tail suspension test showing some predictive validity of antidepressant activity via reducing immobility, the assumption that increased immobility is indicative of depressive-like behaviour is an unfounded overinterpretation. These tests lack construct validity as the dependent variable measures a subject's coping response to an acute inescapable stressor, rather than a depression-like phenotype. Many researchers argue the animal's immobility is a learned or adaptive behaviour, as opposed to reflecting a state of despair. Typically, a subject is exposed to the test twice, prior and post administration of a 
substance, thus immobility may become a learned behaviour. Subjects that were administered anisomycin, a drug which interrupts the consolidation of memories, showed no difference in behaviour on the first test, but increased mobility compared to controls on the second test, accentuating the role learning processes have on this behaviour (De Pablo et al., 1989). Molendijk and de Kloet (2015), posit that immobility likely reflects an adaptive behaviour. Where swimming and climbing expends excessive amounts of energy, subjects who become immobile quicker as a means of conserving energy have a greater chance of survival if submerged for an extended period of time. When exposed to the test until sinking, immobility in the first 15 minutes predicted this outcome, with more mobile subjects sinking quicker compared to those who conserved energy and were immobile (Nishimura et al., 1988).

Therefore, immobility could be interpreted as the application of cognitive functions such as learning and memory resulting in adaptive coping strategies to enhance survival, insinuating the need for an alternative test of depression-like behaviour.

Furthermore, these tests induce significant amounts of stress and discomfort in the animals, which is critical to consider in an ethical context. The 3Rs (replacement, reduction and refinement) of animal research, have long been recognised as the best practice for ensuring a high standard of ethical treatment is applied when using animals in scientific procedures (Kirk, 2018). The principle of refinement refers to adopting methods which minimise suffering and distress to animals. As stress is intrinsic to tests such as the FST and tail suspension test, there is an evident need for a more refined test for studying aspects of depression.

A final limitation is that particularly when it comes to tests of anxiety-like behaviour, virtually all suffer from what is called the one-trial-tolerance effect. The most common test for anxiety is the elevated plus maze (EPM), with over 2,000 papers related to this test being published over the past 20 years (Walf \& Frye, 2007). This test involves placing animals in the centre of a four-arm maze, consisting of two open arms and two arms with high walls, and recording their behaviour for 5 minutes.

A rodent's behaviour in this task relies on the natural conflict between their preference for protected areas (closed arms) and their innate motivation to explore novel environments (entire maze, including open arms). Behaviours typically recorded in this test are the time spent and entries made on each arm, with anxiety-like behaviour being operationalized by less time spent on the open arms of the maze. The test shows robust predictive validity, with anxiolytic treatment consistently increasing time spent in open arms compared with vehicle controls.

The 'one-trial tolerance' was first demonstrated by File et al. (1990), who found that prior exposure to the maze eliminated the effects of anxiolytics. Extensive research has since 
been conducted to explore the mechanisms behind this phenomenon. Pre-exposure to the maze, both in the presence of anxiolytic treatment and untreated, markedly reduced the amount of time subsequently spent on open arms, suggesting the one-trial tolerance is not driven by tolerance to the effect of treatment (Treit et al., 1993). Rogers and Shepherd (1993) hypothesised that the tolerance may be explained by a motivational deficit upon second exposure. This hypothesis is supported by findings that pre-exposure resulted in a significant reduction in locomotor activity, indicating habituation to the maze and a reduction of exploratory behaviour (Dawson et al., 1994). Furthermore, the introduction of an aversive stimuli in the closed arms during the second trial restored the anxiolytic effect of benzodiazepines (Pereira et al., 1999). These findings indicate that whilst on first exposure to the test subjects are faced with a motivational conflict, this exploratory behaviour has habituated by trial 2 and subjects will remain in the preferred closed arms as they have no reason to explore the open arms.

Other tests of anxiety such as the open field test and light/dark box are based off the same principle of conflict between exploration and eversion to open areas. Therefore, although much less research has been done with these tests, they likely also suffer from the same one trial tolerance phenomenon.

The one-trial effect poses multiple problems. Firstly, in humans, anxiety symptoms are long lasting, a facet of anxiety which cannot be captured by these tests. Secondly, it makes it impossible to test different dosage effects or treatments in the same animals, increasing the number of animals required for testing dramatically. Thus, there is an urgent need for an improved test that can repeatedly assess anxiety, allowing the measurement of a prolonged anxious state in the same animal, and reduce the number of animals needed to assess different treatments.

The Affective Disorder Test (ADT)

To overcome the caveats of established tests outlined above, I propose a novel test, the Affective Disorder Test (ADT). The ADT will allow the assessment of depression and anxietylike symptoms in the same animals, induce substantially less distress than current tests for depression, and can assess symptoms repeatedly.

My proposed test is an adaptation of the Successive Alleys Test (SAT) (Deacon, 2013). The SAT is an apparatus consisting of four segments (alleys) in a straight line. Each segment 
has progressively lower walls, and becomes more narrow and lighter, increasing the anxiogenic properties of each alley (see figure 1).
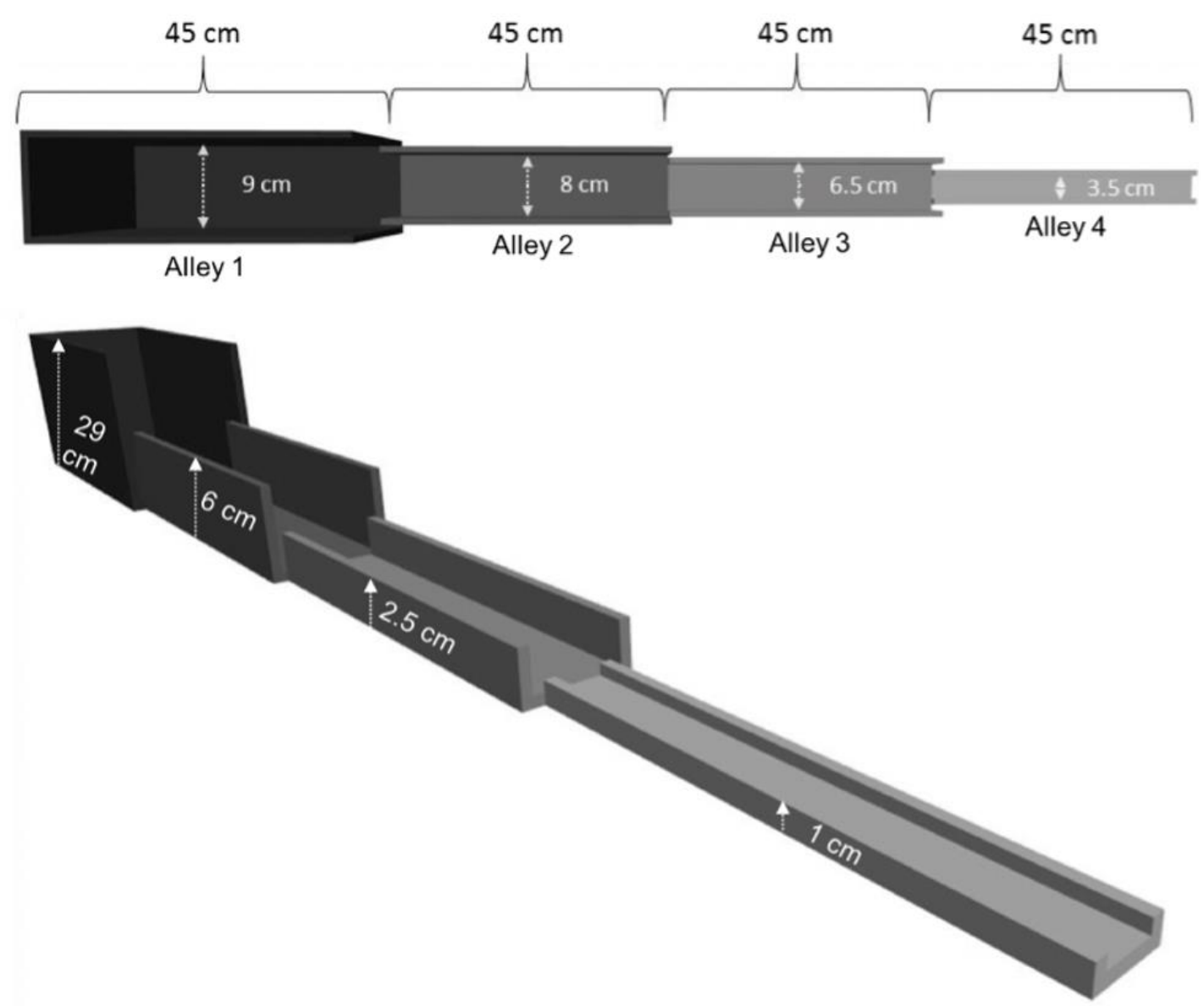

Figure 1. Dimensions of Successive Alleys Apparatus

While this test has some advantages over the elevated plus maze (more graded increase in anxiogenic properties, removal of the ambiguous central area between the open and closed arms), it still suffers from the one-trial tolerance phenomenon, with repeated testing reducing time spent in more anxiogenic alleys (unpublished data from our laboratory).

Food rewards are widely used in animal research as a means of motivating the subject to perform a task, therefore, to counter the reduced motivation evident after repeated exposure, my test incorporates a food reward (half a frootloop) placed in the centre of each alley. The introduction of food will restore a reward/safety conflict, with the aim of continually motivating subject to enter the anxiogenic areas on each trial. Similar to the SAT and previous tests of anxiety, latency to enter and duration spent in each alley are measured as an index an anxiety. The addition of bait in each alley also allows for the measurement of a novel 
parameter: food retrieval behaviour. Retrieval scores are calculated based off the alley each frootloop is eaten in, with alleys being allocated a score of 1 - 4 (alleys $1-4$ respectively). If a subject retrieves all 4 frootloops from their baited position and returns to alley 1 to eat them, they would receive a score of 4 , whereas a subject who consumes each frootloop in the alley it was presented would score 10. Essentially, the alleys higher in anxiogenic properties are attributed higher scores, therefore, a higher retrieval score indicates subjects are consuming frootloops in more anxiogenic alleys, inferring lower levels of anxiety.

In addition to providing a novel measure of anxiety, the integration of food reward to the test allows for the measurement of anticipatory pleasure. As the validity of current tests for depression have been questioned, it has been suggested that a more valid measure of this behaviour may be the assessment of anticipatory pleasure. Anticipatory pleasure can be assessed in animals by repeatedly pairing a specific environment with a future food reward. As subjects learn to expect food when placed in the corresponding area, they show anticipatory responses, typically indexed as an increase in rearing behaviour (Makowska \& Weary, 2016).

Translating to the human condition, one of the major diagnostic criteria for depression is anhedonia, defined by a decreased interest and pleasure in activities (Thomsen, 2015). Anhedonia can be further conceptualized as a deficit in anticipatory pleasure (the anticipation of a reward eliciting pleasure) or consummatory pleasure (receiving the reward eliciting pleasure) (Rizvi et al., 2016). There is growing evidence that anhedonia experienced by patients suffering from depression is primarily driven by a deficit in anticipatory pleasure (Treadway et al., 2012; Thomsen, 2015; Whitton et al., 2015). This is supported by animal models of depression showing reductions in rearing behaviour during anticipatory pleasure paradigms, implying a deficit in this pleasure response (Makowska \& Weary, 2015).

A limitation of indexing anticipatory pleasure purely via rearing, is that this measure relies on locomotor activity and may be confounded by manipulations that alter this, such as pharmacological treatment. Therefore, the ADT will also measure ultrasonic vocalisations (USVs) as a second index of this anticipatory response. USVs are widely recognised as a means of communication between rats (Takahashi et al., 2010). However, they are also emitted in non-social contexts, suggesting they may also be used as a tool to study emotional state (Simola \& Granon, 2019). Rats emit USVs of differing frequency and rate in a variety of situations. Rats typically produce calls at a $50 \mathrm{kHz}$ frequency (true range: $30-90 \mathrm{kHz}$ ) and shorter call rate in pleasant situations, and longer calls in a $22 \mathrm{kHz}$ frequency (true range: $18-30 \mathrm{kHz}$ ) in aversive situations (Saito et al., 2019). Previous literature has shown that 50kHz USVs are elicited when rats are exposed to cues that predict rewarding stimuli such as food or drugs of abuse (Buck et 
al., 2014). Thus, 50kHz USVs may be assessed as an additional index of anticipatory response, with a reduction in calls implying anhedonia, a depression-like symptom.

To assess anticipatory pleasure, subjects will be placed in a small enclosure (anticipation box) for 5 minutes before placing them in the SAT. As they learn to associate this enclosure with receiving food, the anticipation of this reward will be measured by rearing behaviour and $50 \mathrm{kHz}$ USVs. Focusing on the reduction in positive affect (i.e. reduced anticipation of reward) rather than the presence of negative affect (behavioural despair) as seen in the forced swim test, may result in a more refined test of major depressive disorder. Incorporating the anticipation box into the test will allow for the assessment of depression and anxiety-like symptoms in the same test, improving our ability to research these disorders when they present comorbidly.

The current thesis aims to establish a sound protocol and validate the ADT. The validity of animal models is typically evaluated through three key criteria: predictive, face, and construct validity. Predictive validity refers to how well a model can be used to predict currently known aspects of the human disease and is widely discussed in terms of responsiveness to treatments. Essentially, this means if a treatment is effective in clinical populations, this should have a parallel result in the animal model. It is crucial here to also consider the treatment conditions. For example, if a treatment is found to be effective after chronic but not acute administration in a human population, it should also be ineffective after single administration in the animal model, and only show efficacy after chronic administration.

Face validity refers to the phenomenological similarities between the presentation of the disorder in the human condition and in the animal model. This is often established by testing whether animals behaviour in the test resembles the symptomology of the human condition. Relying on rodents' unconditioned fear of heights and open spaces, the elevated plus maze is widely recognised as having strong face validity. Subjects in this test show consistent avoidance of the open arms and spend the majority of their time in the enclosed areas of the maze, behaviour which is expected of an anxious phenotype.

Lastly, construct validity is based around the theoretical rationale of the model. Appropriate mapping between the hypothesized mechanisms underlying the human condition must be considered and translated to the animal model, such as vulnerability genes, neurobiological mechanisms, and environmental factors.

The ADT likely possesses strong face validity. It relies on the same exploration/safety conflict as the EPM to measure anxiety-like behaviour, with previous research in our laboratory showing subjects spend less time in the open compared to closed alleys. It also indexes 
depression-like behaviour through anhedonia, a symptom which is strongly equated to the presentation of this disorder in a human population. The aim of the present thesis is therefore to assess the predictive and construct validity (chapter 3). Construct validity will be assessed by employing an established animal model of depression and anxiety, the SERT knockout rat. Predictive validity will be assessed by investigating the impact of diazepam, a well-established anxiolytic treatment, diazepam (Pringle et al., 2016), and ketamine, a drug which has been shown to produce antidepressant effects (Zarate et al., 2006).

However, before assessing the validity of the ADT, the basic premise that a food reward is a strong enough incentive to overcome the one-trial-tolerance and induces an increase in rearing and $50 \mathrm{kHz}$ USVs needs to be assessed. Accordingly, experiment one, outlined in chapter 2, aims to investigate whether food restriction of subjects is necessary for these responses to occur, or whether highly palatable food (like frootloops) alone is rewarding/motivating enough. 
Chapter Two

Investigation of the Impact of Food Restriction 
Food restriction is commonly used in animal research as a means of encouraging subjects to learn or perform behaviours, by increasing motivation to work for a food reward (Koote et al., 2009).

Previous work using the ADT in our laboratory has shown that when subjects are restricted to $85-90 \%$ of their free feeding weight, they spent more time in alley 4 (most anxiogenic alley) as the number of trials progressed (Kidwell, 2019). When food restricting subjects to $95 \%$ of their free feeding weight, O'Connell (2020) also found an increase in the duration spent in alleys 3 and 4 across trials. These findings indicate this test does not suffer from the one-trial tolerance when subjects are food deprived. Furthermore, subjects restricted at the $95 \%$ level, also showed an increase in rearing behaviour and 50kHz USV's emitted from baseline to day 6 of testing, evidence of an anticipatory response (O'Connell, 2020). These findings support the ability of the ADT to produce anticipatory responses and remove the onetrial tolerance, when subjects are food restricted.

However, a key consideration in experimental design is determining whether offering palatable rewards can achieve the desired behavioural performance without the use of restriction. In an overview of food and water restriction protocols, Toth and Gardiner (2000), note that if a task is relatively simple (e.g. a single lever press) and the subject has prior experience with the task, then palatable fluid such as sucrose solution can be reinforcing enough to motivate the subject to perform the task without restriction. Conversely, it has been found that even when provided access to a highly palatable reward, if subjects are not food restricted, they take longer to learn to do the task, perform the task poorly, or in some cases do not perform at all (Hughes et al., 1994; Kant et al., 1986). Furthermore, when investigating the rewarding value of sucrose pellets with a conditioned place preference paradigm, place preference was observed for food-restricted, but not ad-libitum fed rats (Figlewicz et al., 2001). This effect was found across a range of different doses of pellets, indicating that the palatable sucrose pellets were not considered a valuable reward when subjects were not food restricted.

The degree of restriction necessary to achieve reliable performance and detect effects is also crucial to consider. The general consensus is that the degree of restriction necessary to encourage reliable performance is heavily influenced by the nature of the task, with complicated tasks and more demanding reinforcement schedules typically requiring more rigorous restriction protocols (Toth \& Gardiner, 2000). In a two-choice visual discrimination task, mice maintained at $80 \%$ of free feeding weight showed improved performance on this task compared with counterparts maintained at $90 \%$ weight. Most importantly however, genotype effects on learning strategy were only detected under the more severe level of food 
restriction (Makowiecki et al., 2012), highlighting the importance of establishing the degree of restriction required to reveal effects.

Food restriction has been reported to have various effects on cognitive processes such as learning and memory (Rajab et al., 2014; Roberge et al., 2008), and is associated with alterations to neurochemical activity such as dopamine and serotonin neurotransmission (Li \& France, 2008; Sevak et al., 2008). These effects are inconsistent across the literature, potentially due to differences in subjects used (sex, age, strain) and the restriction protocol employed, warranting consideration prior to incorporation into a test protocol.

Discrepancies on the impact of food restriction on behaviour have also been reported. After acute food restriction, male adult rats exhibited a decrease in anxiety-like behaviour on numerous measures such as the elevated plus maze, open field test and evoked beam walking test (Selakovic \& Joksimovic, 2014). A contrasting pattern of results was found with chronic food restriction of young adolescent rats. Male Sprague-Dawley rats that were subject to five weeks of food restriction showed increased immobility in the FST and spent more time in the closed arms of the elevated plus maze, indicating depression and anxiety-like behaviour (Jahng et al., 2007). As the ADT measures depression and anxiety-like behaviour, the differential impact of acute and chronic food restriction may introduce a confounding variable, therefore should only be executed if necessary.

In addition, it is unclear how stressful food restriction regimes are to the subjects. An assessment of subject's welfare indexed by levels of activity, look/posture, urine/feces, and body condition, concluded that food restriction at the $85 \%$ level had no impact on the subject's wellbeing (Goltstein et al., 2018). However, studies have shown an increase in corticosterone levels after 2 (Toth \& Gardiner, 2000), 8 (Marinelli et al., 1996), and 37 days of food restriction (Heiderstadt et al., 1999). Increased ccorticosterone levels have repeatedly been found in rodents subject to different forms of stress (Lapointe et al., 2015; Thomas et al., 2006). These findings indicate food deprivation may induce a certain level of stress in the subject (although certainly much less than the FST or tail suspension test). The ADT aims to improve on previous tests in numerous ways, one of which is by measuring depression-like behaviour in a way which induces less stress to the subjects. Therefore, if food restriction is stressful in itself, it is crucial to consider whether this is a necessary component of the test.

The effects of food restriction on numerous factors may vary to a great extent, therefore, in order to define a standardized protocol which can be used by the wider scientific community, it is crucial to examine the impact of this parameter on behaviour in the ADT. 
Based on the vast amount of literature supporting the use of food restriction as a means of increasing subject motivation, and previous research with the ADT, it was hypothesized that food restriction would be required to capture appropriate anticipatory pleasure responses, and continuously motivate subjects to enter anxiogenic alleys. That is, it was predicted that free feeding subjects will show reduced rearing behaviour and $50 \mathrm{kHz}$ USVs. In addition, it was predicted that free feeding subjects would spend less time in alley 4, whilst taking longer to first enter this alley, and display lower retrieval scores.

\section{Method}

\section{Subjects}

A cohort of 20 male Sprague-Dawley rats were used in this first experiment. All animals were housed in individually ventilated polycarbonate cages ( 2 per cage) in a temperature $\left(21^{\circ} \mathrm{C} \pm 2\right)$ and humidity $(55-60 \%)$ controlled environment. To ensure testing occurred during active periods, animals were housed on a reverse light cycle (lights on 19000700 hours).

Subjects were tested in adulthood, ranging from age postnatal day (PND) 60-82. Half of the subjects were food restricted and the others had ad libitum access to food ( $n=10$ per condition). Food restricted animals were weighed daily and maintained at $85-90 \%$ of their free feeding weight. This weight was achieved by giving subjects $12-15 \mathrm{~g}$ of food daily in the evening for one week prior to testing. The testing order of animals was counterbalanced to ensure variable delays between the end of test sessions and being fed, with a minimum of a 1.5-hour delay.

All animals were bred and housed in the Victoria of University vivarium in accordance with the Animal Welfare Act (1999), and all procedures were approved by the VUW Animal Ethics committee (AEC 27328).

\section{Experimental Setting}

The alley was constructed using gloss black polycarbonate panels slotted together to create the complete unit. Each alley was $45 \mathrm{~cm}$ in length $(180 \mathrm{~cm}$ total), with the wall height and alley width reducing progressively to create an increase in the anxiogenic properties of each 
alley (see figure 1). The anticipatory box was assembled from four black polycarbonate sheets to create a $40 * 40 * 40$ open-air chamber.

The alley apparatus was positioned in the centre of the experimental room, with the anticipation box located beside the alley. An UltraMic microphone (250K 16 bi; Java Sound) was attached to an adjustable pole mount and set beside the anticipatory box, elevated enough so animals could not reach it when rearing. A USB camera was mounted above the experimental area, to provide a birds-eye view of both arenas.

A lamp was positioned beside the anticipatory box to ensure the arena was evenly lit at 25 lux. The lamp was turned off once animals were placed into the successive alley. Dim LED lighting was used to main approximately, 1, 5, 10 and 15 lux in alleys $1-4$ respectively.

\section{Procedure}

For 5 consecutive days prior to the actual test, animals were habituated to the anticipatory box, to reduce novelty-induced exploration. Subjects were transported from the housing room to the experimental room in their home cage, one cage at a time, and habituated to the room for 10 minutes. Next, each animal was handled individually for 2 minutes, and then placed in the anticipatory box for 5 minutes. USVs were collected using the UltraMic microphone and rearing behaviour was scored manually. Rearing behaviour was categorized into "supported" where the animal raised both front paws off the ground and placed them against the walls of the arena, and "unsupported" where the animal raised both front paws off the ground but did not lean them against the wall. If only a single paw was raised off the ground, this was not counted as a rear. Likewise, if only one paw was placed on the wall, this did not meet the characterization of a "supported" rear and was classified as "unsupported". A single rearing behaviour ended when the animal placed both front paws back on the ground. The order of habituation was counter-balanced across all animals.

In these 5 days animals were also given frootloops (time-locked with feeding of food restricted animals) in their home cage to ensure they developed a preference for them. Three frootloops were halved and dispersed across the cage floor where they were easily accessible to both animals in the cage. The experimenter lingered to observe and ensure frootloops were consumed by both subjects.

Testing occurred for six consecutive days, beginning immediately after the last day of habituation. During testing days, animals were transported and habituated to the experimental room as they were during the habituation period. Following the 10 minutes of habituation to 
the experimental room, animals were placed into the anticipation box for 5 minutes. During this time USVs and rearing behaviour were recorded as outlined in the habituation procedure. The subject was removed from the box and placed in the centre of alley one, facing the back wall of the alley. Activity was recorded for another 5 minutes using the overhead camera. The latency to enter each alley and the duration spent in each alley was calculated using Ethovision software, while latency to consume the first frootloop and the location of consumption was manually recorded. The location of frootloop consumption was used to calculate a 'retrieval score' for each subject. Each alley was assigned either one, two, three or four points (corresponding to alley $1-4$ respectively). The number of frootloops consumed in each alley was then multiplied by these points and summed to obtain a final retrieval score. For example, if all four frootloops were consumed in alley one, the animal would receive a score of 4 . If animals did not consume any frootloops, they were assigned a latency score of 300s and retrieval score of 0 . As alleys higher in anxiogenic properties are attributed higher points, a higher retrieval score indicates a lower level of anxiety. The anticipation box and alleys were wiped clean between each session with Virkon ${ }^{\circledR}$, and alleys were re-baited with half a frootloop in the centre of each alley for the next animal.

\section{Statistical Analyses}

All results were analysed using a 6 (day: 1-6) x 2 (condition: free feeding, restricted) repeated measures mixed ANOVA, with day as a within-subjects factor and condition as a between subjects factor. Two trials were removed from USV analysis due to file corruption.

\section{Results}

\section{Anticipation Box}

\section{USVS}

To examine the effect of day and feeding condition on anticipatory response, the total number of $50 \mathrm{kHz}$ USVs emitted across the 6 days of testing was assessed (see figure 2). For the effect of day, Mauchly's test of sphericity was violated, therefore a Greenhouse-Geisser correction was used. Results showed a significant main effect of day $[F(1.87,29.89)=5.13, p$ $=.014]$, and a significant main effect of condition $[F(1,16)=6.80, p=.019]$, whereby the 
number of 50kHz USVs emitted increased across the days of testing, and the food-restricted condition emitted more $50 \mathrm{kHz}$ USVs on average.

Results also showed significant interaction between day and condition $[F(1.87,29.89)$ $=5.17, p=.013]$. Post hoc comparisons using the Bonferroni correction to adjust for multiple comparisons indicated that there was no significant difference between the groups in the number of $50 \mathrm{kHz}$ calls made on days $1-5$, however the food restricted condition emitted significantly more calls on day 6 than the free-feeding condition $(p=.021)$.

Figure 2. Total number of 50kHz USVs

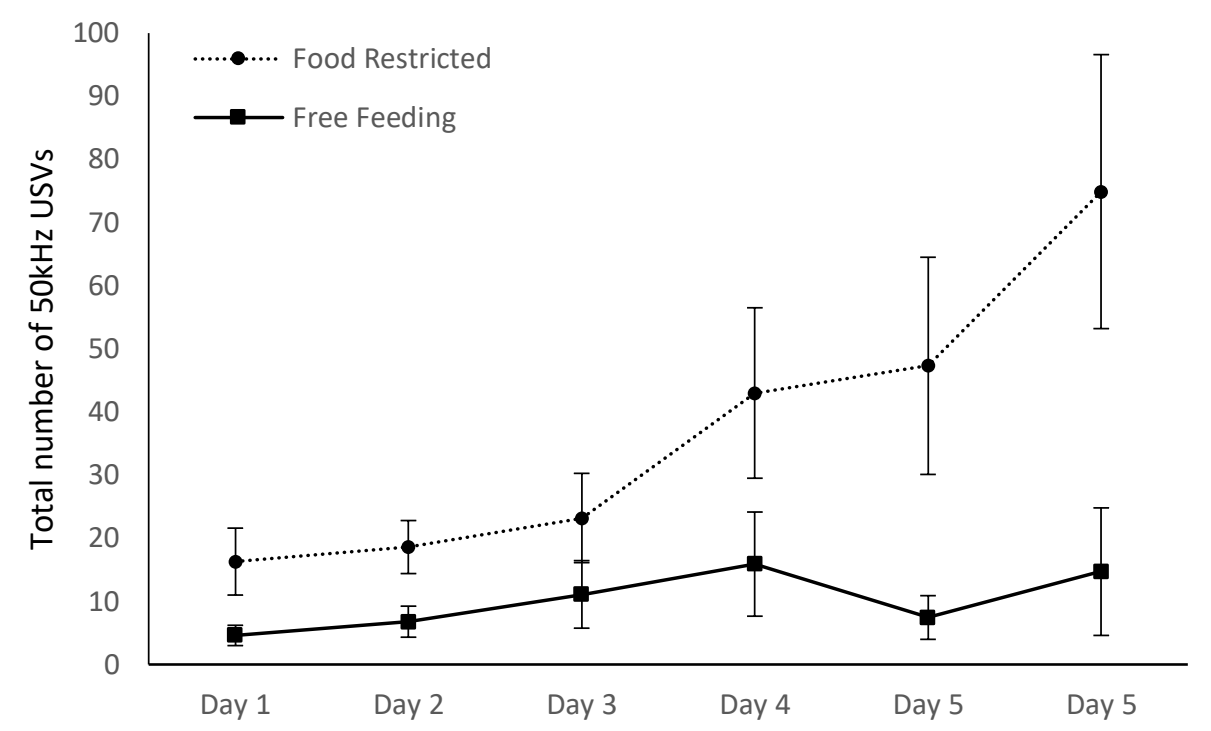

Figure 2. Means and \pm SEM of the total number of $50 \mathrm{kHz}$ USVs emitted in the anticipation box on each day of testing. Consecutive days are presented on the $x$ axis, and the total number of $50 \mathrm{kHz}$ USVs is depicted through the $y$ axis.

\section{Rearing}

To further evaluate the impact of food restriction on anticipatory pleasure, rearing behaviour in the anticipation box was also assessed. A significant main effect of day $[F(5,90)$ $=40.93, p<.001]$, and condition $[F(1,18)=32.5, p<.001]$ was found, such that total rearing increased across days, and the food-restricted condition reared more compared to the freefeeding subjects (see figure 3).

Results also revealed a significant day*condition interaction $[F(5,90)=5.09, p<.001]$. Follow up post hoc analysis applying a Bonferroni correction showed no significant difference between conditions on days 1 and 2, however significantly more rears were recorded for food 
restricted subjects compared to free feeding subjects on day $3(\operatorname{adj} p=.007)$, day $4(p=.018)$, day $5(p<.001)$, and day $6(p=.006)$.

Figure 3. Count of Total Rears

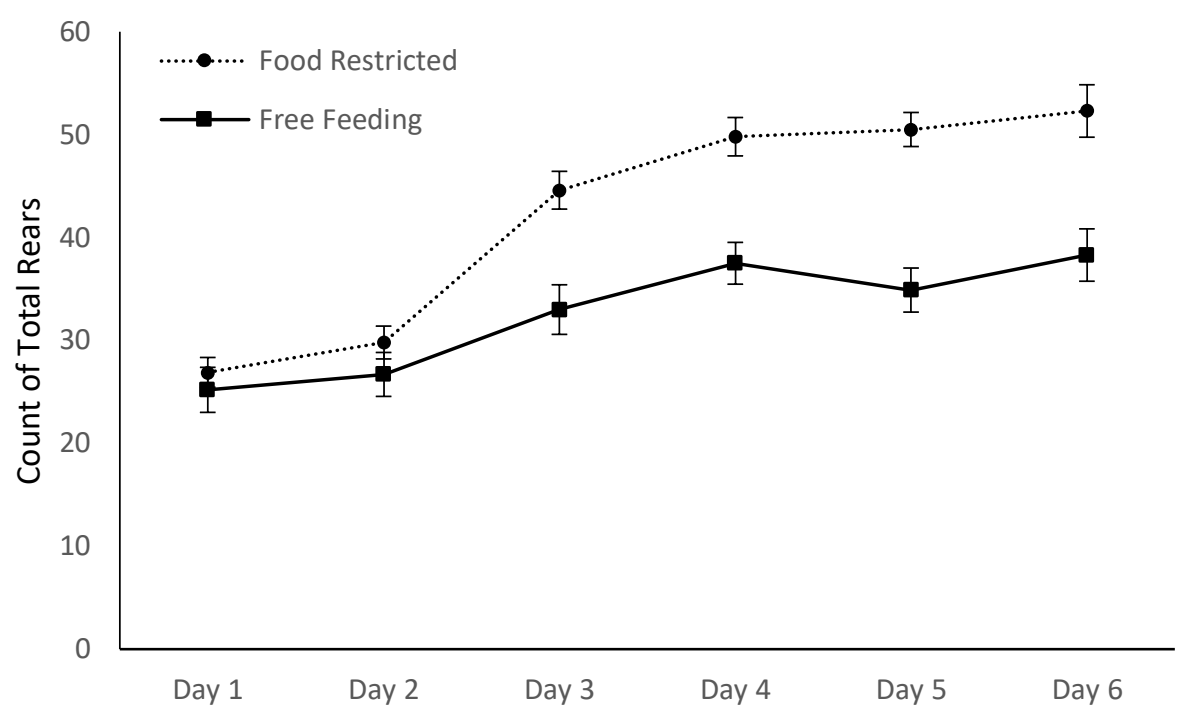

Figure 3. Means and \pm SEM of the total number of rears made in the anticipation box on each day of testing. Consecutive days are presented on the $x$ axis, and the total number of rears counted is depicted through the $y$ axis.

Further analysis examined the two different categories of rearing separately. Previous research indicates that unsupported rearing is more indicative of anticipation than supported rearing (Sturman et al., 2018), therefore this separate analysis can provide further insight into the basis of the behaviour.

Analysis of supported rearing revealed a significant main effect of day $[F(5,90)=$ $18.27, p<.001]$, and condition $[F(1,18)=9.57, p=.006]$, indicating that supported rearing increased across the days of testing, with the food restricted condition rearing more overall (see figure 4). No significant interaction between day and condition was found.

Unsupported rearing also showed a significant main effect of day $[F(5,90)=23.02, p$ $<.001]$, and a significant main effect of condition $[F(1,18)=11.80, p=.003]$. Furthermore, a significant day*condition interaction was found $[F(5,90)=3.96, p=.003]$ (see figure 5). Follow up Bonferroni post hoc comparisons showed that there was no significant difference in the number of free rears between groups on days 1-3, and day 5, however the food restricted condition made significantly more rears than the free feeding condition on day 4 ( $p=.017)$ and $6(p=.050)$. 
Figure 4. Count of Supported Rears

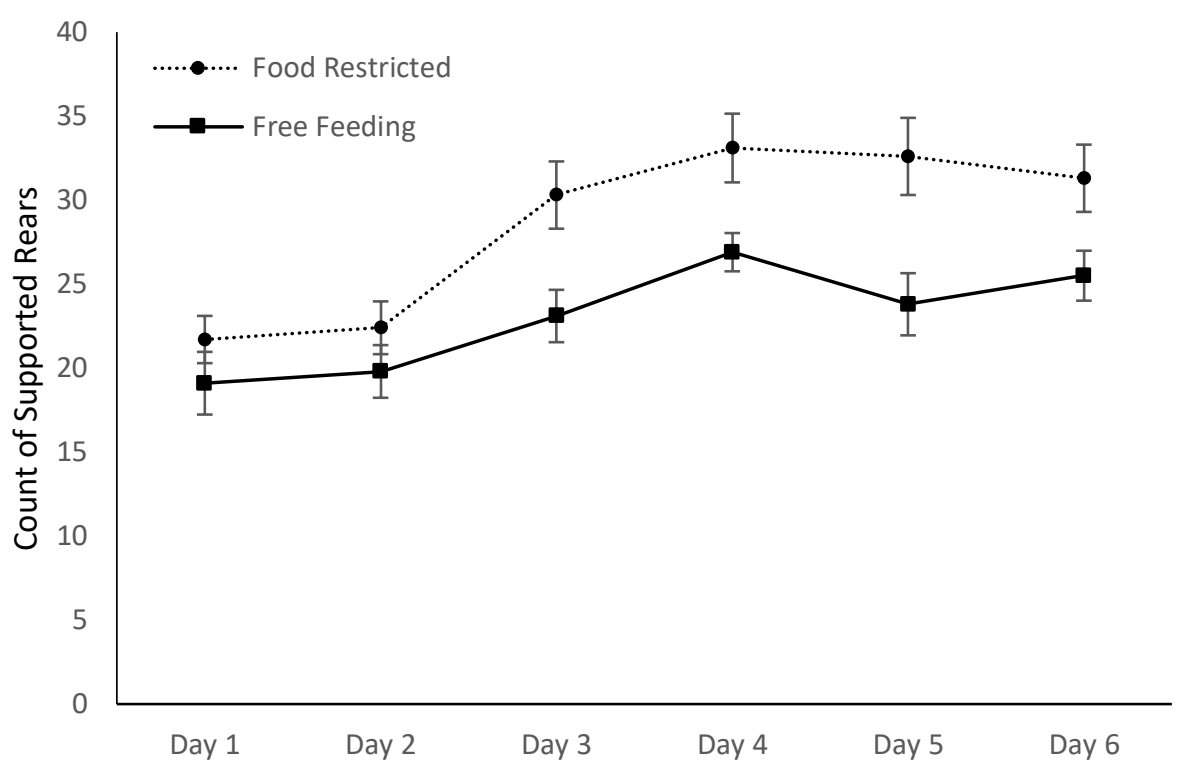

Figure 4. Means and \pm SEM of the number of supported rears made in the anticipation box on each day of testing. Consecutive days are presented on the $x$ axis, and the count of supported rears is depicted through the $y$ axis.

Figure 5. Count of Unsupported Rears

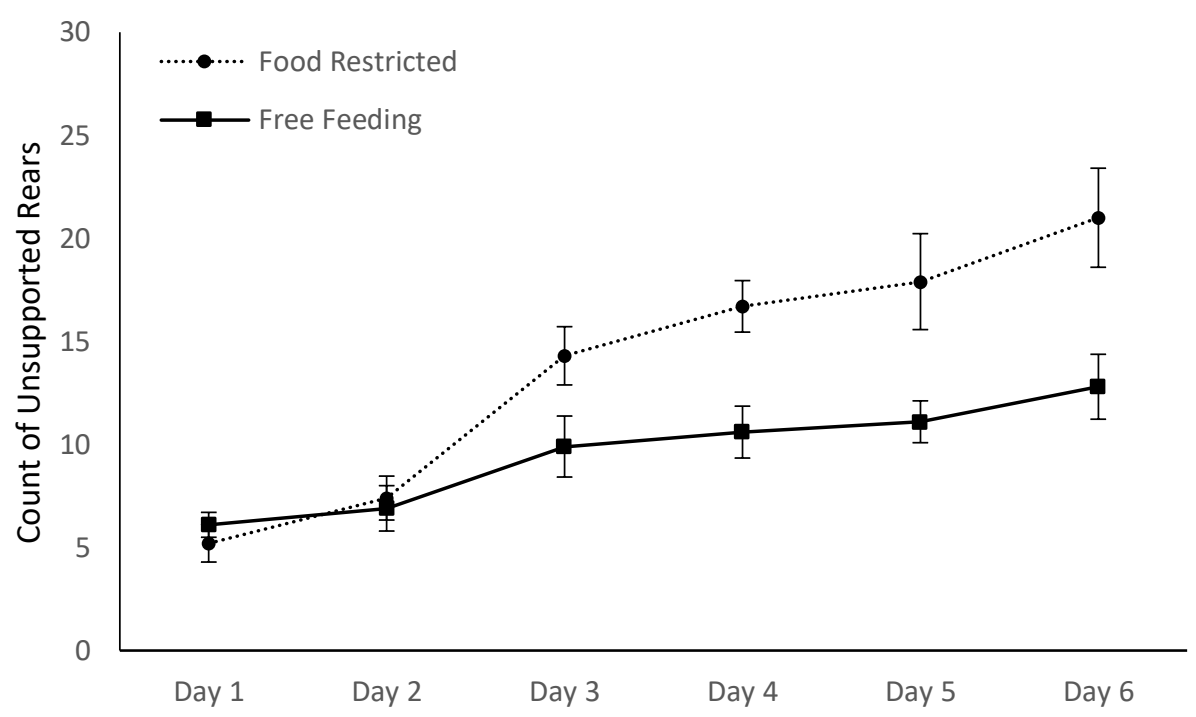

Figure 5. Means and \pm SEM of the number of unsupported rears made in the anticipation box on each day of testing. Consecutive days are presented on the $x$ axis, and the count of unsupported rears is depicted through the $y$ axis. 


\section{Latency to Alley 4}

Mauchly's test of sphericity was violated and a Greenhouse-Geisser correction was consequently used. There was a significant main effect of day $[F(5,51.22)=6.35, p<.001]$, whereby latency to enter alley 4 was significantly reduced across the days of testing (see figure 6). However, no significant main effect of condition or interaction between factors was found.

Figure 6. Latency to Enter Alley 4

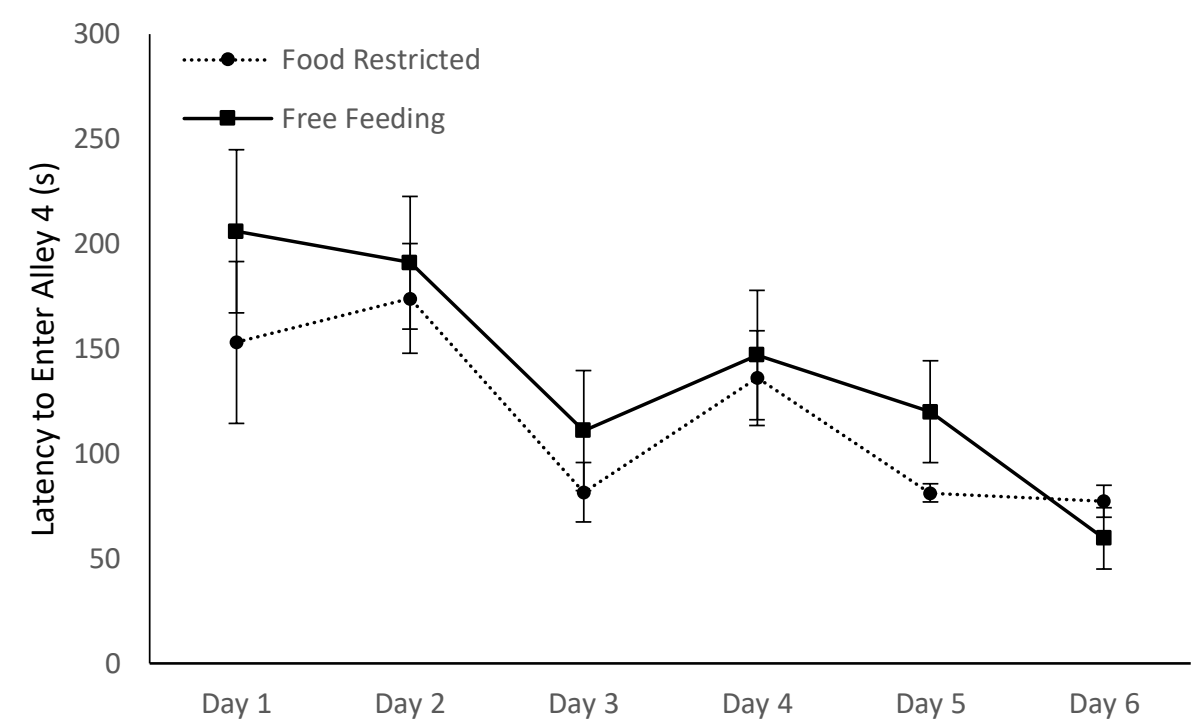

Figure 6. Means and \pm SEM of the number of the latency to enter alley 4 on each day of testing (Total trial duration 300s). Consecutive days are presented on the $x$ axis, and latency to enter in seconds is depicted through the $y$ axis.

\section{Duration in Alley 4}

A main effect of day was found $[F(5,85)=10.08, p<.001]$, with subjects spending significantly more time in alley 4 the more times they were exposed to the test (see figure 7 ). No significant main effect of condition, or significant interaction between factors was found.

\section{Retrieval Latency}

Mauchly's test of sphericity was violated, hence a Greenhouse-Geisser correction was used for the following analyses. Analysis of retrieval latencies revealed a significant main effect of day $[F(2.71,48.69)=11.55, p<.001]$, and a significant main effect of condition $[F$ 
$(1,18)=24.4, p<.001]$, with retrieval latency decreasing across the days, and the food restricted condition having fast latencies overall (see figure 8). No significant interaction was observed.

Figure 7. Cumulative Duration in Alley 4

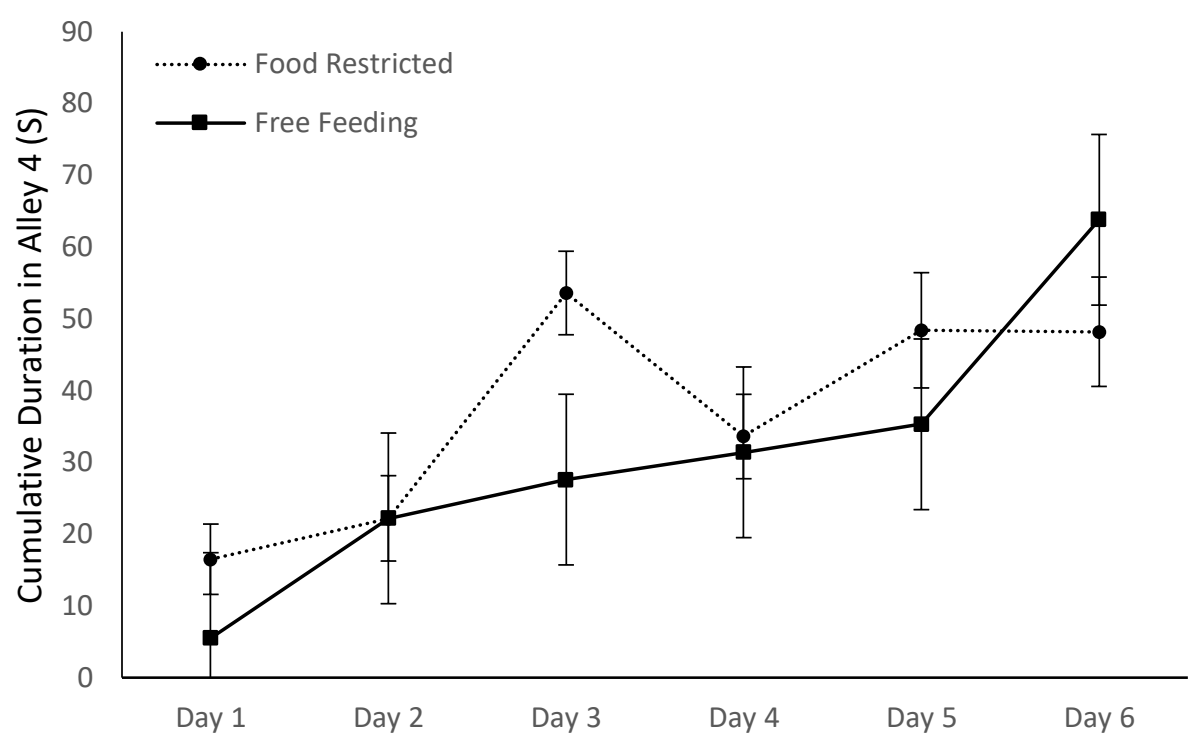

Figure 7. Means and \pm SEM of the cumulative duration spent in alley 4 on each day of testing (Total trial duration 300s). Consecutive days are presented on the $x$ axis, and duration is depicted through the $y$ axis.

Figure 8. Retrieval Latency

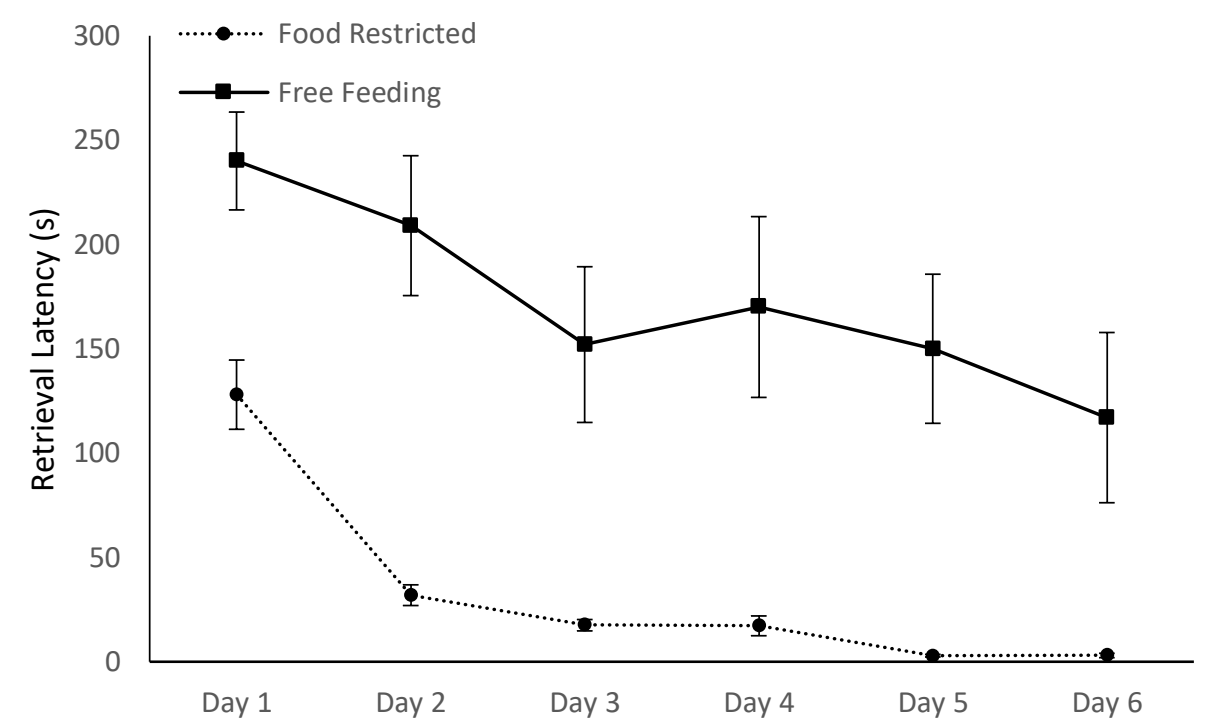

Figure 8. Means and \pm SEM of the number of the latency to consume the first frootloop on each day of testing (Total trial duration 300s). Consecutive days are presented on the $x$ axis, and duration in seconds is depicted through the $y$ axis. 


\section{Retrieval Score}

Analysis of retrieval scores also revealed a significant main effect of condition $[F(1,18)$ $=28.5, p<.001]$, with food restricted subjects exhibiting higher scores overall, while no significant main effect of day or interaction was observed (see figure 9).

Figure 9. Retrieval Score

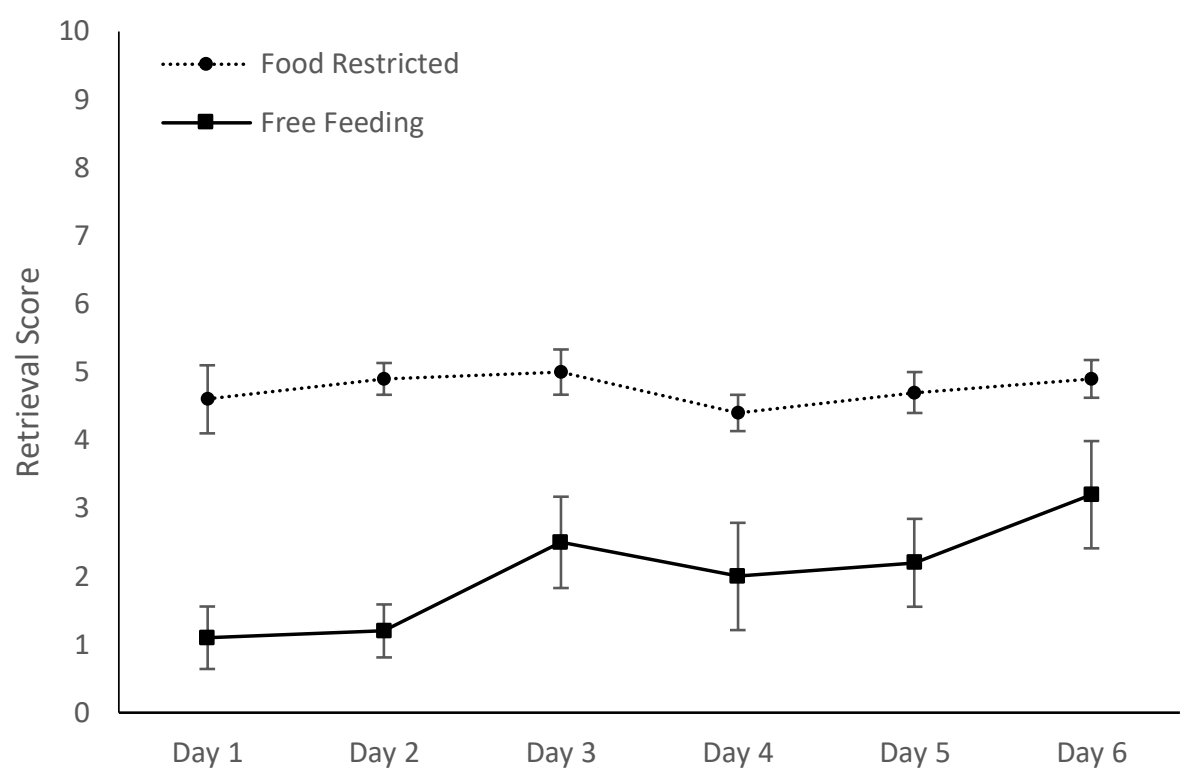

Figure 9. Means and \pm SEM of the number of retrieval scores on each day of testing. Consecutive days are presented on the $x$ axis, and score is depicted through the $y$ axis.

\section{Discussion}

This first experiment investigated whether food restriction was necessary for a) subjects to show a sufficient anticipatory response when expecting to receive a food reward, and b) subjects to repeatedly enter more anxiogenic areas of the alley.

It was hypothesized that food restricted animals would show a stronger anticipatory pleasure response compared to the free feeding condition. Results supported this hypothesis, with the food restricted condition showing a greater increase in overall rearing and $50 \mathrm{kHz}$ USVs emitted across the 6 days of testing. These findings indicate that food restriction is necessary to evoke sufficient anticipatory responses. In addition, while supported rearing increased proportionally between conditions, food-restricted subjects showed a greater increased in unsupported rearing, the measure more indicative of anticipatory pleasure, further supporting the impact of food-restriction on anticipation. 
In contrast, results showed no difference between conditions in latency to enter and duration spent in alley 4, across all 6 days of testing. This finding does not support our hypothesis that food restriction would be necessary to repeatedly entice subjects to enter more anxiogenic areas of the test. However, food-restricted subjects exhibited lower retrieval latency across all days of testing, indicating motivation to consume the bait differed between the two conditions. It is possible that food-restriction played a role in motivation to enter anxiogenic alleys initially, but with increased exposure to the test, subjects learnt there was nothing 'dangerous' on those arms and therefore did not need continuous motivation to enter them. It is important here to consider that the subjects of this experiment were Sprague-Dawley's, an essentially 'normal' phenotype. As the ADT is a tool to measure anxiety-like behaviour in rodents, future use of this test will likely employ subjects with an anxious-phenotype. Accordingly, subjects more inherently averse to the anxiogenic areas of the alley may need this additional motivation to repeatedly enter them.

Using the novel measure of food retrieval score, significant differences were found in anxiety-like behaviour between conditions. Food restriction significantly increased retrieval scores, with scores sitting roughly in the middle of the maximum number of points possible, whereas the free feeding condition showed scores on the lower end of the scale. As noted above, the subjects tested were not predisposed to be anxious, however still showed low retrieval scores when free feeding. Therefore, if employing an anxious animal model in future, it is crucial to consider that not restricting subjects may lead to a floor effect on retrieval scores. Previous research using the ADT suggested that retrieval score is the most sensitive measure of anxiety-like behaviour (Kidwell, 2019). Indexes of anxiety-like behaviour such as latency to enter and duration spent in alley 4 , only displayed differences between SERT $\%$ (high anxiety model) and SERT ${ }^{+}++(\mathrm{WT})$ groups, whereas retrieval score distinguished between $\quad \mathrm{SERT}^{+/}$ (moderate anxiety model) and SERT ${ }^{+} /^{+}$. The previous finding that retrieval score is a more sensitive measure of anxiety-like behaviour, coupled with the current studies results, suggest food-restriction is necessary to avoid potential floor effects, and to be able to capture a full range of scores.

The impact of food restriction on depression and anxiety-like behaviour is inconsistent across literature, therefore the restriction protocol used is crucial to consider. Jahng et al. (2007) investigated the impact of 5 weeks of food restriction, from PND 28 on male Sprague-Dawley rats. Subjects showed increased immobility time in the FST and decreased duration spent in open arms of the EMP, indicating increased depressive and anxiety-like behaviour, compared to free feeding controls. Similarly, Iio and colleagues (2015) report that 5 weeks of food 
restriction from PND 56, increased immobility time in the FST in male Wistar rats. However, these authors also report the same restriction protocol decreased anxiety-like behaviour in the open field test. These conflicting findings may be due to differences in the subject's strain, age, or the test paradigms used.

The current study employed an acute food restriction regime, with subjects being restricted for 11 days total. As the ADT was designed with the intention of eliminating the onetrial-tolerance, future research may employ this test to investigate longer lasting symptoms of disorders, multiple treatments in the same animal, duration to onset of efficacy, and duration to remission after ceasing treatment. These future directions will involve repeatedly testing subjects over an extended period of time, therefore research with these aims must consider the impact of a chronic food restriction protocol on depressive and anxiety-like behaviour.

In addition, the finding that food restriction increases corticosterone levels (Heiderstadt et al., 1999; Marinelli et al., 1996; Toth \& Gardiner, 2000) warrants consideration in terms of the moderating effects this may have on behaviour and drug sensitization.

Various studies have shown corticosterone administration selectively decreased immobility in the FST in female but not male rats (Brotto et al., 2001; Hill et al., 2003), whereas others have indicated corticosterone increases immobility in male rats (Koike et al., 2013; Ulloa et al., 2010). Both cases indicate the effects of corticosterone are sex specific, and may have a paradoxical impact on depression-like behaviour that needs to be considered when food restricting subjects in the ADT.

Corticosterone levels have also been implicated in behavioural sensitization to drugs of abuse. Marinelli and colleagues (1996) found that subjects that were food restricted to $90 \%$ of their free feeding weight, followed by 30 minutes of restraint stress, showed higher levels of corticosterone and increased behavioural sensitivity to cocaine compared to ad libitum subjects exposed to the same restraint stress. Treatment with metyrapone (a corticosterone synthesis inhibitor) abolished these effects, supporting the conclusion that the sensitization to cocaine produced by stress is moderated by an increase in corticosterone levels due to food restriction. In line with previous findings, Cadoni et al. (2003) report that rats food restricted to $80 \%$ of initial body weight for 1 week showed behavioural sensitization to cocaine and amphetamine compared to ad libitum controls. This sensitization was not found for morphine or nicotine. These findings suggest that increased corticosterone release due to the stress induced via food restriction may play a role in drug intake. Numerous studies have recognized a high level of comorbidity between substance abuse and mood disorders such as major depression and anxiety (Aseltine et al., 1998; Neighbors et al., 1992), presenting a direction for future research 
with the ADT. However, as food restriction increases corticosterone levels, which in turn differently impacts behavioural sensitization to some drugs of abuse, these effects must be considered if assessing the impact of drugs of abuse in ADT.

Conversely, Soubrié et al. (1988) demonstrated that restricting subjects to $85-90 \%$ of their free feeding weight has been found to attenuate the efficacy of antidepressants in a learned helplessness paradigm. Furthermore, this effect was observed across a variety of treatment classes (monoamine oxidase inhibitors, norepinephrine reuptake inhibitors, and serotonin reuptake inhibitors). Similarly, restriction to $92 \%$ of free feeding weight diminished the antidepressant effects of escitalopram in the FST (France et al., 2009). The attenuation of antidepressant effects with food restriction may confound ability to detect treatment efficacy, therefore requires further investigation into the direct impact this may have on the predictive validity of the ADT.

The current study has implications for establishing a protocol for future use of the ADT. Subjects were habituated to the anticipation box for 5 days in order to reduce novelty-induced exploration confounding anticipation responses in testing. Our results showed a decrease in rearing from day 1 to 3 of habituation, however behaviour remained stable from day 3 onwards (data not shown). Based off this finding, it is suggested that only 3 days of habituation to the anticipatory box is necessary when using the ADT in future research.

A central aim of this thesis is to assess the ADTs sensitivity to pharmacological treatment. If using an acute antidepressant treatment, the onset of a typical anticipatory pleasure response must be considered. In order to assess a treatment's impact on anticipatory pleasure responses, subjects need to have associated the anticipation box with receiving frootloops. The current study showed an increase in rearing on day 3 and in $50 \mathrm{kHz}$ calls on day 4 compared to previous days (analysis not reported), indicating an anticipatory pleasure response. Therefore, we recommend an acute antidepressant treatment should be given at least 5 days into testing.

The findings of this first experiment endorse the incorporation of food restriction into the ADT protocol. Moreover, these results substantiate the use of the ADT as a tool to assess depressive and anxiety-like behaviours in rats. In line with previous research which employed this test (Kidwell, 2019; O'Connell, 2020), findings indicate the ADT is not subject to the onetrial tolerance found in other tests of anxiety, as subjects continued to enter anxiogenic alleys after initial exposure. Furthermore, subjects showed anticipatory pleasure responses in the anticipation box, supporting the test's ability to measure anhedonia, a more valid index of depression-like behaviour than 'behavioural despair' measured in tests such as the forced swim and tail suspension tests. 


\section{Chapter Three}

\section{Evaluating the Construct and Predictive Validity of the}

\section{ADT}


Well established tests of depression and anxiety-like behaviour in rats such as the forced swim test and elevated plus maze are widely used due to their predictive validity, whereby effective pharmacological treatment in humans also improves behaviour in these tests. Additionally, the elevated plus maze displays construct validity, as animal models with theoretical relevance to the human condition of these disorders exhibit corresponding anxietylike behaviour. For example, rodents exposed to environmental factors such as chronic stress (Monteiro et al., 2015) and maternal separation (Jin et al., 2018), as well as genetic manipulations to disrupt GABA (Tasan et al., 2012) and serotonin systems (Smits et al., 2006), display increased anxiety-like behaviour in this test, paralleling findings in the human population.

Establishing the construct and predictive validity of a test is essential to ensure the translatability of the animal model to the human condition. A test which possesses this validity constitutes a valuable tool for understanding the foundation of such disorders and subsequently the development of treatments.

To further support the use of the ADT as a novel measure of depression and anxietylike behaviour in rats, this second experiment investigates the construct and predictive validity of this test by assessing the behaviour of an animal model of depression and anxiety, and the impact of pharmacological treatment.

\section{Construct Validity}

It is well established that serotonin (5-HT) systems play a large role in mood disorders, with disturbances to this system being heavily implicated in the onset of depression and anxiety (Serretti et al., 2007). The serotonin transporter (SERT) is the primary regulator of 5-HT in the brain. Its role is to reuptake 5-HT from the extracellular space into the presynaptic neuron where it can be released again. In humans, SERT transcription is modulated by a common polymorphism (5-HTTLPR) in the SLC6A4 gene. A 44 base pair insertion/deletion leads to a long allelic (1) and short allelic (s) variant respectively. Transcriptional efficiency of the 5-HTT gene is reduced in humans with the short variant of the polymorphism, and those with an ssgenotype show a 50\% reduction in SERT activity compared to the ll-genotype (Nakamura et al., 2000; Oliver et al., 2008). Furthermore, there is a well-established link between the short variant of this polymorphism and psychiatric conditions, including mood and anxiety disorders (Olivier et al., 2008). 
Genetic manipulation techniques such as N-ethyl-N-nitrosourea (ENU mutagenesis) have allowed researchers to generate a rodent model with reduced 5-HTT expression, coined the SERT knockout (Slc6a4 ${ }^{\text {Hubr }}$ ) rat (Smits et al., 2006). Follow up research on this model showed a complete absence of SERT in the homozygous knockout (SERT \%), and a 50\% reduction of SERT levels in the heterozygous knockout (SERT ${ }^{+}$) compared with wildtypes $\left(\right.$ SERT $\left.^{+} /^{+}\right)$. Additionally, basal levels of 5 -HT were found to be dramatically reduced in the SERT $\%$, while other monoamine systems remained unaltered (Homberg et al., 2007).

The SERT knockout rats have subsequently tested on a variety of behavioural assays. Numerous studies have found that the SERT $\%$ strain exhibits consistent anxiety-like symptoms on the elevated plus maze and open field test (Mohammad et al., 2016; Olivier et al., 2008). In line with human neurology associated with anxiety disorders, Jiang and colleagues (2009) demonstrated impairments in HPA axis in SERT knockout mice, further validating this strain as a model for anxiety disorders. Additionally, this strain shows reduced sucrose preference and increased immobility in the FST compared to the SERT ${ }^{+} /^{+}$strain, indicating depressivelike symptoms (Olivier et al., 2008). In sum, the SERT knockout rat has displayed widespread applicability in modelling mood disorders, providing a valuable translational model of major depression and anxiety disorder.

As the SERT knockout rat exhibits depression and anxiety-like behaviours in established tests, the current study will employ the SERT knockout rat as a model of depression and anxiety to assess the construct validity of the ADT. The SERT model is favourable as it has relevance to the variants found of the SERT gene found in humans, compared to other animal models which are selectively bred for their specific depressive/anxious traits, such as the Flinders sensitive line (Overstreet, 1993), and High/Low Anxiety Related Behaviour model (Leibsch et al., 1998). The ADT has been created to provide a test for affective disorders which is more relevant to the human condition, therefore using a translational animal model is crucial. The SERT model will allow us to assess both depression and anxiety components of the test in the same animal, which is beneficial considering the time restraints of this thesis.

\section{Predictive Validity}

A key asset of the traditional tests is their predictive validity. The rapid increase in the prevalence of affective disorders, coupled with the growing awareness of undesirable sideeffects of many antidepressant and anxiolytic drugs, raises the need for tests which can screen pharmacological treatments effectively. 
Diazepam is one of the most commonly used drugs in the treatment of anxiety in the human population (Pringle et al., 2016). In addition, it shows consistent anxiolytic effects in established animal tests of anxiety-like behaviour after acute administration, making it the gold standard for evaluating predictive validity in animal models of anxiety.

In both male and female Long Evans rats, a single dose of 1-3 mg/kg of diazepam (i.p), reduced anxiety-like behaviour in the EPM in a dose-dependent manor (Wilson et al., 2004). The authors did not find any differences in sensitivity to diazepam between sexes, or any impact on locomotor activity at all treatment doses. Similarly, 1 and $1.5 \mathrm{mg} / \mathrm{kg}$ increased the duration spent in the open arms of the maze in Dark Agouti and Sprague-Dawley rats. However, treatment significantly decreased the total number of arm entries in Dark Agouti, but not Sprague-Dawley subjects, indicating diazepam may have strain a dependent impact on locomotor activity (Mechan et al., 2002). Rex et al. (1996) assessed a range of different doses $(0.3,0.6,1.2,2.5,5 \mathrm{mg} / \mathrm{kg}$, i.p.) in male Wistar rats in the EPM. The number of entries into the open arms increased in a dose-dependent manner from the dose of $0.6 \mathrm{mg} / \mathrm{kg}$, whilst time spent in the open arm increased in a dose-dependent manner from the dose of $1.25 \mathrm{mg} / \mathrm{kg}$, indicating dose sensitivity between different measures of anxiety-like behaviour. Furthermore, the reduction in anxiety-like behaviour in the EPM following a single administration of $5 \mathrm{mg} / \mathrm{kg}$ (i.p.), 30 minutes prior to testing, was analogous to the reduction following chronic (14 day) treatment, demonstrating no differences in efficacy between acute and chronic treatment (Wright et al., 1991). Diazepam has also demonstrated efficacy across a range of tests such as the light/dark box (Chaouloff \& Mormede, 1997), novelty suppressed feeding test (Bodnoff et al., 1988), and open field test (Bahi et al., 2014), at doses of $1-3 \mathrm{mg} / \mathrm{kg}$. When investigating behaviour on the High-Anxiety Related-Behaviour (HAB) and Low-Anxiety-RelatedBehaviour (LAB) Wistar rat lines, the HAB line consistently shows greater anxiety-like behaviour in the elevated plus maze compared to the LAB (Henniger et al., 2000; Leibsch et al.,1998). Diazepam (1 mg/kg, i.p) administration results in anxiolytic effects in both lines, however it had a significantly greater effect in the HAB line, indicating this animal model of anxiety is more sensitive to the anxiolytic properties of diazepam (Leibsch et al.,1998).

Overall, previous literature endorses the use of diazepam as a benchmark treatment for eliciting anxiolytic effects in different models. However, research also suggests that doses above $3 \mathrm{mg} / \mathrm{kg}$ may produce motor impairments (File \& Fernandes, 1994); Kudagi et al., 2012), which would interfere with the interpretation of data. For that reason, we piloted a dose of $2 \mathrm{mg} / \mathrm{kg}$, which resulted in a significant decrease in locomotor activity (data not shown), therefore a dose of $1 \mathrm{mg} / \mathrm{kg}$ was selected for the current experiment. 
As the ADT aims to measure both anxiety and depressive-like behaviours, the test's sensitivity to an antidepressant treatment must also be assessed. The FST has become one of the most widely used tests of depressive-symptoms based off its predictive validity (Slattery \& Cryan, 2012). All major classes of antidepressants including tricyclic, selective serotoninreuptake inhibitors, and monoamine oxidase inhibitors, have been found to reduce immobility time on this test (Detke et al., 1997). Willner (1984) reported a significant correlation between clinically effective human doses and the behavioural effects seen in the FST, further supporting this tests ability to screen antidepressant drugs, and assess effective doses. Furthermore, most other psychoactive substances including antipsychotics and anxiolytic drugs fail to elicit behavioural changes, indicating the FST is adequately selective for antidepressants (Detke et al., 1997; Porsolt et al., 1997).

An intricacy of assessing a test's sensitivity for antidepressant treatment is that paralleling clinical studies, the effects of most antidepressants are also only seen after chronic treatment. Detke and colleagues (1997) found no effect of treatment on behaviour in the FST with antidepressants desipramine and fluoxetine when administered twice (23.5 and 1 hour before testing), however did find significant improvements to depressive-like behaviour when the same drugs and dose were administered chronically (once daily for 14 days prior to testing). Similarly, Cryan et al. (2005) found no effects of short term (once daily for 3 days) treatment of reboxetine and fluoxetine, whereas the same drugs and dose were effective after 14 days of treatment.

Due to the scope of the current project, having the ability to use the same subjects as a control group for both treatment conditions was desirable. As diazepam and the subsequent vehicle were administered acutely, and due to the utilization of the SERT knockout model, we were required to use a treatment that works acutely, and does not directly affect the serotonergic system.

A growing body of literature recognises the N-methyl-D-aspartate (NMDA) glutamate receptor as a key player in mood disorders such as major depression (Sanacora et al., 2008). Studies have found several NMDA receptor antagonists have antidepressant effects across multiple animal models of depression, such as the FST, tail suspension test, and sucrose preference test (Zarate et al., 2006). Ketamine hydrochloride is a potent NMDA antagonist, and shows promising antidepressant effects in human clinical trials, after just a single treatment (Zarate et al., 2006). Antidepressant effects after acute administration have also been demonstrated in animal research. Garcia and colleagues (2008) demonstrated decreased immobility time of adult, male Wistar rats in the FST 60 minutes after ketamine treatment 
$(10 \mathrm{mg} / \mathrm{kg}$ and $20 \mathrm{mg} / \mathrm{kg}$, i.p.). However, this antidepressant effect was not found at a dose of $5 \mathrm{mg} / \mathrm{kg}$. As ketamine has anaesthetic effects, it is crucial to assess its impact on locomotor activity. At all doses tested, the authors found no impact to locomotor activity, indexed by behaviour in the open field test. Similar results were obtained by Assis and colleagues (2009), supporting the antidepressant ability of ketamine at a dose of $10 \mathrm{mg} / \mathrm{kg}$.

In contrast to the findings of Garcia et al., (2008), further research indicated that when administered 30 minutes prior to testing, a dose of $5 \mathrm{mg} / \mathrm{kg}$ decreased immobility time in male Wistar and Sprague-Dawley rats (Carrier \& Kabbai, 2013; Yang et al., 2013). Furthermore, 2.5 $\mathrm{mg} / \mathrm{kg}$ produced anti-depressant effects in the FST and novelty suppressed feeding test in female, but not male, Sprague-Dawley rats (Carrier \& Kabbai, 2013).

Using spared nerve injury (SNI) as a tool to induce depression-like behaviour, Wang and colleagues (2011) investigated the impact of 1,3,10 and $20 \mathrm{mg} / \mathrm{kg}$ (i.p) 24 hours prior to testing on behaviour in the sucrose preference test. Untreated, SNI produced an increase in depression-like behaviour, with doses of 10 and $20 \mathrm{mg} / \mathrm{kg}$ resulting in antidepressant effects. After establishing the antidepressant effects of a $10 \mathrm{mg} / \mathrm{kg}$ dose, authors next assessed the impact of this dose on behaviour in the FST. Immobility time of the SNI ketamine treated group was comparable to that of the sham condition, indicating $10 \mathrm{mg} / \mathrm{kg}$ normalised behaviour of 'depressed' subjects.

The above findings outline the antidepressant effects of ketamine on established tests, and therefore support the use of this treatment in evaluating the predictive validity of the ADT. A lower dose of $5 \mathrm{mg} / \mathrm{kg}$ was selected with the intention of reducing the possibility of a higher dose concealing genotype differences in efficacy.

To assess the construct validity of the ADT, the behaviour of SERT ${ }^{++}(\mathrm{WT})$ and SERT $\%$ rats was investigated across 4 drug free days. Given the findings that SERT $\%$ rats display depression and anxiety-like behaviour in established tests (Olivier et al., 2008), it was hypothesised that SERT $\%$ subjects would display increased depression and anxiety-like behaviour in the ADT compared to WT subjects. Thus, we predicted SERT \% would show a decrease in rearing and 50kHz USVs in the anticipation box. Likewise, we predicted SERT $\%$ will spend a lower duration in anxiogenic alleys, have a greater latency to enter these alleys, and display lower retrieval score in the SAT.

To investigate the predictive validity of the ADT, subjects were administered either $1 \mathrm{mg} / \mathrm{kg}$ diazepam, $5 \mathrm{mg} / \mathrm{kg}$ ketamine, or saline on day 5 of testing. Day 5 was selected based off results from experiment one, which indicated subjects showed anticipatory pleasure at day 3 when indexed by rearing, and 4 indexed by USVs. As numerous studies have demonstrated 
the antidepressant and anxiolytic effects of ketamine and diazepam, respectively, we hypothesise each treatment will improve behaviour on the relevant aspects of the test. Hence, it was predicted that ketamine would increase rearing behaviour and $50 \mathrm{kHz}$ USVs, whereas diazepam would increase the duration spent in anxiogenic alleys, decrease latency to enter these alleys, and lastly, increase retrieval scores.

Furthermore, we hypothesised a gene-treatment interaction, whereby treatment would have a greater effect in the SERT $\%$ group compared to the WT rats.

\section{Method}

\section{Subjects}

A separate cohort of 49 male Wistar rats were used for experiment two. Two different genotypes were used: 24 homozygous SERT knockout (SERT ${ }^{-}$) and 25 Wildtype animals lacking the SERT knockout mutation $\left(\mathrm{SERT}^{+} /^{+}\right.$). All subjects were of adult age (PND 60 -85). Based off the results of the previous experiment, animals were maintained at $85-90 \%$ of their free feeding weight for one week prior to testing. This weight was maintained using the protocol outlined for the food restricted condition of experiment one. All animals were bred and housed in the same conditions as experiment one and procedures were approved under the same AEC (27328).

\section{Pharmacological treatment}

Subjects were exposed to a single intraperitoneal (i.p.) injection of either $5 \mathrm{mg} / \mathrm{kg}$ of ketamine hydrochloride (supplied by Pheonix Pharm Distributors Ltd., Auckland, New Zealand, $12.5 \mathrm{mg} / \mathrm{mL}$ ), $1 \mathrm{mg} / \mathrm{kg}$ of diazepam (Ilium, $5 \mathrm{mg} / \mathrm{mL}$ ), or saline. Table 1 outlines the number of subjects per genotype to receive each treatment.

Ketamine and saline were administered at a volume of $0.4 \mathrm{ml} / \mathrm{kg}$, and diazepam was administered at a volume of $0.2 \mathrm{ml} / \mathrm{kg}$. Diazepam was initially administered to $2 \mathrm{WT}$ subjects at a volume of $0.4 \mathrm{ml} / \mathrm{kg}$, however this dose of $2 \mathrm{mg} / \mathrm{kg}$ severely impacted locomotor activity. Therefore administration volume was reduced to $0.2 \mathrm{ml} / \mathrm{kg}$ as a means of reducing dose to 1 $\mathrm{mg} / \mathrm{kg}$. All treatments were administered 45 minutes prior to testing (35 mins prior to habituation to experimental room). 
Table 1. $n$ per condition

\begin{tabular}{llll}
\hline Genotype & Ketamine & Diazepam & Saline \\
\hline SERT $\%$ & $n=7$ & $n=9$ & $n=8$ \\
WT & $n=8$ & $n=10$ & $n=7$
\end{tabular}

Note. The 2 WT subjects that were administered the higher $(2 \mathrm{mg} / \mathrm{kg})$ diazepam dose were removed from data analysis.

\section{Experimental Setting and Procedure}

The experimental setting was identical to that of experiment one.

Animals were habituated to the anticipation box following the same procedure as experiment one. However, the habituation period was decreased to 3 days, as the previous experiment indicted rearing behaviour had stabilised after 2 days of habituation. In line with the previously established habituation protocol, animals were also handled and exposed to frootloops in their home cage over the course of these 3 days.

The first 4 days of testing followed the same procedure as experiment one. On day 5 of testing, subjects were exposed to the relevant pharmacological treatment 45 minutes prior to the test. From day 6 onwards, animals were tested for an additional 4 days (drug free) to investigate whether the behaviour would return to baseline.

\section{Statistical Analysis}

Experiment one indicated that 4 days of testing is sufficient to detect anticipatory pleasure responses, and for anxiety-like behaviour to have stabilized. Therefore, as subjects were untreated on days $1-4$, these first 4 days of testing were used to investigate genotype differences in behaviours in the ADT. To assess these differences, a repeated measures mixed ANOVA was run for each measure, with day (1-4) as a within-subjects variable, and genotype (WT, SERT $\%$ ) as a between-subjects variable.

To assess the predictive validity of the ADT, the acute impact of diazepam and ketamine on each measure was analyzed using a mixed factorial ANOVA, with days $(4,5)$ as the repeated measure, and treatment (saline, treatment) and genotype (WT, SERT ${ }^{-}$) as between groups factors. Because the treatments are not comparable to each other, each treatment was analyzed compared only to the control condition. Day 4 was included in this 
analysis as it allowed us to investigate the impact of treatment compared to previous behaviour and ensure that differences seen at day 5 were due to treatment effects, not differences existing within the genotype prior to treatment.

To investigate whether behaviour returned to baseline after drug administration, the long-term impact of treatment was investigated. A second mixed factorial ANOVA was conducted, with days (5-9) as the repeated measure, and treatment (saline, treatment) and genotype (WT, $\mathrm{SERT}^{-}{ }^{-}$) as between groups factors.

Following each analysis, appropriate pairwise comparisons of each treatment were run and the Bonferroni correction applied to adjust for multiple comparisons. As the number of pairwise comparisons made depends on which interactions were statistically significant, the adjustments made varied between analyses and therefore adjusted p-values are reported alongside the presentation of results. For example, following significant day*genotype*treatment interactions, comparisons of each treatment on each genotype at each day were run, but following significant genotype*treatment interactions, only comparisons of each treatment on each genotype were run.

\section{Results}

All results from each set of the analyses mentioned above are presented in tables $2-$ 10. Additionally, descriptive statistics are presented in appendix A - I.

\section{Anticipation Box}

USVS

To account for large individual differences in the total number of USVs made, USVs were assessed as the proportion of $50 \mathrm{kHz}$ calls made out of the total calls on each day. 16 trials were removed from USV analysis, 7 due to file corruption, and 9 due to subjects making no calls, meaning we were unable to calculate a proportion of $50 \mathrm{kHz}$ calls.

When assessing genotype differences on days $1-4$, results showed a main effect of day, where the proportion of $50 \mathrm{kHz}$ calls made increased across the days (see figure 10). No genotype effect, or day*genotype interaction was found (see table 2). 
Figure 10. Genotype Differences in Proportion of 50kHz USVs

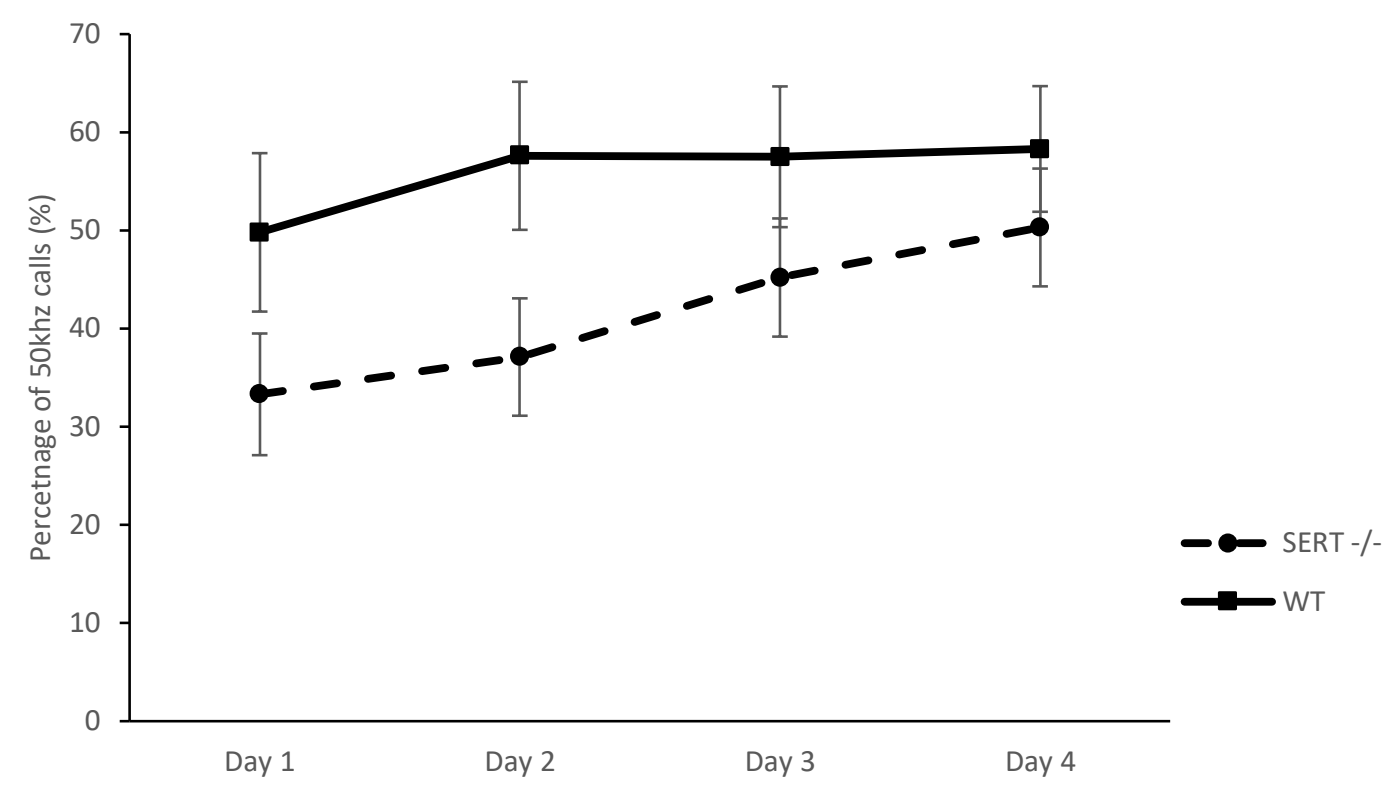

Figure 10. Means and \pm SEM of genotype differences in the percentage of $50 \mathrm{kHz}$ calls made on the first four days of testing. Consecutive days are presented on the $x$ axis, and percentage of calls is depicted through the $y$ axis.

When assessing the acute impact of diazepam on $50 \mathrm{kHz}$ calls, results showed a main effect of day, and day*treatment interaction. Follow up planned comparisons indicated no significant difference between treatment groups on both day 4 and day 5 . However, based off the findings depicted in Figure 11, further investigation into the differences between days for each condition was conducted. These comparisons revealed that in WT subjects, diazepam treatment significantly reduced the proportion of calls made on day 5 when compared to the WT saline $(\operatorname{adj} p<.001)$ and WT diazepam (adj $p=.028)$ groups on day 4, whereas WT subjects treated with saline on day 5 did not differ when compared to both WT groups on day 4. Additionally, no differences were found between SERT $\%$ treatment groups across these days.

The long-term impact of diazepam also showed a significant day*treatment interaction, however planned comparisons also indicated no significant difference between treatment groups across days $5-9$. As above, this interaction appears to be driven by the decrease in proportion of $50 \mathrm{kHz}$ calls made by the diazepam WT group on day 5 (see figure 12).

Ketamine had no effect on the proportion of $50 \mathrm{kHz}$ calls made, both acutely and longterm (see table 2 \& appendix $A$ ). 
Figure 11. Acute Impact of Diazepam on Proportion of 50kHz USVs

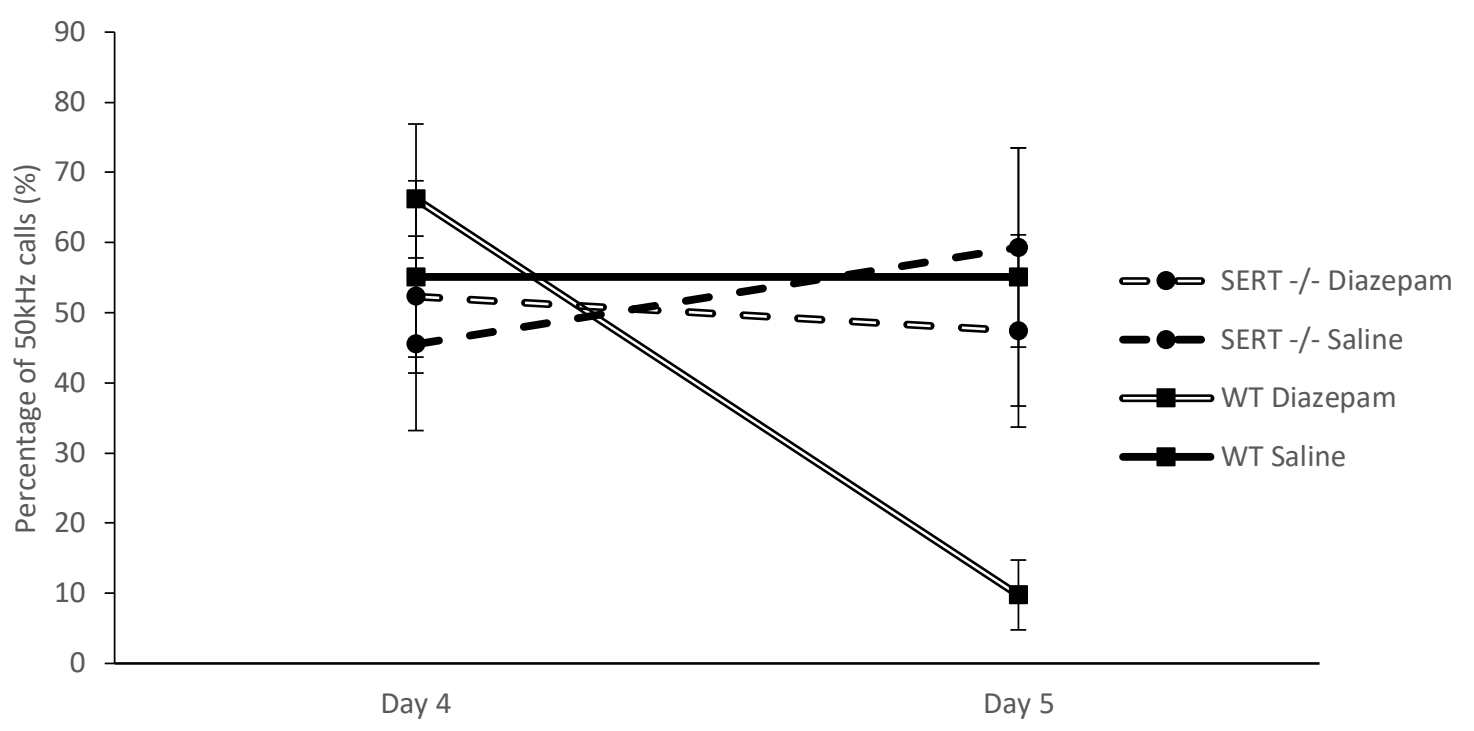

Figure 11. Means and \pm SEM of the acute impact of diazepam on percentage of $50 \mathrm{kHz}$ calls. Consecutive days are presented on the $x$ axis, and percentage of calls is depicted through the $y$ axis.

Figure 12. Long-Term Impact of Diazepam on Proportion of 50kHz USVs

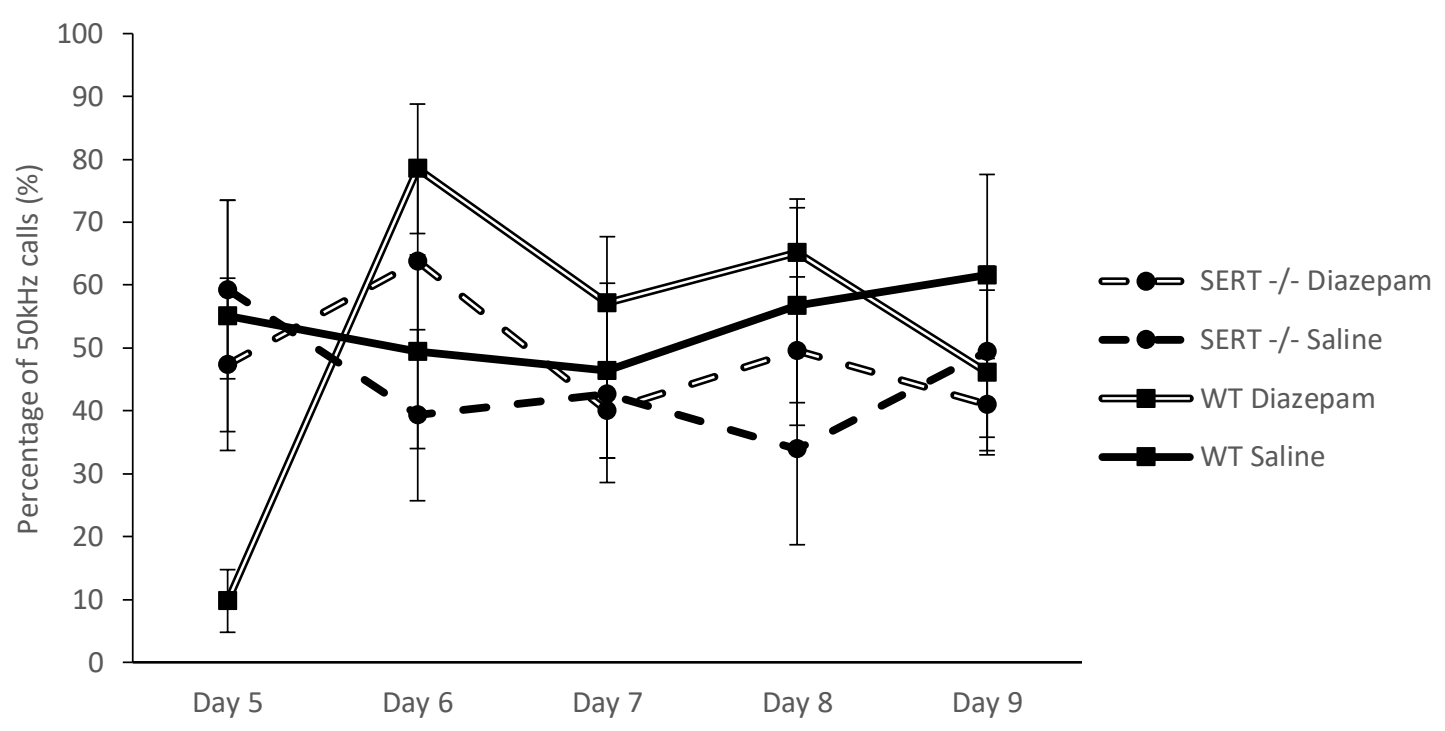

Figure 12. Means and \pm SEM of the long-term impact of diazepam on percentage of $50 \mathrm{kHz}$ calls. Consecutive days are presented on the $x$ axis, and percentage of calls is depicted through the $y$ axis. 
Table 2. Summary of analyses on Proportion of 50kHz USVs

\begin{tabular}{|c|c|c|c|}
\hline Analysis & Model Predictors & F Statistic & p-value \\
\hline \multirow[t]{3}{*}{ Genotype Differences } & Day & $F(3,117)=3.57$ & $.016 *$ \\
\hline & Genotype & $F(1,39)=3.05$ & .088 \\
\hline & Day*Genotype & $F(3,117)=1.17$ & .324 \\
\hline \multirow[t]{7}{*}{ Diazepam Acute } & Day & $F(1,20)=8.09$ & $.010^{*}$ \\
\hline & Genotype & $F(1,20)=0.08$ & .779 \\
\hline & Treatment & $F(1,20)=0.19$ & .734 \\
\hline & Day*Genotype & $F(1,20)=6.87$ & $.016^{*}$ \\
\hline & Day*Treatment & $F(1,20)=11.12$ & $.003 * *$ \\
\hline & Genotype*Treatment & $F(1,20)=0.67$ & .422 \\
\hline & Day*Genotype*Treatment & $F(1,20)=1.32$ & .259 \\
\hline \multirow[t]{7}{*}{ Diazepam Long-Term } & Day & $F(4,76)=2.38$ & .058 \\
\hline & Genotype & $F(1,19)=0.63$ & .436 \\
\hline & Treatment & $F(1,19)=0.00$ & .973 \\
\hline & Day*Genotype & $F(4,76)=2.03$ & .098 \\
\hline & Day*Treatment & $F(1,76)=5.75$ & $<.001 * * *$ \\
\hline & Genotype*Treatment & $F(1,19)=0.04$ & .853 \\
\hline & Day*Genotype*Treatment & $F(4,76)=1.48$ & .216 \\
\hline \multirow[t]{7}{*}{ Ketamine Acute } & Day & $F(1,24)=0.01$ & .920 \\
\hline & Genotype & $F(1,24)=0.17$ & .747 \\
\hline & Treatment & $F(1,24)=0.17$ & .686 \\
\hline & Day*Genotype & $F(1,24)=0.37$ & .547 \\
\hline & Day*Treatment & $F(1,24)=0.36$ & .556 \\
\hline & Genotype*Treatment & $F(1,24)=0.05$ & .826 \\
\hline & Day*Genotype*Treatment & $F(1,24)=0.74$ & .398 \\
\hline \multirow[t]{7}{*}{ Ketamine Long-Term } & Day & $F(4,84)=1.06$ & .384 \\
\hline & Genotype & $F(1,21)=0.16$ & .693 \\
\hline & Treatment & $F(1,21)=0.20$ & .662 \\
\hline & Day*Genotype & $F(4,84)=0.36$ & .834 \\
\hline & Day*Treatment & $F(4,84)=0.99$ & .418 \\
\hline & Genotype*Treatment & $F(1,21)=0.24$ & .631 \\
\hline & Day*Genotype*Treatment & $F(4,84)=2.28$ & .069 \\
\hline
\end{tabular}

Note. $* p<.05, * * p<.01, * * * p<.001$ 


\section{Rearing Behaviour}

Experiment one investigated changes to supported and unsupported rearing separately. As the count of unsupported rears, the measure considered to be more reflective of anticipation, reflected a similar pattern of results as the total number of rears, the current experiment focused on total rearing.

Analysis of genotype differences in the total number of rears made on days 1 - 4 showed a significant main effect of day and genotype, and a day*genotype interaction (see table 3). As depicted in Figure 13, there was a greater increase in rearing behaviour in the WT compared to SERT $\%$ subjects across the days.

Figure 13. Genotype Differences in Rearing Behaviour

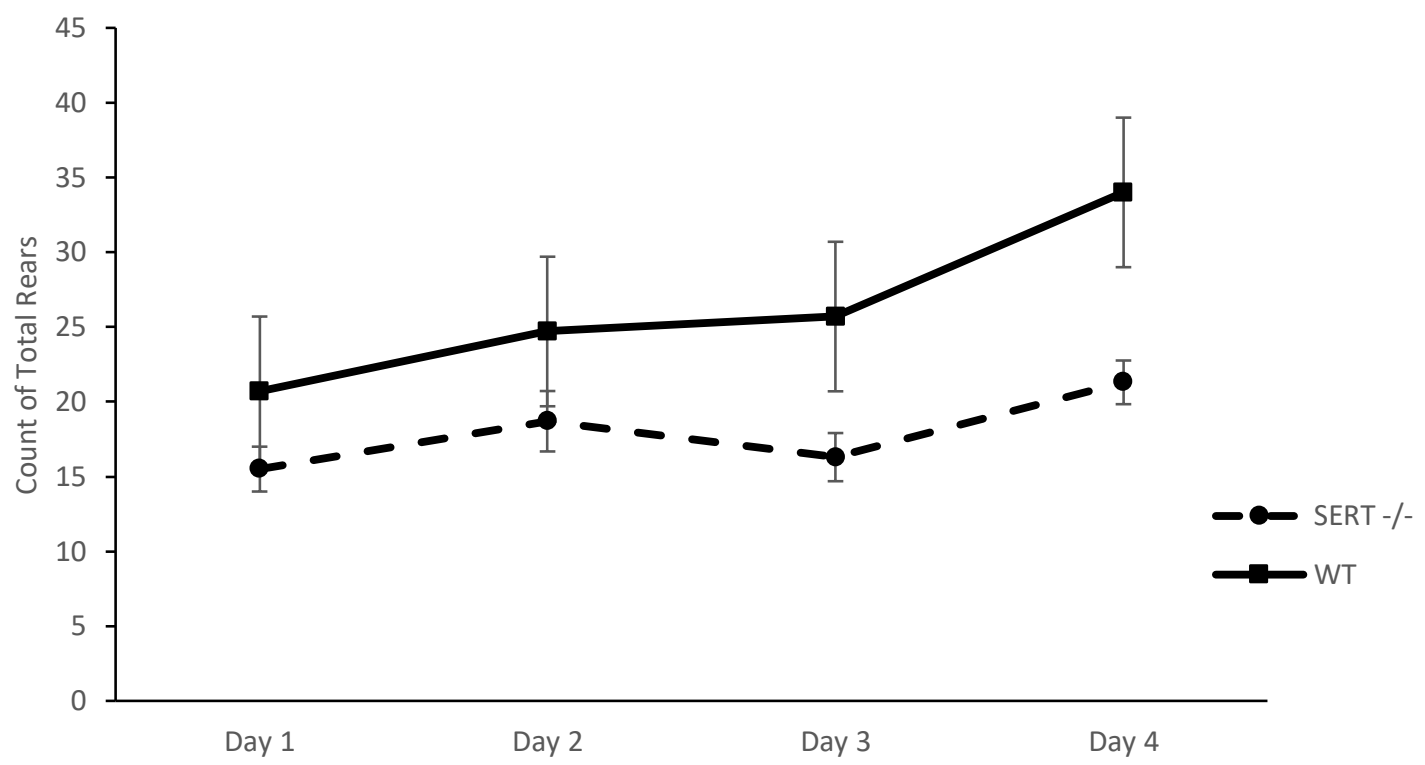

Figure 13. Means and \pm SEM of genotype differences in rearing behaviour on the first four days of testing. Consecutive days are presented on the $x$ axis, and total number of rears made is depicted through the $y$ axis.

Analysis of the acute impact of diazepam on rearing showed main effects of day, genotype and treatment. Significant day*treatment and day*genotype*treatment interactions were also found (see table 3). Follow up pairwise comparisons of the day*genotype*treatment interaction showed no difference between treatment groups within each genotype on day 4 . On day 5, the WT diazepam condition reared significantly less than the WT control condition (adj $p<.001$ ), whilst there was no difference between treatments for the $\mathrm{SERT}^{-}{ }^{-}$subjects (see figure 14). 
When investigating the long-term impact of diazepam on rearing behaviour, results showed a main effect of day, genotype and treatment. A significant day*treatment interaction was also found. Follow up analysis of the interaction indicated that on day 5 diazepam treated subjects reared less than controls (adj $p<.001)$, with no differences between treatment groups across days 6-9 (see figure 15).

The acute impact of ketamine on rearing showed a genotype*treatment and day*genotype*treatment interaction. Follow up pairwise comparisons of the impact of each treatment on each genotype at each day, showed no difference between treatment groups within each genotype on day 4. However, on day 5 ketamine treated SERT $\%$ subjects reared significantly more than controls (adj $p=.024$ ), while rearing was not significantly different between treatment groups for WT subjects (see figure 16).

When investigating the long-term effect of ketamine, a significant main effect of day and genotype, and genotype*treatment interaction was found. Following this analysis, comparisons of each treatment on each genotype were run. Results indicated that ketamine treated SERT $\%^{-}$subjects reared more overall compared to saline treated SERT $\%^{-}$counterparts $(\operatorname{adj} p=.015)$, whereas there were no differences between ketamine and saline treated WT subjects (see figure 17).

Figure 14. Acute Impact of Diazepam on Rearing Behaviour

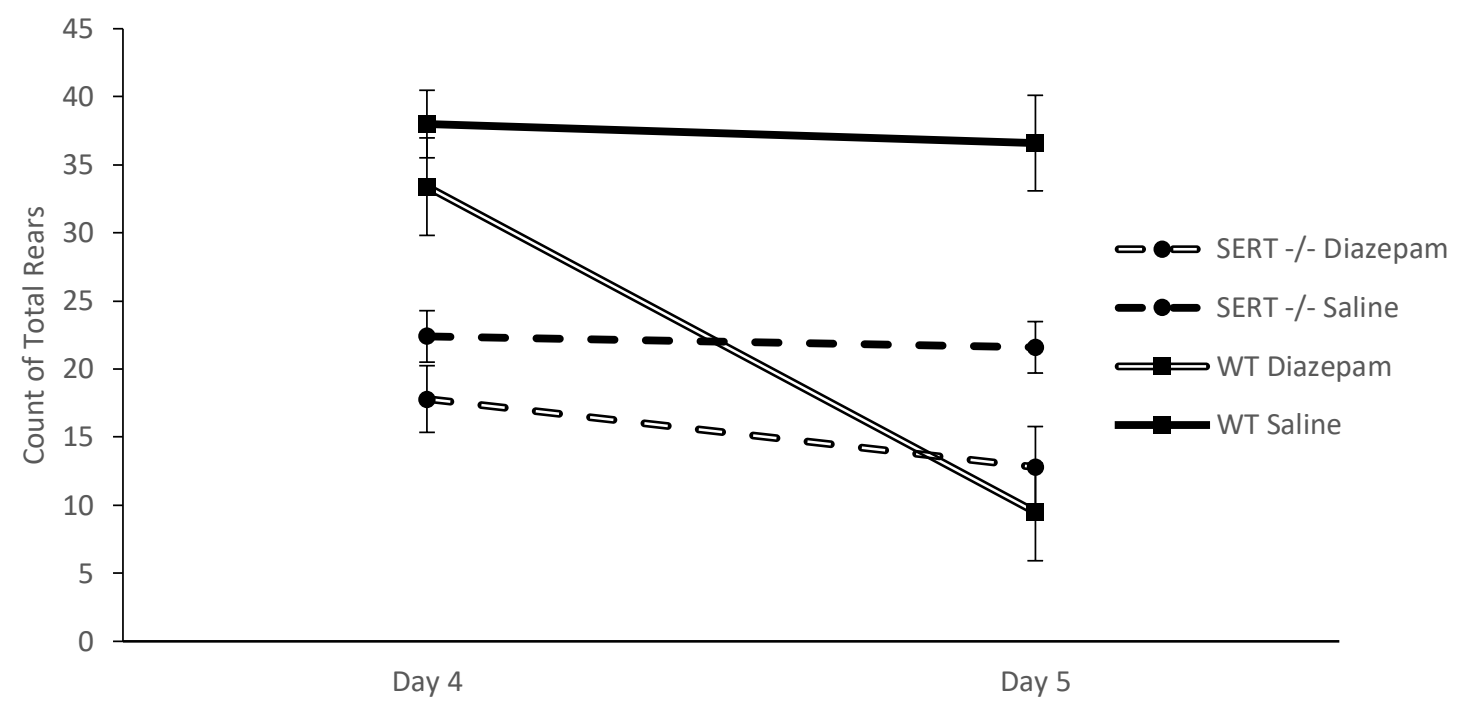

Figure 14. Means and \pm SEM of the acute impact of diazepam on rearing behaviour. Consecutive days are presented on the $x$ axis, and total number of rears made is depicted through the $y$ axis. 
Figure 15. Long-Term Impact of Diazepam on Rearing Behaviour

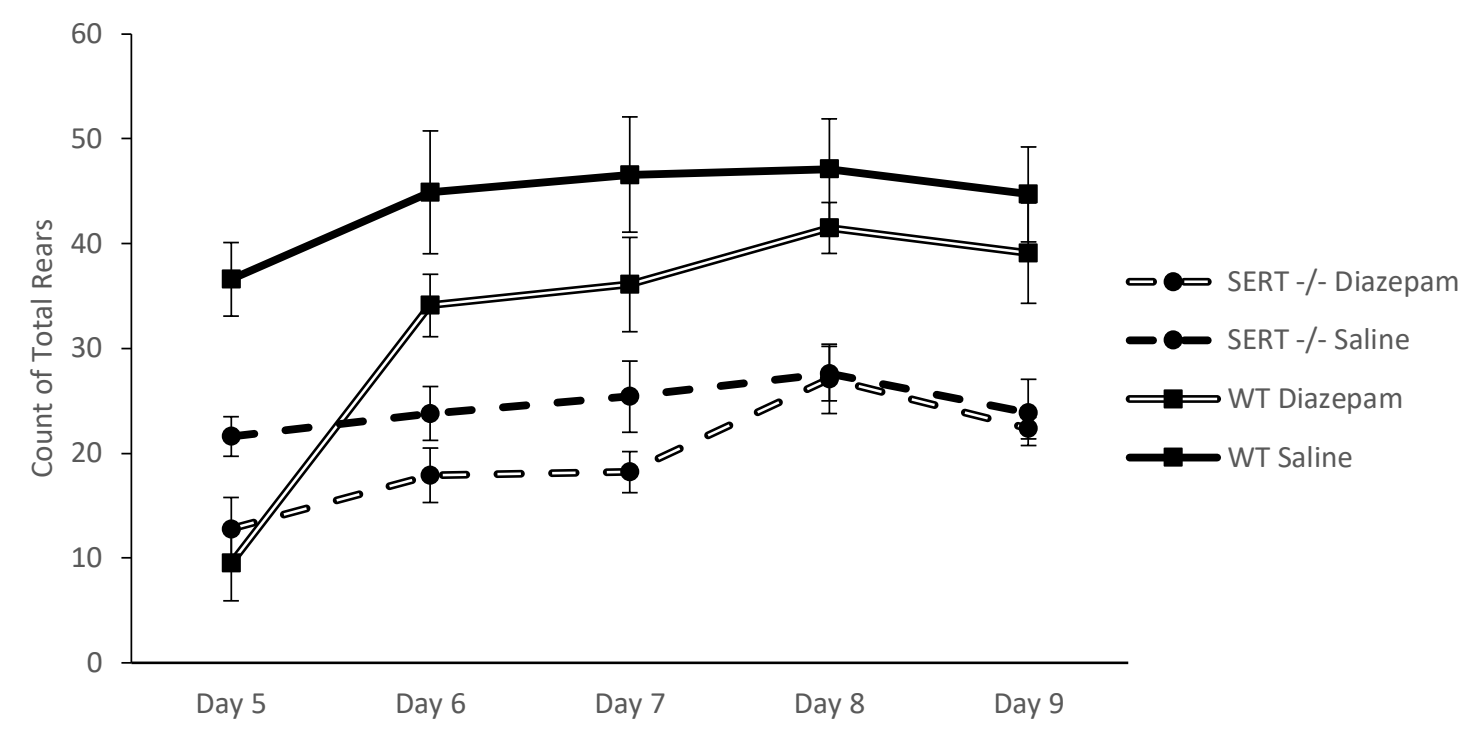

Figure 15. Means and \pm SEM of the long-term impact of diazepam on rearing behaviour. Consecutive days are presented on the $x$ axis, and total number of rears made is depicted through the $y$ axis.

Figure 16. Acute Impact of Ketamine on Rearing Behaviour

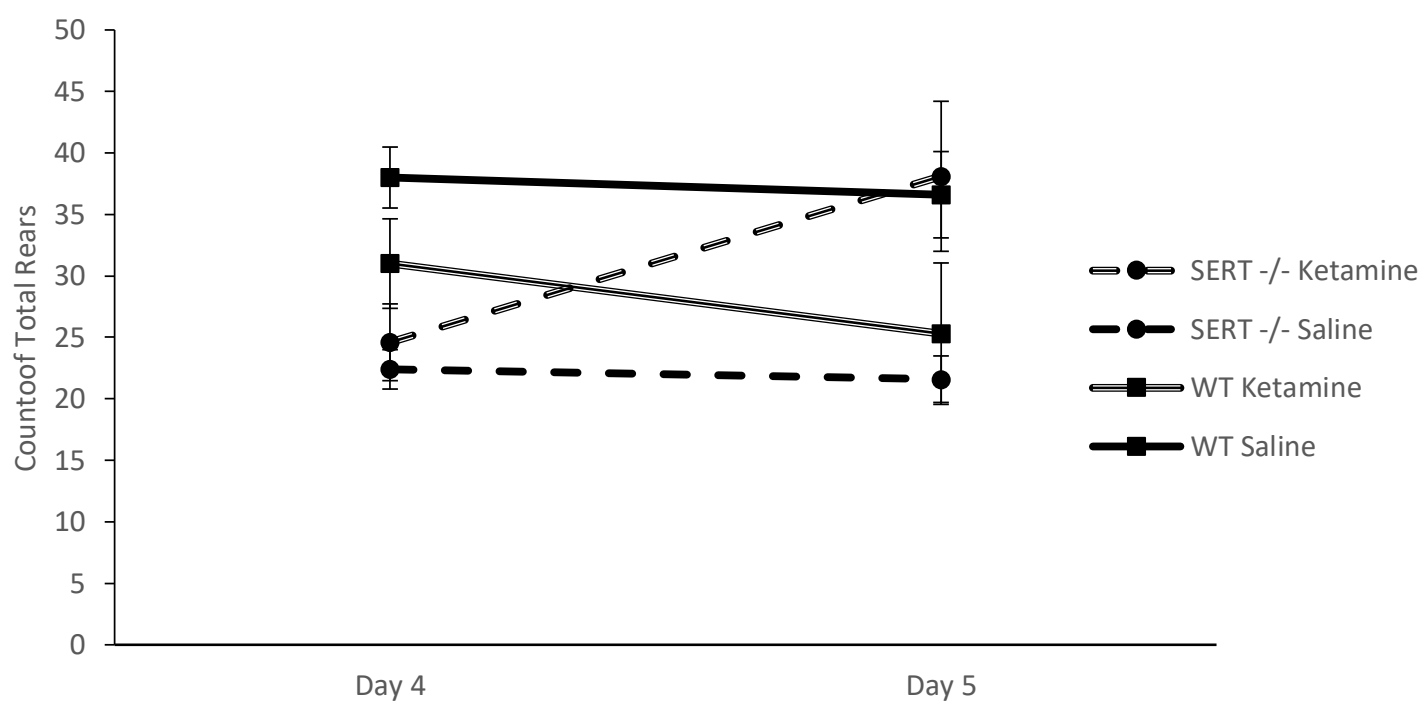

Figure 16. Means and \pm SEM of the acute impact of ketamine on rearing behaviour. Consecutive days are presented on the $x$ axis, and total number of rears made is depicted through the $y$ axis. 
Figure 17. Long-Term Impact of Ketamine on Rearing Behaviour

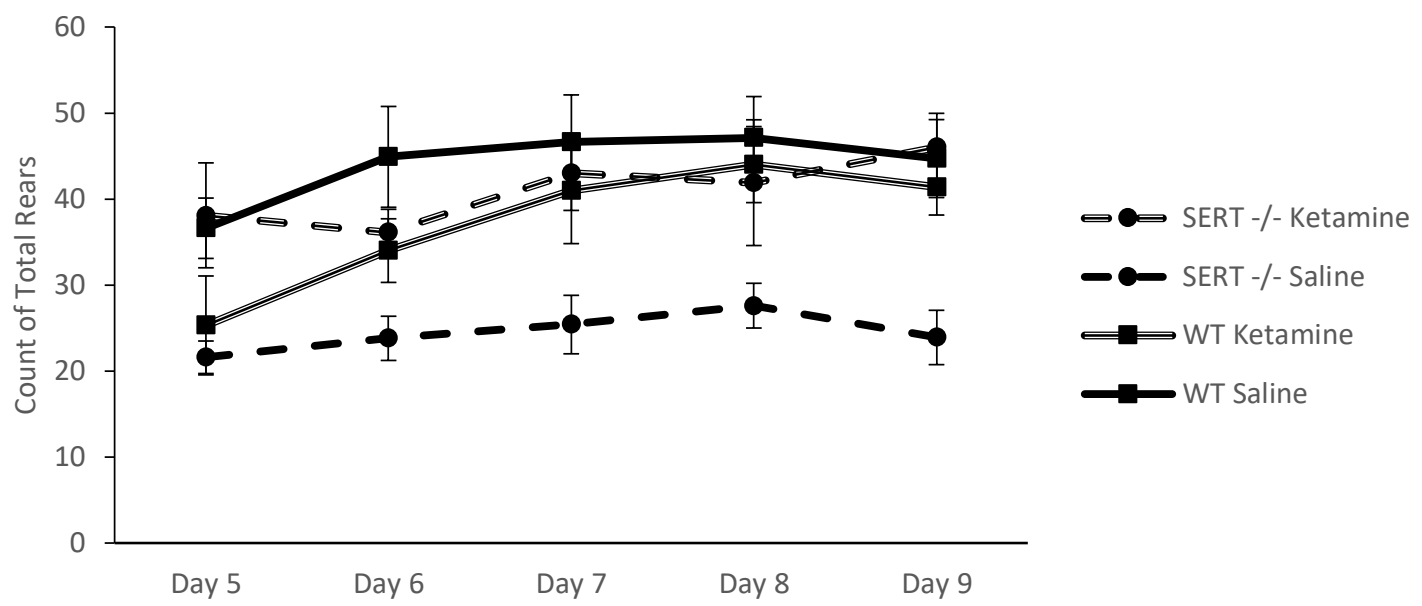

Figure 17. Means and \pm SEM of the long-term impact of ketamine on rearing behaviour. Consecutive days are presented on the $x$ axis, and total number of rears made is depicted through the $y$ axis. 
Table 3. Summary of Analyses on Rearing Behaviour

\begin{tabular}{|c|c|c|c|}
\hline Analysis & Model Predictors & F Statistic & p-value \\
\hline \multirow[t]{3}{*}{ Genotype Differences } & Day & $F(3,135)=28.48$ & $<.001 * * *$ \\
\hline & Genotype & $F(1,45)=14.80$ & $<.001 * * *$ \\
\hline & Day*Genotype & $F(3,135)=5.19$ & $.002 * *$ \\
\hline \multirow[t]{7}{*}{ Diazepam Acute } & Day & $F(1,28)=27.00$ & $<.001 * * *$ \\
\hline & Genotype & $F(1,28)=16.33$ & $<.001 * * *$ \\
\hline & Treatment & $F(1,28)=18.08$ & $<.001 * * *$ \\
\hline & Day*Genotype & $F(1,28)=10.71$ & $.003 * *$ \\
\hline & Day*Treatment & $F(1,28)=19.96$ & $<.001 * * *$ \\
\hline & Genotype*Treatment & $F(1,28)=2.96$ & .097 \\
\hline & Day*Genotype*Treatment & $F(1,28)=9.27$ & $.005 * *$ \\
\hline \multirow[t]{7}{*}{ Diazepam Long-Term } & Day & $F(4,112)=26.67$ & $<.001 * * *$ \\
\hline & Genotype & $F(1,28)=32.29$ & $<.001 * * *$ \\
\hline & Treatment & $F(1,28)=8.80$ & $.006^{* *}$ \\
\hline & Day*Genotype & $F(4,112)=6.23$ & $<.001 * * *$ \\
\hline & Day*Treatment & $F(4,112)=6.81$ & $<.001 * * *$ \\
\hline & Genotype*Treatment & $F(1,28)=1.61$ & .214 \\
\hline & Day*Genotype*Treatment & $F(4,112)=1.84$ & .125 \\
\hline \multirow[t]{7}{*}{ Ketamine Acute } & Day & $F(1,26)=0.46$ & .503 \\
\hline & Genotype & $F(1,26)=2.99$ & .096 \\
\hline & Treatment & $F(1,26)=0.00$ & .978 \\
\hline & Day*Genotype & $F(1,26)=5.81$ & $.023 *$ \\
\hline & Day*Treatment & $F(1,26)=1.45$ & .239 \\
\hline & Genotype*Treatment & $F(1,26)=7.06$ & $.013 *$ \\
\hline & Day*Genotype*Treatment & $F(1,26)=5.04$ & $.033 *$ \\
\hline \multirow[t]{7}{*}{ Ketamine Long-Term } & Day & $F(4,104)=6.77$ & $<.001 * * *$ \\
\hline & Genotype & $F(1,26)=4.86$ & $.037 *$ \\
\hline & Treatment & $F(1,26)=1.90$ & .180 \\
\hline & Day*Genotype & $F(4,104)=1.57$ & .187 \\
\hline & Day*Treatment & $F(4,104)=1.14$ & .334 \\
\hline & Genotype*Treatment & $F(1,26)=10.76$ & $.003 * *$ \\
\hline & Day*Genotype*Treatment & $F(4,104)=0.39$ & .819 \\
\hline
\end{tabular}

Note. ${ }^{*} p<.05, * * p<.01, * * * p<.001$ 
A total of 2 trials were removed from analysis of alley behaviour, 1 due to technical error, and the other due to the subject falling off the alley. As retrieval score and latency were manually recorded, and gathered prior to the subject falling, these measures were not impacted.

\section{Duration in Alley 4}

When investigating genotype differences in duration spent in alley 4, Mauchly's test of sphericity was violated, therefore a Greenhouse-Geisser correction was applied. Results showed a significant main effect of day and genotype. The duration spent in alley 4 increased across the days, and WT subjects spent longer in this alley compared to SERT $\%$ in general (see figure 18). No interactions were found (see table 4).

Figure 18. Genotype Differences in Cumulative Duration in Alley 4

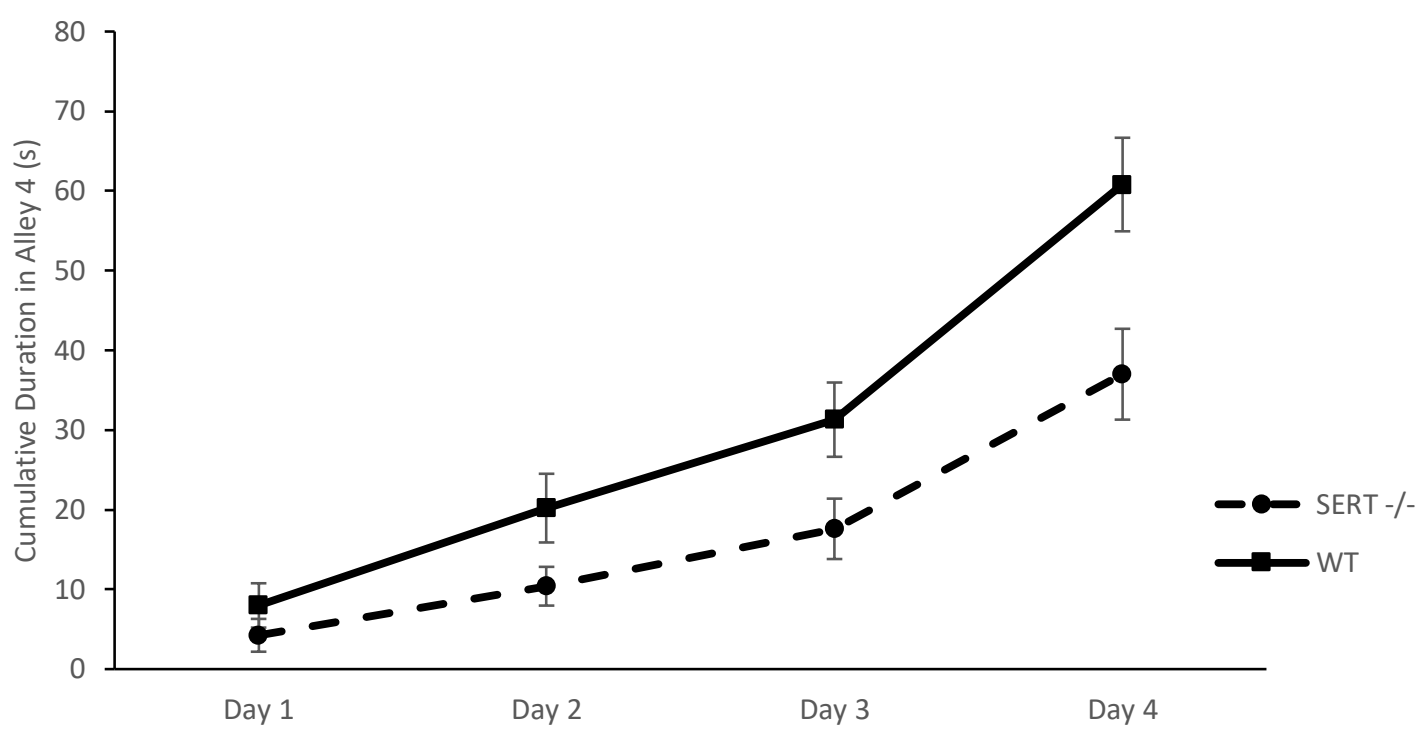

Figure 18. Means and \pm SEM of genotype differences in duration spent in alley 4 on the first four days of testing (Total trial duration 300s). Consecutive days are presented on the $x$ axis, and cumulative duration in second is depicted through the $y$ axis.

Diazepam had no acute impact on duration spent in alley 4 . However, a main effect of genotype was revealed (see table 4), whereby WT subjects spent longer in alley 4 compared to SERT $\%$, further supporting the genotype differences found above (see appendix $C$ ).

Investigation of the long-term impact of diazepam also showed a main effect of genotype and revealed a day*treatment and day*genotype*treatment interaction (see figure 
19). Further investigation of each treatment on each genotype at each day was conducted. Results indicated that at each day, diazepam treated subjects did not differ from their saline counterparts for either genotype.

Figure 19. Long-Term Impact of Diazepam on Cumulative Duration in Alley 4

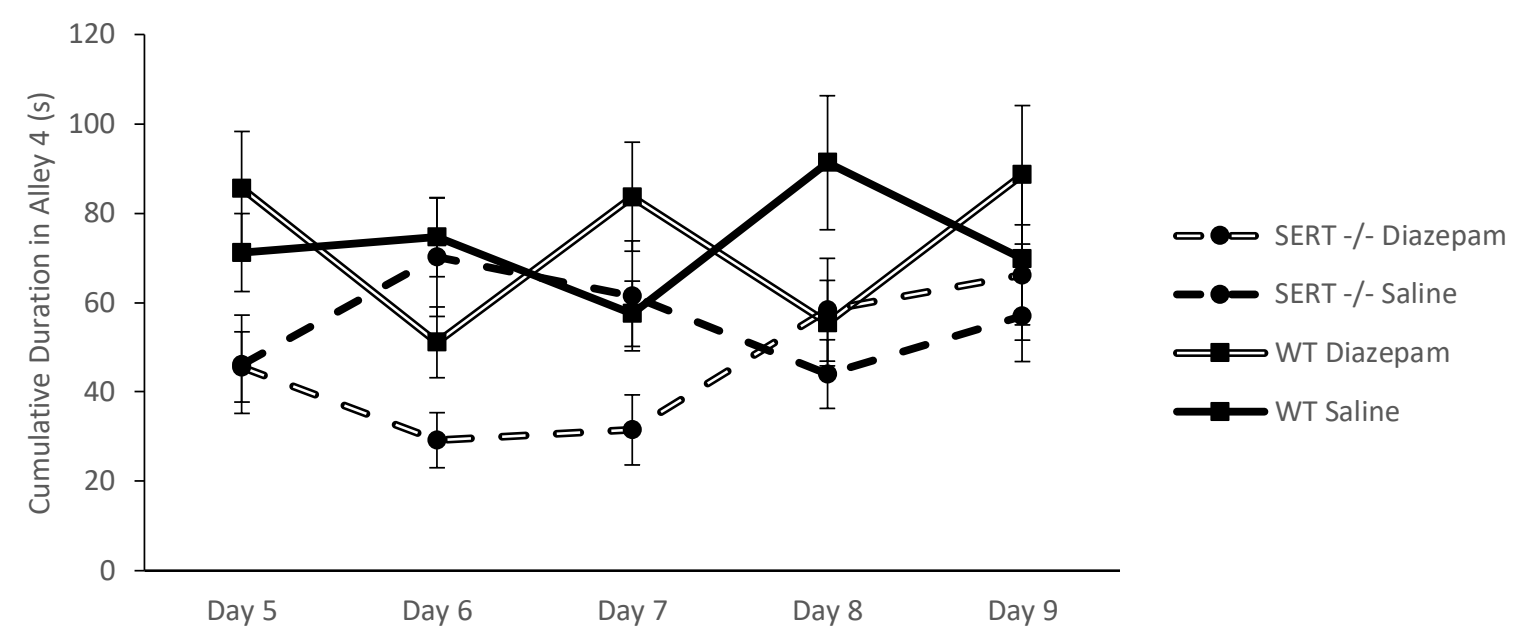

Figure 19. Means and \pm SEM of the long-term impact of diazepam on duration spent in alley 4 (Total trial duration 300s). Consecutive days are presented on the $x$ axis, and cumulative duration in seconds is depicted through the $y$ axis.

There was no acute or long-term impact of ketamine on the duration spent in alley 4. A main effect of genotype supported previous findings that WT subjects spent more time in alley 4 than SERT $\%$ subjects (see table 4 \& appendix $C$ ). 
Table 4. Summary of Analyses on Cumulative Duration in Alley 4

\begin{tabular}{|c|c|c|c|}
\hline Analysis & Model Predictors & F Statistic & p-value \\
\hline \multirow[t]{3}{*}{ Genotype Differences } & Day & $F(2.28,102.50)=54.84$ & $<.001 * * *$ \\
\hline & Genotype & $F(1,45)=10.10$ & $.003 * *$ \\
\hline & Day*Genotype & $F(2.28,102.50)=2.89$ & .53 \\
\hline \multirow[t]{7}{*}{ Diazepam Acute } & Day & $F(1,28)=3.53$ & .071 \\
\hline & Genotype & $F(1,28)=10.44$ & $.003 * *$ \\
\hline & Treatment & $F(1,28)=0.71$ & 405 \\
\hline & Day*Genotype & $F(1,28)=0.57$ & .455 \\
\hline & Day*Treatment & $F(1,28)=0.01$ & .918 \\
\hline & Genotype*Treatment & $F(1,28)=0.04$ & .852 \\
\hline & Day*Genotype*Treatment & $F(1,28)=1.20$ & .283 \\
\hline \multirow[t]{7}{*}{ Diazepam Long-Term } & Day & $F(4,104)=1.41$ & .236 \\
\hline & Genotype & $F(1,26)=8.54$ & $.007 * *$ \\
\hline & Treatment & $F(1,26)=0.40$ & .532 \\
\hline & Day*Genotype & $F(4,104)=0.31$ & .871 \\
\hline & Day*Treatment & $F(4,104)=2.74$ & $.032^{*}$ \\
\hline & Genotype*Treatment & $F(1,26)=0.40$ & .534 \\
\hline & Day*Genotype*Treatment & $F(4,104)=3.65$ & $.008 * *$ \\
\hline \multirow[t]{7}{*}{ Ketamine Acute } & Day & $F(1,26)=3.12$ & .089 \\
\hline & Genotype & $F(1,26)=6.51$ & $.017^{*}$ \\
\hline & Treatment & $F(1,26)=0.59$ & .450 \\
\hline & Day*Genotype & $F(1,26)=0.48$ & .496 \\
\hline & Day*Treatment & $F(1,26)=0.00$ & .963 \\
\hline & Genotype*Treatment & $F(1,26)=0.20$ & .655 \\
\hline & Day*Genotype*Treatment & $F(1,26)=0.15$ & .705 \\
\hline \multirow[t]{7}{*}{ Ketamine Long-Term } & Day & $F(4,96)=1.19$ & .319 \\
\hline & Genotype & $F(1,24)=2.13$ & .157 \\
\hline & Treatment & $F(1,24)=0.21$ & .651 \\
\hline & Day*Genotype & $F(4,96)=1.72$ & .151 \\
\hline & Day*Treatment & $F(4,96)=1.23$ & .304 \\
\hline & Genotype*Treatment & $F(1,24)=0.25$ & .624 \\
\hline & Day*Genotype*Treatment & $F(4,96)=0.14$ & .968 \\
\hline
\end{tabular}

Note. ${ }^{*} p<.05,{ }^{* *} p<.01, * * * p<.001$ 


\section{Latency to Alley 4}

No genotype differences were found when investigating latency to alley 4 on days 1-4. A main effect of day was found whereby latency to enter this alley decreased across the days (see table 5 \& appendix $D$ ).

Diazepam treatment had no acute impact on latency to alley 4 , however a main effect of day and day*genotype interaction was found, whereby latency to enter decreased across the days, more so for SERT \% (see table $5 \&$ appendix $D$ ).

When investigating the long-term impact, Mauchly's test of sphericity was violated and a Greenhouse-Geisser correction was applied. Analysis revealed a main effect of day, and day*treatment interaction (see figure 20). However, planned comparisons of treatment effects at each day indicated no significant difference between treatment groups across days 5-9.

Figure 20. Long-Term Impact of Diazepam on Latency to Enter Alley 4

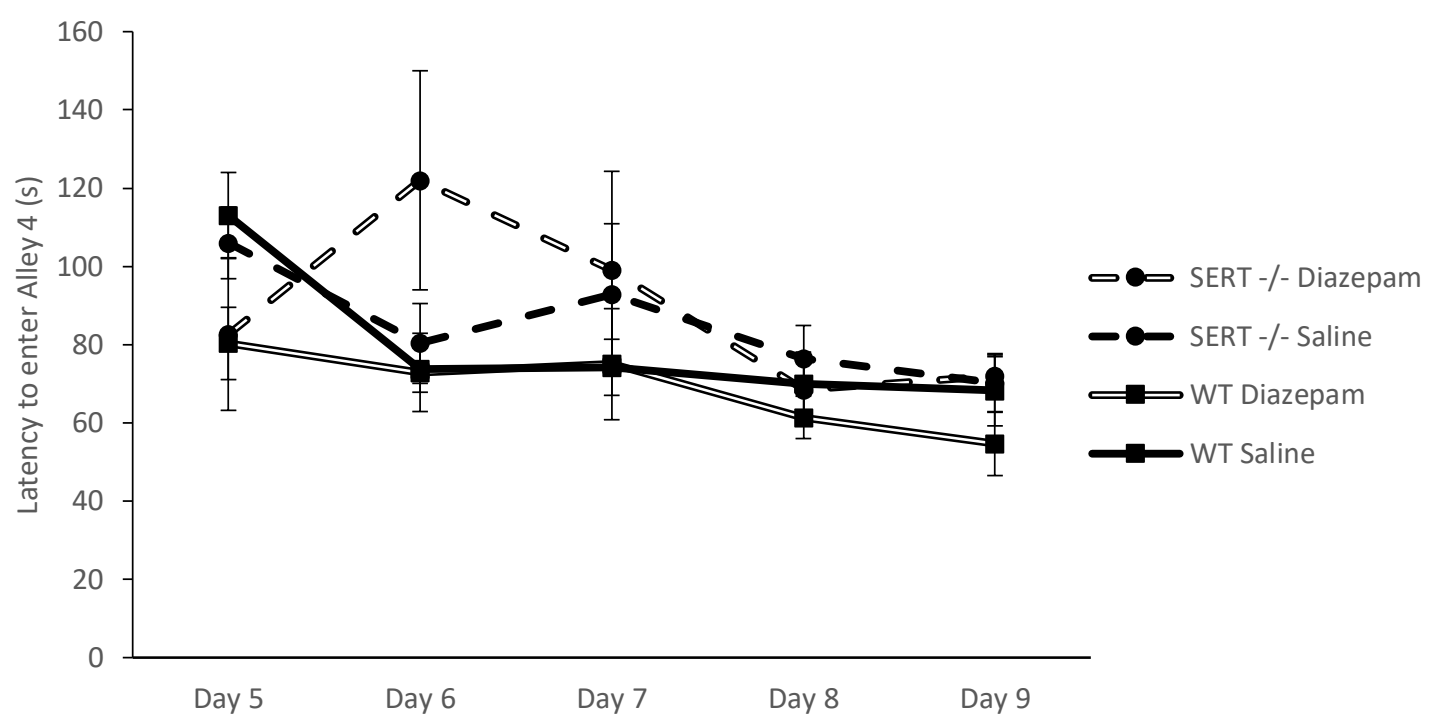

Figure 20. Means and \pm SEM of the long-term impact of diazepam on latency to alley 4 (Total trial duration 300s). Consecutive days are presented on the $x$ axis, and latency in seconds is depicted through the $y$ axis.

Ketamine treatment also had no immediate impact on latency to enter Alley 4 (see table 5). When investigating the long-term impact of treatment, a significant main effect of day and day*treatment interaction was found (see figure 21). However, follow up analysis found no significant differences between treatment groups at each day. 
Figure 21. Long-Term Impact of Ketamine on Latency to Enter Alley 4

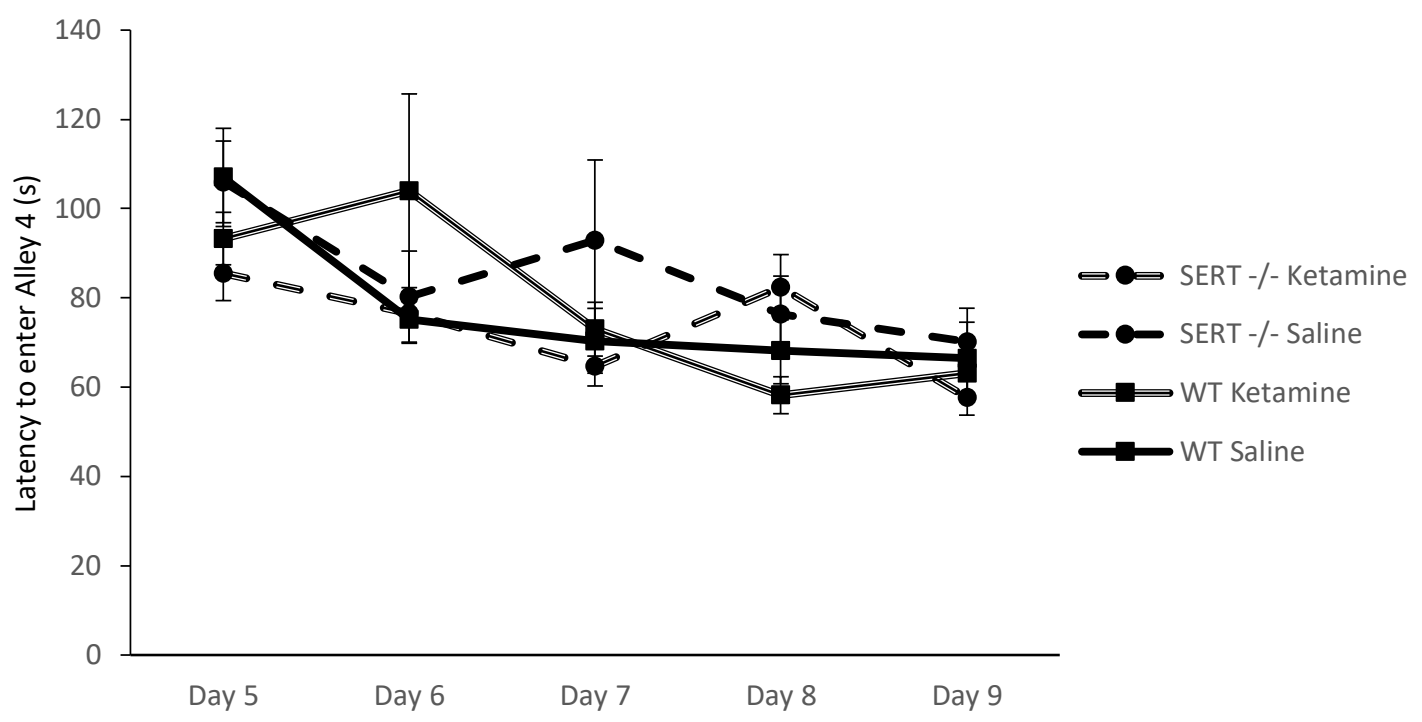

Figure 21. Means and \pm SEM of the long-term impact of ketamine on latency to alley 4 (Total trial duration 300s). Consecutive days are presented on the $x$ axis, and latency in seconds is depicted through the $y$ axis. 
Table 5. Summary of Analyses on Latency to Enter Alley 4

\begin{tabular}{|c|c|c|c|}
\hline Analysis & Model Predictors & F Statistic & p-value \\
\hline \multirow[t]{3}{*}{ Genotype Differences } & Day & $F(1,135)=42.26$ & $<.001 * * *$ \\
\hline & Genotype & $F(1,45)=1.12$ & .295 \\
\hline & Day*Genotype & $F(1,135)=0.381$ & .767 \\
\hline \multirow[t]{7}{*}{ Diazepam Acute } & Day & $F(1,28)=5.02$ & $.033^{*}$ \\
\hline & Genotype & $F(1,28)=1.04$ & .318 \\
\hline & Treatment & $F(1,28)=1.29$ & .267 \\
\hline & Day*Genotype & $F(1,28)=5.02$ & $.033^{*}$ \\
\hline & Day*Treatment & $F(1,28)=0.94$ & .339 \\
\hline & Genotype*Treatment & $F(1,28)=0.20$ & .658 \\
\hline & Day*Genotype*Treatment & $F(1,28)=0.15$ & .704 \\
\hline \multirow[t]{7}{*}{ Diazepam Long-Term } & Day & $F(2.47,64.18)=5.82$ & $.003 * *$ \\
\hline & Genotype & $F(1,26)=0.77$ & .387 \\
\hline & Treatment & $F(1,26)=0.01$ & .940 \\
\hline & Day*Genotype & $F(2.47,64.18)=1.30$ & .283 \\
\hline & Day*Treatment & $F(2.47,64.18)=3.39$ & $.031 *$ \\
\hline & Genotype*Treatment & $F(1,26)=0.79$ & .384 \\
\hline & Day*Genotype*Treatment & $F(2.47,64.18)=0.82$ & .466 \\
\hline \multirow[t]{7}{*}{ Ketamine Acute } & Day & $F(1,26)=4.04$ & .055 \\
\hline & Genotype & $F(1,26)=0.07$ & .790 \\
\hline & Treatment & $F(1,26)=0.45$ & .510 \\
\hline & Day*Genotype & $F(1,26)=0.91$ & .348 \\
\hline & Day*Treatment & $F(1,26)=0.94$ & .341 \\
\hline & Genotype*Treatment & $F(1,26)=0.10$ & .760 \\
\hline & Day*Genotype*Treatment & $F(1,26)=0.32$ & .576 \\
\hline \multirow[t]{7}{*}{ Ketamine Long-Term } & Day & $F(4,96)=12.86$ & $<.001 * * *$ \\
\hline & Genotype & $F(1,24)=0.04$ & .846 \\
\hline & Treatment & $F(1,24)=0.42$ & .521 \\
\hline & Day*Genotype & $F(4,96)=2.41$ & .055 \\
\hline & Day*Treatment & $F(4,96)=3.19$ & $.016^{*}$ \\
\hline & Genotype*Treatment & $F(1,24)=0.3$ & .854 \\
\hline & Day*Genotype*Treatment & $F(4,96)=1.45$ & .224 \\
\hline
\end{tabular}

Note. ${ }^{*} p<.05, * * p<.01, * * * p<.001$ 


\section{Duration in Alley 3}

To account for possible floor effects when investigating alley 4 behaviour, investigation of behaviour in alley 3 was also conducted.

Analysis of genotype differences in duration spent in alley 3 showed a main effect of day and genotype, and a day*genotype interaction. As depicted in Figure 22, duration spent in alley 3 increased more for SERT $\%$ subjects across the days, however WT spent longer in this alley overall.

Figure 22. Genotype Differences in Cumulative Duration in Alley 3

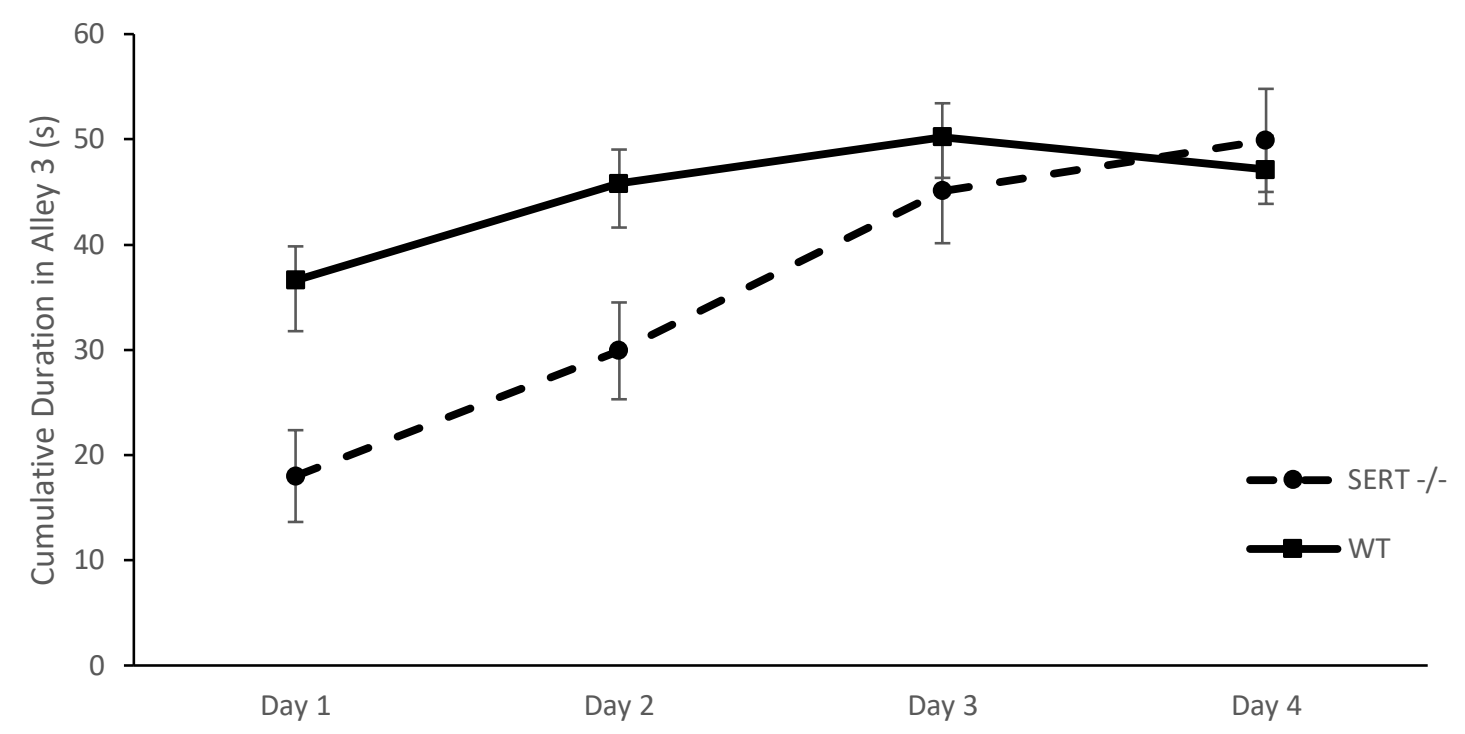

Figure 22. Means and \pm SEM of genotype differences in duration spent in alley 3 on the first four days of testing (Total trial duration 300s). Consecutive days are presented on the $x$ axis, and cumulative duration in second is depicted through the $y$ axis.

Investigation into the acute impact of diazepam revealed a significant day*treatment interaction (see figure 23). Planned comparisons of treatments at each day indicated no differences between treatments on day 4 , whereas on day 5 diazepam treated subjects spent an increased duration spent in alley 3 compared to controls (adj $p=.009$ ).

Further investigation into the long-term impact of diazepam similarly revealed a significant day*treatment interaction. Follow up analysis showed a significant difference between diazepam and saline treated subjects on day $5(\operatorname{adj} p=.020)$, and no difference between treatment groups on days 6-9 (See figure 24).

Similar to alley 4, ketamine had no acute or long-term impact on the duration spent in alley 3 (see table $6 \&$ appendix E). 
Figure 23. Acute Impact of Diazepam on Cumulative Duration in Alley 3

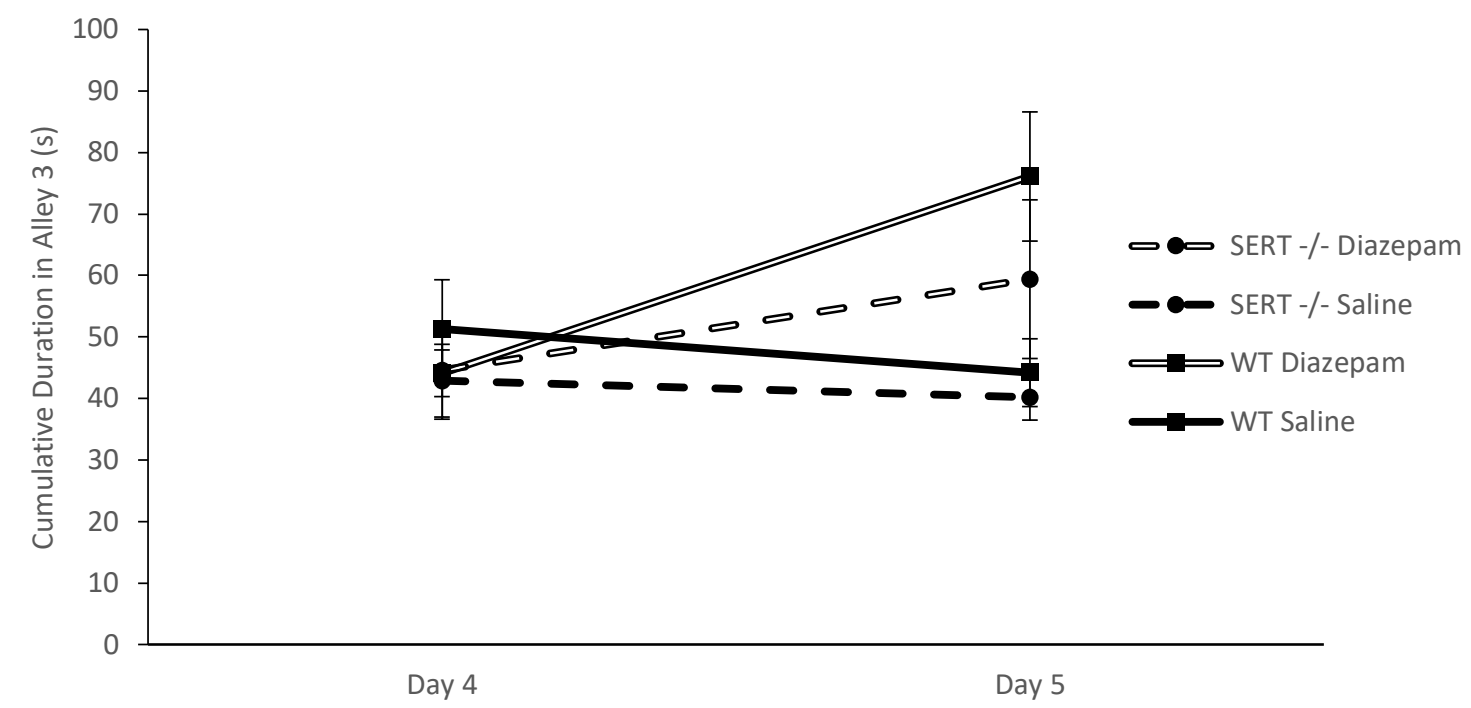

Figure 23. Means and \pm SEM of the acute impact of diazepam on duration spent in alley 3 (Total trial duration 300s). Consecutive days are presented on the $x$ axis, and cumulative duration in seconds is depicted through the $y$ axis.

Figure 24. Long-Term Impact of Diazepam on Cumulative Duration in Alley 3

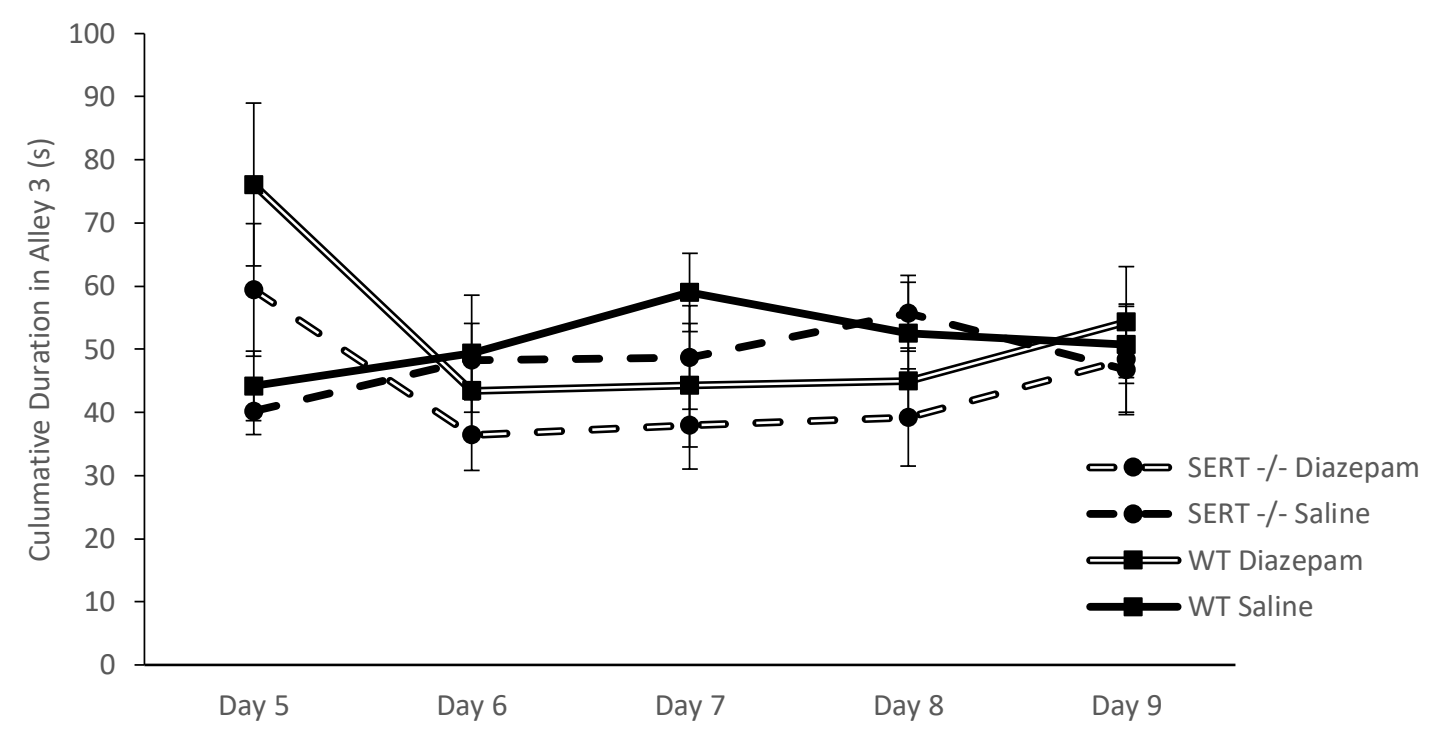

Figure 24. Means and \pm SEM of the long-term impact of diazepam on duration spent in alley 3 (Total trial duration 300s). Consecutive days are presented on the $x$ axis, and cumulative duration in seconds is depicted through the $y$ axis. 
Table 6. Summary of Analyses on Cumulative Duration in Alley 3

\begin{tabular}{|c|c|c|c|}
\hline Analysis & Model Predictors & F Statistic & p-value \\
\hline \multirow[t]{3}{*}{ Genotype Differences } & Day & $F(3,135)=11.29$ & $<.001 * * *$ \\
\hline & Genotype & $F(1,45)=6.64$ & $.013 *$ \\
\hline & Day*Genotype & $F(3,135)=2.82$ & .041 \\
\hline \multirow[t]{7}{*}{ Diazepam Acute } & Day & $F(1,28)=3.39$ & .076 \\
\hline & Genotype & $F(1,28)=0.93$ & .343 \\
\hline & Treatment & $F(1,28)=3.67$ & .066 \\
\hline & Day*Genotype & $F(1,28)=0.49$ & .489 \\
\hline & Day*Treatment & $F(1,28)=6.53$ & $.016^{*}$ \\
\hline & Genotype*Treatment & $F(1,28)=0.09$ & .765 \\
\hline & Day*Genotype*Treatment & $F(1,28)=0.84$ & .366 \\
\hline \multirow[t]{7}{*}{ Diazepam Long-Term } & Day & $F(4,104)=1.21$ & .310 \\
\hline & Genotype & $F(1,26)=1.04$ & .317 \\
\hline & Treatment & $F(1,26)=0.11$ & .745 \\
\hline & Day*Genotype & $F(4,104)=0.13$ & .970 \\
\hline & Day*Treatment & $F(4,104)=3.81$ & $.006 * *$ \\
\hline & Genotype*Treatment & $F(1,26)=0.58$ & .455 \\
\hline & Day*Genotype*Treatment & $F(4,104)=0.14$ & .966 \\
\hline \multirow[t]{7}{*}{ Ketamine Acute } & Day & $F(1,26)=1.93$ & .177 \\
\hline & Genotype & $F(1,26)=0.24$ & .627 \\
\hline & Treatment & $F(1,26)=2.36$ & .137 \\
\hline & Day*Genotype & $F(1,26)=0.30$ & .588 \\
\hline & Day*Treatment & $F(1,26)=0.24$ & .632 \\
\hline & Genotype*Treatment & $F(1,26)=1.44$ & .241 \\
\hline & Day*Genotype*Treatment & $F(1,26)=0.68$ & .418 \\
\hline \multirow[t]{7}{*}{ Ketamine Long-Term } & Day & $F(4,96)=0.42$ & .794 \\
\hline & Genotype & $F(1,24)=0.00$ & .952 \\
\hline & Treatment & $F(1,24)=0.55$ & .466 \\
\hline & Day*Genotype & $F(4,96)=0.36$ & .834 \\
\hline & Day*Treatment & $F(4,96)=0.83$ & .510 \\
\hline & Genotype*Treatment & $F(1,24)=0.06$ & .807 \\
\hline & Day*Genotype*Treatment & $F(4,96)=0.10$ & .982 \\
\hline
\end{tabular}

Note. $* p<.05, * * p<.01, * * * p<.001$ 


\section{Latency to Alley 3}

When investigating genotype differences and the long-term impact of diazepam on latency to alley 3, Mauchly's test of sphericity was violated, thus a Greenhouse-Geisser correction was applied. No genotype differences or treatment effects of diazepam and ketamine were found, both immediately and long-term. However main effects of day were found when investigating genotype differences and the long-term impact of ketamine, indicating latency to enter this alley decreased across the days (see table 7 \& appendix $F$ ).

\section{Retrieval Score}

When investigating genotype differences in retrieval score violated Mauchly's test of Sphericity, therefore a Greenhouse-Geisser correction was applied. Analysis revealed a significant main effect of day and genotype. As seen in Figure 25, retrieval scores increased across the first 4 days of testing, and WT subjects had significantly higher scores overall.

Figure 25. Genotype Differences in Retrieval Score

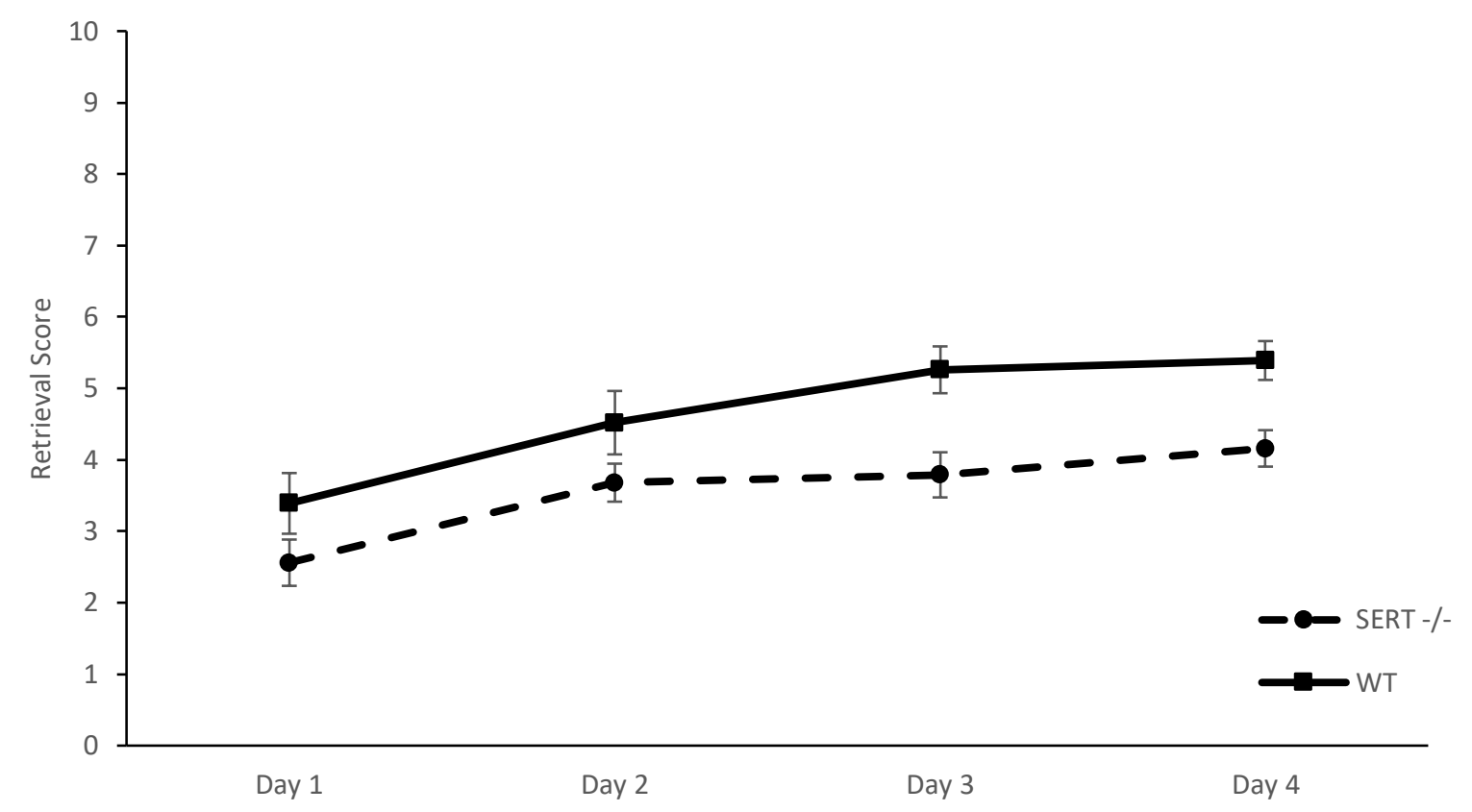

Figure 25. Means and \pm SEM of genotype differences in retrieval score on the first four days of testing. Consecutive days are presented on the $x$ axis, and score is depicted through the $y$ axis. 
Table 7. Summary of Analyses on Latency to Enter Alley 3

\begin{tabular}{|c|c|c|c|}
\hline Analysis & Model Predictors & F Statistic & p-value \\
\hline \multirow[t]{3}{*}{ Genotype Differences } & Day & $F(2.18,98.31)=14.36$ & $<.001 * * *$ \\
\hline & Genotype & $F(1,45)=2.48$ & .122 \\
\hline & Day*Genotype & $F(2.18,98.31)=1.06$ & .355 \\
\hline \multirow[t]{7}{*}{ Diazepam Acute } & Day & $F(1,28)=2.38$ & .134 \\
\hline & Genotype & $F(1,28)=0.00$ & .933 \\
\hline & Treatment & $F(1,28)=0.00$ & .943 \\
\hline & Day*Genotype & $F(1,28)=0.31$ & .581 \\
\hline & Day*Treatment & $F(1,28)=0.52$ & .478 \\
\hline & Genotype*Treatment & $F(1,28)=2.27$ & .143 \\
\hline & Day*Genotype*Treatment & $F(1,28)=0.29$ & .592 \\
\hline \multirow[t]{7}{*}{ Diazepam Long-Term } & Day & $F(2.20,57.07)=3.03$ & .052 \\
\hline & Genotype & $F(1,26)=0.43$ & .518 \\
\hline & Treatment & $F(1,26)=0.00$ & .940 \\
\hline & Day*Genotype & $F(2.20,57.07)=1.52$ & .225 \\
\hline & Day*Treatment & $F(2.20,57.07)=1.10$ & .346 \\
\hline & Genotype*Treatment & $F(1,26)=1.25$ & .275 \\
\hline & Day*Genotype*Treatment & $F(2.20,57.07)=0.74$ & .491 \\
\hline \multirow[t]{7}{*}{ Ketamine Acute } & Day & $F(1,26)=0.09$ & .770 \\
\hline & Genotype & $F(1,26)=1.06$ & .313 \\
\hline & Treatment & $F(1,26)=2.24$ & .147 \\
\hline & Day*Genotype & $F(1,26)=0.13$ & .725 \\
\hline & Day*Treatment & $F(1,26)=0.64$ & .430 \\
\hline & Genotype*Treatment & $F(1,26)=1.01$ & .324 \\
\hline & Day*Genotype*Treatment & $F(1,26)=0.11$ & .741 \\
\hline \multirow[t]{7}{*}{ Ketamine Long-Term } & Day & $F(4,96)=4.81$ & $.001 * * *$ \\
\hline & Genotype & $F(1,24)=0.61$ & .441 \\
\hline & Treatment & $F(1,24)=0.03$ & .862 \\
\hline & Day*Genotype & $F(4,96)=2.33$ & .061 \\
\hline & Day*Treatment & $F(4,96)=1.47$ & .281 \\
\hline & Genotype*Treatment & $F(1,24)=0.00$ & .971 \\
\hline & Day*Genotype*Treatment & $F(4,96)=1.39$ & .242 \\
\hline
\end{tabular}

Note. $* p<.05, * * p<.01, * * * p<.001$ 
Analysis of the immediate impact of diazepam on retrieval score showed main effects of day, genotype, and treatment, and a significant day*treatment interaction (see figure 26). Follow up comparisons indicated no difference between treatment groups on day 4, whereas diazepam significantly increased retrieval scores on day 5 compared to saline treated subjects $(\operatorname{adj} p<.001)$.

When investigating the long-term impact of diazepam Mauchly's test of Sphericity was violated, therefore a Greenhouse-Geisser correction applied. Results showed a significant main effect of day, genotype and treatment, as well as a significant day*treatment interaction (see figure 27). Follow up analysis conducted planned comparisons of each treatment group on each day. Retrieval scores were significantly higher for diazepam treated subjects compared to those treated with saline on day $5(\operatorname{adj} p<.001)$, whereas on days 6-9 there was no significant difference between treatments.

Ketamine had no immediate or long-term impact on retrieval score (see table $8 \&$ appendix $G)$.

Figure 26. Acute Impact of Diazepam on Retrieval Score

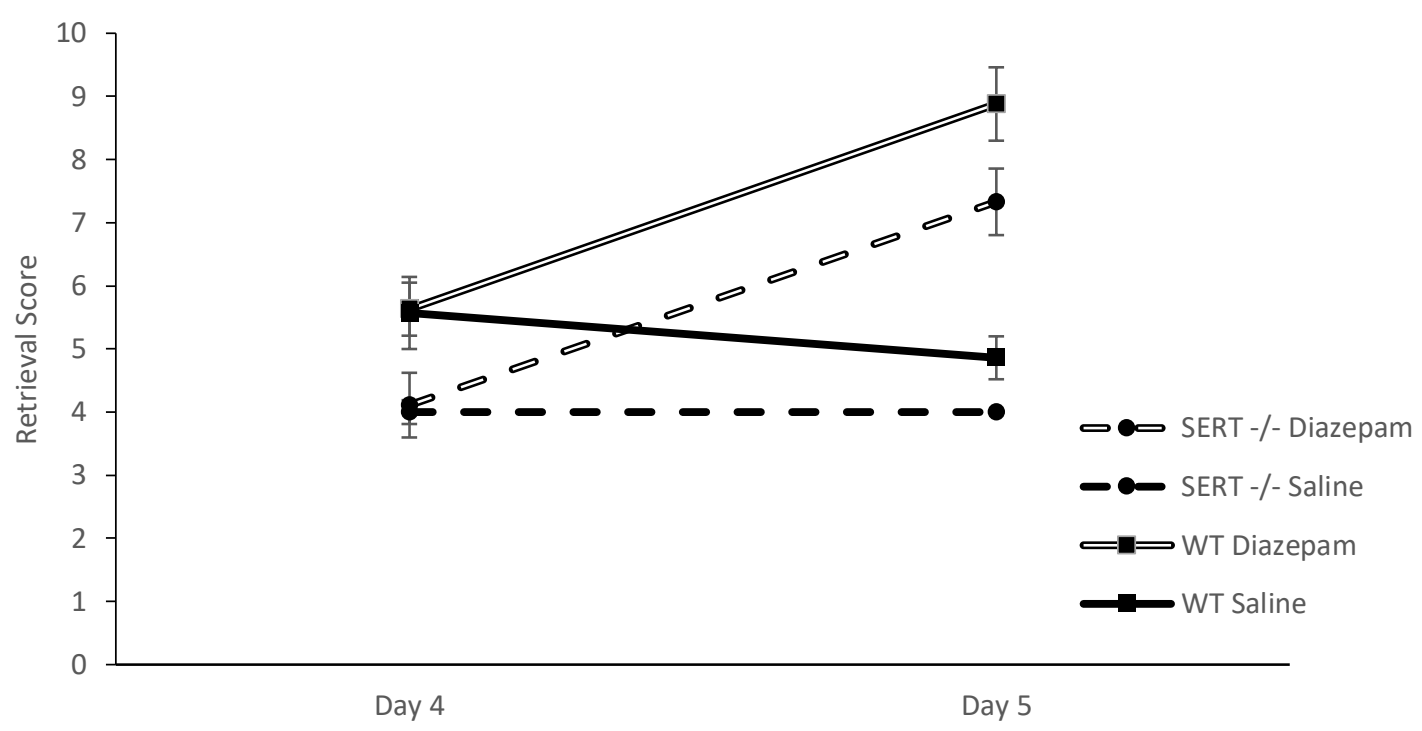

Figure 26. Means and \pm SEM of the acute impact of diazepam on retrieval score. Consecutive days are presented on the $x$ axis, and score is depicted through the $y$ axis. 
Figure 27. Long-Term Impact of Diazepam on Retrieval Score

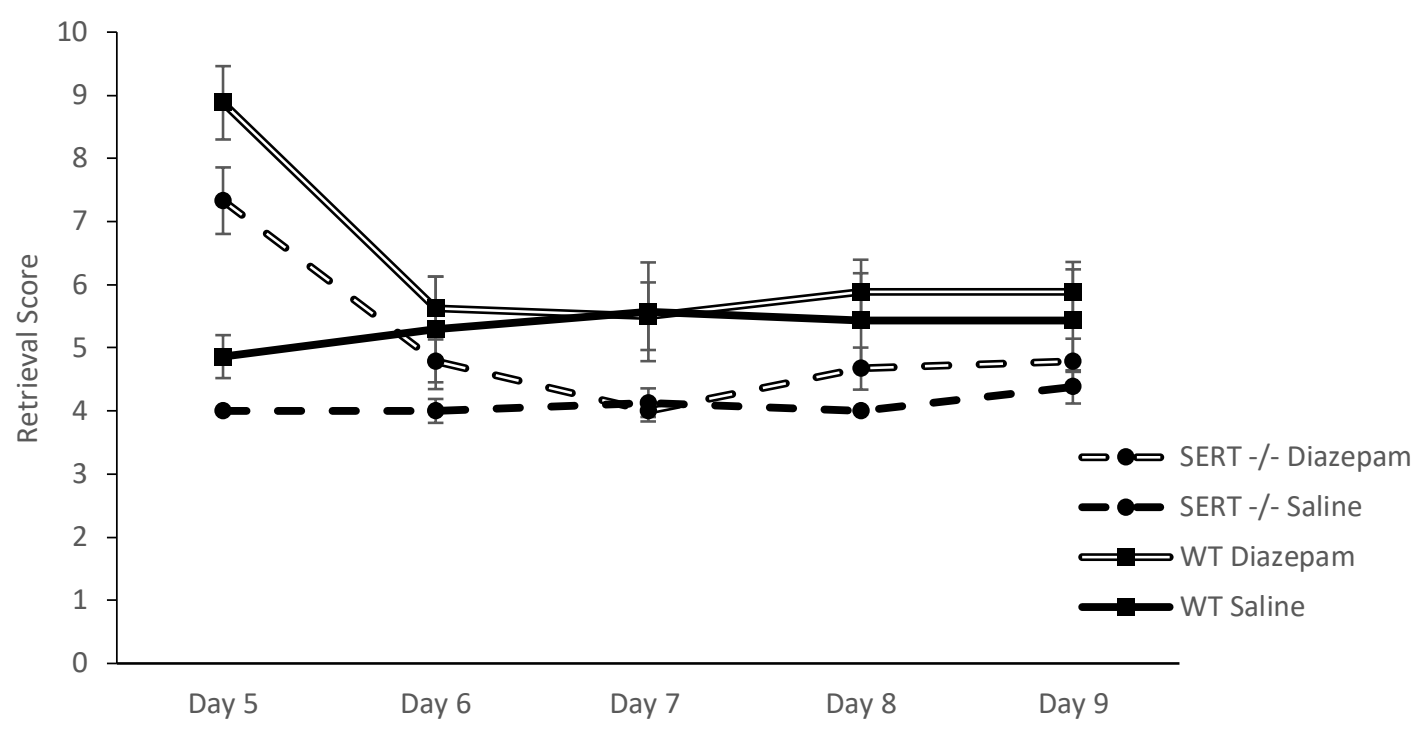

Figure 27. Means and \pm SEM of the long-term impact of diazepam on retrieval score. Consecutive days are presented on the $x$ axis, and score is depicted through the $y$ axis.

\section{Retrieval Latency}

When investigating genotype differences in retrieval latency, Mauchly's test of Sphericity was violated, and a Greenhouse-Geisser correction was applied. Results showed a main effect of day, whereby latency decreased across the days (see appendix $H$ ). No genotype differences or interaction was found (see table 9).

Acute diazepam treatment had no impact on retrieval latency, however, analysis revealed a main effect of day, and day*genotype interaction (see table 9). Investigation of the acute impact of ketamine treatment also revealed a main effect of day, as well as a day*genotype*treatment interaction, however no differences were found between groups on each day when a Bonferroni correction was applied (see appendix $H$ ).

Mauchly's test was violated when investigating long-term treatment effects of both drugs, therefore a Greenhouse-Geisser correction was used. Neither diazepam nor ketamine had no long-term impact on retrieval latency (see table 9). 
Table 8. Summary of Analyses on Retrieval Score

\begin{tabular}{|c|c|c|c|}
\hline Analysis & Model Predictors & F Statistic & p-value \\
\hline \multirow[t]{3}{*}{ Genotype Differences } & Day & $F(2.45,107.60)=19.17$ & $<.001 * * *$ \\
\hline & Genotype & $F(1,44)=9.15$ & $.004 * *$ \\
\hline & Day*Genotype & $F(2.45,107.60)=0.46$ & .672 \\
\hline \multirow[t]{7}{*}{ Diazepam Acute } & Day & $F(1,28)=30.45$ & $<.001 * * *$ \\
\hline & Genotype & $F(1,28)=14.41$ & $<.001 * * *$ \\
\hline & Treatment & $F(1,28)=27.07$ & $<.001 * * *$ \\
\hline & Day*Genotype & $F(1,28)=0.43$ & .516 \\
\hline & Day*Treatment & $F(1,28)=47.44$ & $<.001 * * *$ \\
\hline & Genotype*Treatment & $F(1,28)=0.19$ & .668 \\
\hline & Day*Genotype*Treatment & $F(1,28)=0.51$ & .483 \\
\hline \multirow[t]{7}{*}{ Diazepam Long-Term } & Day & $F(2.75,76.89)=14.08$ & $<.001 * * *$ \\
\hline & Genotype & $F(1,28)=10.46$ & $.003 * *$ \\
\hline & Treatment & $F(1,28)=7.28$ & $.012 *$ \\
\hline & Day*Genotype & $F(2.75,76.89)=0.29$ & .813 \\
\hline & Day*Treatment & $F(2.75,76.89)=22.46$ & $<.001 * * *$ \\
\hline & Genotype*Treatment & $F(1,28)=0.00$ & .974 \\
\hline & Day*Genotype*Treatment & $F(2.75,76.89)=0.44$ & .708 \\
\hline \multirow[t]{7}{*}{ Ketamine Acute } & Day & $F(1,26)=0.17$ & .685 \\
\hline & Genotype & $F(1,26)=2.92$ & .099 \\
\hline & Treatment & $F(1,26)=0.33$ & .574 \\
\hline & Day*Genotype & $F(1,26)=0.92$ & .347 \\
\hline & Day*Treatment & $F(1,26)=0.92$ & .347 \\
\hline & Genotype*Treatment & $F(1,26)=1.12$ & .300 \\
\hline & Day*Genotype*Treatment & $F(1,26)=0.17$ & .068 \\
\hline \multirow[t]{7}{*}{ Ketamine Long-Term } & Day & $F(2.53,65.65)=0.81$ & .521 \\
\hline & Genotype & $F(1,26)=0.35$ & .559 \\
\hline & Treatment & $F(1,26)=0.23$ & .638 \\
\hline & Day*Genotype & $F(2.53,65.65)=0.41$ & .712 \\
\hline & Day*Treatment & $F(2.53,65.65)=0.58$ & .600 \\
\hline & Genotype*Treatment & $F(1,26)=3.52$ & .072 \\
\hline & Day*Genotype*Treatment & $F(2.53,65.65)=1.27$ & .292 \\
\hline
\end{tabular}

Note. ${ }^{*} p<.05, * * p<.01, * * * p<.001$ 
Table 9. Summary of Analyses on Retrieval Latency

\begin{tabular}{|c|c|c|c|}
\hline Analysis & Model Predictors & F Statistic & p-value \\
\hline \multirow[t]{3}{*}{ Genotype Differences } & Day & $F(2.30,101.22)=63.93$ & $<.001 * * *$ \\
\hline & Genotype & $F(1,44)=0.01$ & .932 \\
\hline & Day*Genotype & $F(2.30,101.22)=0.99$ & .398 \\
\hline \multirow[t]{7}{*}{ Diazepam Acute } & Day & $F(1,28)=9.19$ & $.005^{* *}$ \\
\hline & Genotype & $F(1,28)=1.05$ & .317 \\
\hline & Treatment & $F(1,28)=1.96$ & .172 \\
\hline & Day*Genotype & $F(1,28)=6.99$ & $.013 *$ \\
\hline & Day*Treatment & $F(1,28)=0.65$ & .426 \\
\hline & Genotype*Treatment & $F(1,28)=0.47$ & .298 \\
\hline & Day*Genotype*Treatment & $F(1,28)=0.49$ & .491 \\
\hline \multirow[t]{7}{*}{ Diazepam Long-Term } & Day & $F(2.81,78.76)=2.29$ & .089 \\
\hline & Genotype & $F(1,28)=2.43$ & .563 \\
\hline & Treatment & $F(1,28)=0.04$ & .835 \\
\hline & Day*Genotype & $F(2.81,78.76)=0.67$ & .614 \\
\hline & Day*Treatment & $F(2.81,78.76)=0.79$ & .496 \\
\hline & Genotype*Treatment & $F(1,28)=0.20$ & .753 \\
\hline & Day*Genotype*Treatment & $F(2.81,78.76)=1.15$ & .331 \\
\hline \multirow[t]{7}{*}{ Ketamine Acute } & Day & $F(1,26)=10.03$ & $.004 * *$ \\
\hline & Genotype & $F(1,26)=0.24$ & .626 \\
\hline & Treatment & $F(1,26)=0.21$ & .650 \\
\hline & Day*Genotype & $F(1,26)=0.26$ & .611 \\
\hline & Day*Treatment & $F(1,26)=0.03$ & .871 \\
\hline & Genotype*Treatment & $F(1,26)=2.35$ & .137 \\
\hline & Day*Genotype*Treatment & $F(1,26)=5.68$ & $.024 *$ \\
\hline \multirow[t]{7}{*}{ Ketamine Long-Term } & Day & $F(1.32,34.38)=1.67$ & .162 \\
\hline & Genotype & $F(1,26)=1.49$ & .233 \\
\hline & Treatment & $F(1,26)=0.00$ & .987 \\
\hline & Day*Genotype & $F(1.32,34.38)=1.44$ & .246 \\
\hline & Day*Treatment & $F(1.32,34.38)=0.84$ & .398 \\
\hline & Genotype*Treatment & $F(1,26)=0.32$ & .578 \\
\hline & Day*Genotype*Treatment & $F(1.32,34.38)=0.30$ & .653 \\
\hline
\end{tabular}

Note. ${ }^{*} p<.05, * * p<.01, * * * p<.001$ 


\section{Locomotor Activity}

Locomotor activity was measured as distance travelled in the anticipation box. Due to technical error, 12 trials were removed from analysis.

When investigating genotype differences on days $1-4$, the results showed a main effect of day, where distance increased across the days (see appendix I). No genotype differences or interaction were found (see table 10).

Investigation into the immediate impact of diazepam on locomotor activity revealed a main effect of day and treatment. Day*treatment, and day*genotype*treatment interactions were also found. Following the analysis, planned comparisons of each treatment on each genotype on both days were run. No differences were found on day 4 between treatment groups for either genotype. On day 5 , there was no significant difference between treatments for the SERT $\%$ strain, whereas WT diazepam treated subjects travelled significantly less distance than their saline treated counterparts $(\operatorname{adj} p<.001)($ see figure 28$)$.

When investigating the long-term impact of diazepam on locomotor activity, Mauchly's test of sphericity was violated, and a Greenhouse-Geisser correction was applied. Results showed a main effect of day and treatment, whereby diazepam treated subjects travelled less distance on average across all days (figure 29).

Figure 28. Acute Impact of Diazepam on Locomotor Activity

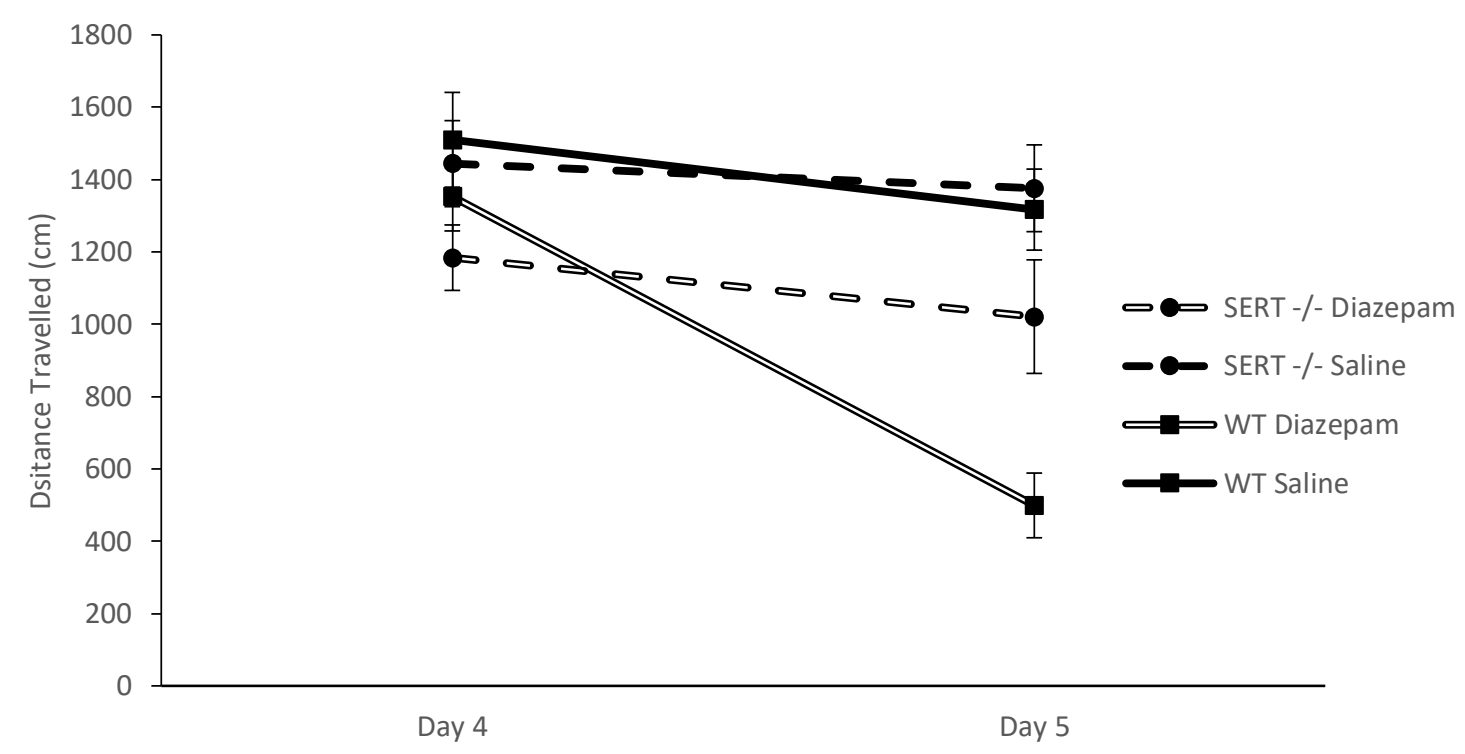

Figure 28. Means and \pm SEM of the acute impact of diazepam on locomotor activity. Consecutive days are presented on the $x$ axis, and distance travelled in $\mathrm{cm}$ is depicted through the $y$ axis. 
Figure 29. Long-Term Impact of Diazepam on Locomotor Activity

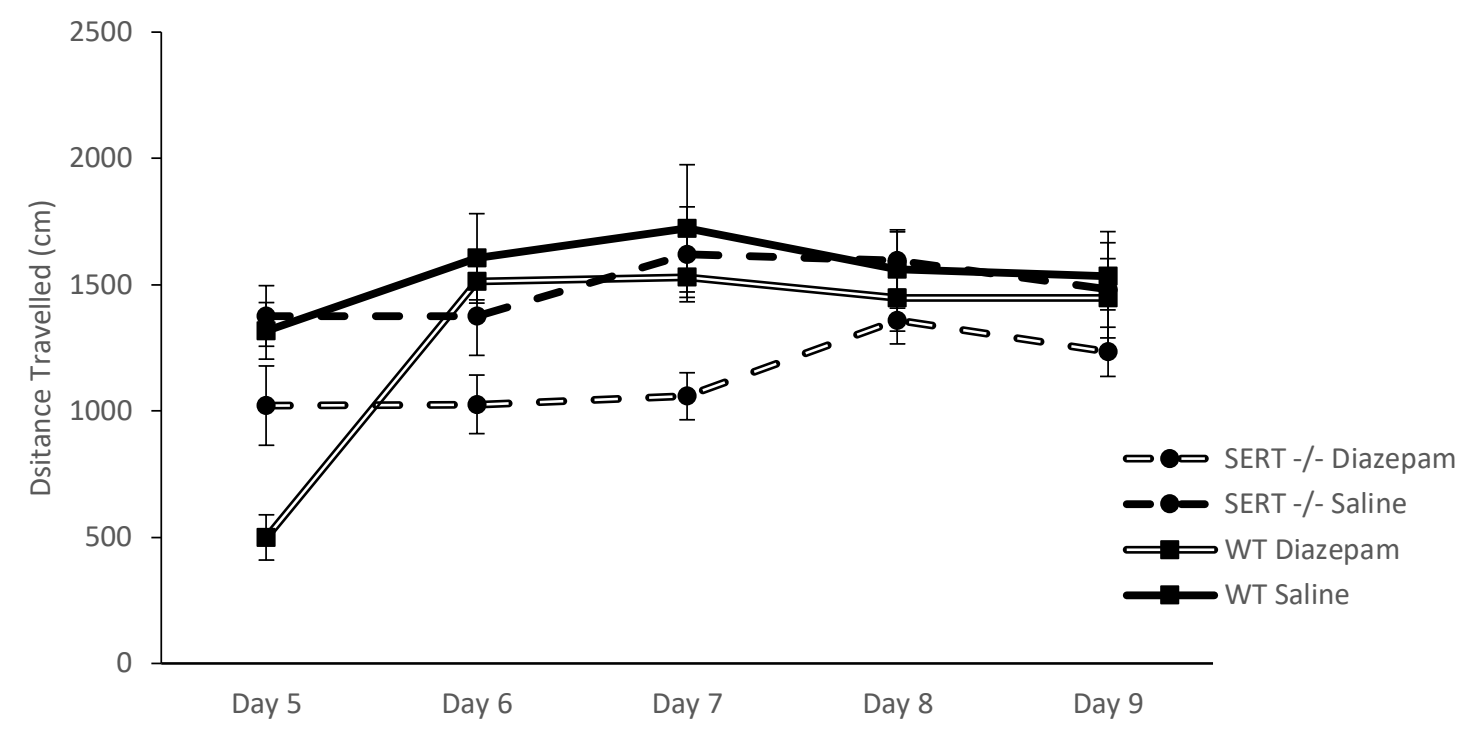

Figure 29. Means and \pm SEM of the long-term impact of diazepam on locomotor activity. Consecutive days are presented on the $x$ axis, and distance travelled in $\mathrm{cm}$ is depicted through the $y$ axis.

Ketamine treatment had no acute impact on locomotor activity (see table 10). However, a day*genotype interaction indicated that locomotor activity decreased from day 4 to 5 in the WT condition selectively (see appendix I). The long-term impact of ketamine revealed a main effect of day, whereby activity increased across the days of testing. A genotype*treatment interaction was also found, however planned comparisons found no difference between each treatment on each genotype (see appendix $I$ ). 
Table 10. Summary of Analyses on Locomotor Activity

\begin{tabular}{|c|c|c|c|}
\hline Analysis & Model Predictors & F Statistic & $\mathrm{p}$-value \\
\hline \multirow[t]{3}{*}{ Genotype Differences } & Day & $F(3,120)=16.23$ & $<.001 * * *$ \\
\hline & Genotype & $F(1,40)=0.03$ & .853 \\
\hline & Day*Genotype & $F(3,120)=0.40$ & .758 \\
\hline \multirow[t]{7}{*}{ Diazepam Acute } & Day & $F(1,28)=22.32$ & $<.001 * * *$ \\
\hline & Genotype & $F(1,28)=0.39$ & .537 \\
\hline & Treatment & $F(1,28)=17.59$ & $<.001 * * *$ \\
\hline & Day*Genotype & $F(1,28)=7.87$ & $.009 * *$ \\
\hline & Day*Treatment & $F(1,28)=11.00$ & $.003^{* *}$ \\
\hline & Genotype*Treatment & $F(1,28)=1.12$ & .299 \\
\hline & Day*Genotype*Treatment & $F(1,28)=6.07$ & $.020^{*}$ \\
\hline \multirow[t]{7}{*}{ Diazepam Long-Term } & Day & $F(2.88,66.34)=10.16$ & $<.001 * * *$ \\
\hline & Genotype & $F(1,23)=3.63$ & .069 \\
\hline & Treatment & $F(1,23)=10.12$ & $.004 * *$ \\
\hline & Day*Genotype & $F(2.88,66.34)=4.42$ & $.007 * *$ \\
\hline & Day*Treatment & $F(2.88,66.34)=2.39$ & .079 \\
\hline & Genotype*Treatment & $F(1,23)=0.30$ & .593 \\
\hline & Day*Genotype*Treatment & $F(2.88,66.34)=2.57$ & .064 \\
\hline \multirow[t]{7}{*}{ Ketamine Acute } & Day & $F(1,25)=1.34$ & .258 \\
\hline & Genotype & $F(1,25)=2.28$ & .144 \\
\hline & Treatment & $F(1,25)=0.00$ & .938 \\
\hline & Day*Genotype & $F(1,25)=4.67$ & $.040^{*}$ \\
\hline & Day*Treatment & $F(1,25)=0.23$ & .635 \\
\hline & Genotype*Treatment & $F(1,25)=3.56$ & .071 \\
\hline & Day*Genotype*Treatment & $F(1,25)=3.12$ & .090 \\
\hline \multirow[t]{7}{*}{ Ketamine Long-Term } & Day & $F(4,88)=6.81$ & $<.001 * * *$ \\
\hline & Genotype & $F(1,22)=0.05$ & .830 \\
\hline & Treatment & $F(4,88)=0.13$ & .722 \\
\hline & Day*Genotype & $F(4,88)=1.68$ & .161 \\
\hline & Day*Treatment & $F(4,88)=0.26$ & .906 \\
\hline & Genotype*Treatment & $F(1,22)=4.42$ & $.047 *$ \\
\hline & Day*Genotype*Treatment & $F(4,88)=1.08$ & .370 \\
\hline
\end{tabular}

Note. ${ }^{*} p<.05, * * p<.01, * * * p<.001$ 


\section{Discussion}

Experiment two aimed to assess the construct and predictive validity of the ADT by investigating the behavioural performance of rats with genetically reduced SERT activity, and the impact of anxiolytic and antidepressant treatments.

Overall, our hypothesis that the SERT $\%$ would show greater depression and anxietylike behaviour compared to WT was supported. In the anticipation box, WT subjects showed an anticipatory pleasure response, indexed by increased rearing across the days. This effect was not seen in the SERT $\%$ subjects, indicating reduced anticipatory pleasure, a depressionlike behaviour. No genotype differences were found in the total distance travelled in the anticipation box, indicating variations in rearing behaviour were not due to general differences in motor activity.

Interestingly, the proportion of $50 \mathrm{kHz}$ calls made increased across the days, and did not differ between genotypes, indicating both strains exhibited an anticipatory pleasure response. However, the genotype effect approached significance $(p=.088)$ and there was a large amount of individual variability which potentially obstructed this effect. In addition to simply the number of calls made, researchers have detected at least 14 'subtypes' of calls within this $50 \mathrm{kHz}$ range, each with distinct patterns of frequency modulation, suggesting the morphological properties of USVs may also encode affective meaning (Brudzynski, 2005). During social play anticipation, specific subtypes of $50 \mathrm{kHz}$ vocalisations were found to be strongly associated with distinct behaviours (Burke et al., 2017). Furthermore, research which found no differences in the number of $50 \mathrm{kHz}$ calls made during social interaction between strains of rats, noted significant differences in other parameters of the calls (Houwing et al., 2019). These findings indicate that investigation of the morphological parameters of USVs may offer additional information beyond what investigation of quantity provides. Unfortunately, time constraints prevented this type of analysis, but future analysis of the USV recordings may identify genotype differences in call type.

In the successive alleys part of the test, SERT \% subjects exhibited increased anxietylike behaviour indexed by less time spent in anxiogenic alleys ( 3 and 4$)$, and lower retrieval scores, compared to WT subjects. No genotype differences were found in retrieval latency, indicating differences in retrieval score were not due to varying motivation to consume the food reward. These findings are in line with previous literature which reports increased depressive and anxiety-like behaviour in SERT $\%$ subjects (Mohammad et al., 2016; Olivier et 
al., 2008). Importantly, our data shows that the symptoms are stable over time and can be repeatedly assessed.

As the serotonergic system has been widely implicated in human psychiatric disorders (Serretti et al., 2007), the impact of variations to this system are crucial to consider in an animal model of such disorders. Human studies have shown the short allelic variation of the 5HTTLPR polymorphism reduces expression of the SERT and has been commonly associated with increased risk of depression and anxiety diagnosis (Karg et al., 2011; Mazzanti et al., 1998). Genetic manipulation was employed to create the SERT knockout rodent model, which results in subjects with varying SERT levels seen in the human condition. Given the wellestablished link between SERT reduction in humans and increased risk for depression and anxiety disorders, the SERT model is a useful tool for assessing construct validity. However, the SERT $\%$ rat exhibits a complete absence of SERT, therefore it is limited in its ability to mimic the human condition in its entirety, as SERT expression is only partially reduced in humans expressing the short allelic variation of the 5-HTTLPR polymorphism (Nakamura et al., 2000). Additionally, a gene $\mathrm{x}$ environment interaction whereby exposure to a stressor greatly increases risk of a depression and/or anxiety disorder in humans with reduced SERT is well established (Houwing et al., 2017), yet the SERT $\%$ display an anxious and depressive phenotype in the absence of a stressor.

The homozygous SERT ${ }^{\prime}$ genotype was selected over the heterozygous SERT ${ }^{+/}$which better aligns with the human condition, displaying a 50\% reduction in SERT, as previous research failed to detect differences between SERT ${ }^{+}{ }^{-}$and WT subjects on multiple measures of the ADT (Kidwell, 2019). The discrepancy between the SERT $\%$ and human condition denotes that an imperative avenue to further validate the construct validity of the ADT is the assessment of the SERT $+/$ genotype exposed to a stress induction. However, the current study employed a more optimized version of the ADT than Kidwell (2019), therefore investigation of the SERT $+/$ exclusive of environmental manipulation, utilizing the current protocol is also advised.

Stressful experiences are widely recognized for the role they play in the pathogenesis of depressive and anxiety disorders in clinical samples (McCormick \& Green, 2013). Accordingly, numerous stress procedures have been utilized in animal models of these disorders. A commonly used procedure is the chronic unpredictable stress paradigm, however the duration of exposure to stressors and stressors used vary across literature. Typically, a combination of physical (restraint, damp bedding, cage tilt), predatory (urine, fur, vocalizations from cats or foxes), and social (isolation, crowding, novel cage mates) stressors are presented 
to subjects over the course of 2-8 weeks. This form of stress protocol has been shown to increase both depressive and anxiety-like behaviours across a range of tests (Kopradova et al., 2018; Sequeira-Cordero et al., 2019; Yohn \& Blendy, 2017), and leads to adaptations in key brain regions which align with changes we see in the human population (McCormick \& Green, 2013). Animal models of stress appear to be advantageous and should be investigated in future to develop on the current findings and construct validity of the ADT.

Additionally, clinical studies on humans have highlighted the significant role early childhood trauma, such as social separation and abuse, has on the development of an affective disorder later in life (Freund et al., 2013). Accordingly, animal models of these disorders have employed manipulations such as maternal separation (removing pups from the dam) to induce an anxious and depressive-like phenotype. This model has further been neuroanatomically validated, with rodents' subject to early maternal separation showing reductions in GABAeric markers such as parvalbumin, a finding which parallels the reduced GABA activity seen in adolescents with depression (Leussis et al., 2012). Numerous studies have found that early maternal separation increases the amount of time adult rats spend immobile in the FST (Cui et al., 2020; Kwak et al., 2009; Marais et al., 2008), and reduces the number of entries and time spent in open arms of the EPM (Wang et al., 2020), indicating this model may be a valuable tool for supplementary research on the ADT.

Providing further support for the use of the ADT, the present study showed that pharmacological treatments improved performance on the relevant aspects of the test, supporting the predictive validity of the ADT. On days $1-4$ the SERT $\%$ subjects showed a deficit in anticipatory pleasure indexed by less rearing behaviour compared to WT. After a single administration of ketamine, SERT \% subjects showed an increase in rearing behaviour compared with SERT $\%$ controls. This effect was selective to the SERT $\%$ strain as no difference was found between treatment conditions for WT subjects. Furthermore, following ketamine treatment, SERT $\%$ rearing was equivalent to WT subjects, indicating a normalization of behaviour. Ketamine treatment had no impact on distance travelled in the anticipation box, indicating changes to rearing behaviour were not due to general locomotor effects. This effect was still seen at day 9 of testing, indicating the impact of a single ketamine treatment lasted at least 4 days. These findings align with clinical research which indicates a single subanaesthetic administration of ketamine improves self-reported depressive symptoms for between $1-4$ weeks (Ibrahim et al., 2012; Zarate et al., 2006). The longevity of symptom improvement after acute ketamine administration is less consistent in animal research. Antidepressant effects were seen in the FST 2 months after twice daily administration of $20 \mathrm{mg} / \mathrm{kg}$ for 15 days, however 
this effect was not found after a single administration (Parise et al., 2013). Chen and colleagues (2021) report a single $10 \mathrm{mg} / \mathrm{kg}$ dose increased sucrose preference, 1 and 3 days after administration, however this effect was not found 11 days after treatment. In mice, a single dose of $10 \mathrm{mg} / \mathrm{kg}$ produced anti-depressant effects in the sucrose preference test 8 days after administration (Ma et al., 2013), and FST and tail suspension test 30 days after administration (Viana, 2021). In contrast, one week after a single dose of either $2.5,12.5$ or $40 \mathrm{mg} / \mathrm{kg}$, no behavioural effects were found in the FST (Bechtholt-Gomf, 2011).

Further research is required to evaluate whether ketamine treated SERT $\%$ subjects behaviour did not return to baseline due to long-lasting treatment effects, or whether it is a feature of the test. However, as discussed in detail later in the chapter, the effect of diazepam was acute, indicating not all treatment effects are long lived. The longevity of symptom improvement after treatment is crucial to assess as it has significant implications for the future use of the ADT. If behaviour returns to baseline after treatment, this will allow subsequent research to employ the ADT to investigate the duration of treatment efficacy after cessation, and investigate multiple treatment options in the same subject, having widespread clinical applications.

Ketamine had no impact on the proportion of 50kHz USVs emitted, a finding which does not support our hypothesis that ketamine would alleviate anhedonia, when indexed by $50 \mathrm{kHz}$ USVs. Whilst this finding was not expected, previous literature investigating the impact of ketamine on USV emission is limited, however appears to be in line with the current findings. Popik and colleagues (2017) demonstrated that ketamine reduced immobility on the FST, however it also decreased the number of 50kHz USVs emitted during social interaction. Similar results have been observed with other NMDA receptor antagonists. Acute administration of phencyclidine and MK-801 to juvenile rats inhibited tickling induced $50 \mathrm{kHz}$ USVs (Wendler et al., 2016), and MK-801 reduced 50kHz USVs when exposed to an amphetamine-paired environment (Costa et al., 2015). These findings suggest the antidepressant effects of ketamine may not be captured via USVs. As mentioned earlier, investigation of call subtypes may also be applied to provide further insight into affective state.

Our results also indicate ketamine treatment had no effect on measures of anxiety-like behaviour, such as latency to enter and duration spent in anxiogenic alleys, and retrieval score. Previous research investigating the anxiolytic effects of ketamine is conflicting across human and animal research. A single administration of $10 \mathrm{mg} / \mathrm{kg}$ had no effect on behaviour in the EPM, whereas $50 \mathrm{mg} / \mathrm{kg}$ increased entries to open arms, suggesting an anxiolytic effect (Engin et al., 2009). Conversely, Silvestre et al. (1997), found $7 \mathrm{mg} / \mathrm{kg}$ decreased duration spent and 
entries to open arms in the EPM, indicating an anxiogenic effect. This incongruence may be due to dose effects, as the current study employed a lower dose than those previously mentioned. In clinical studies, Glue and colleagues (2020) report a single treatment of either $0.25,0.5$ or $1 \mathrm{mg} / \mathrm{kg}$ decreased self-reported anxiety within 1 hour, with effects persisting up to 1 week. Whereas a placebo-controlled study found no difference in self-reported anxiety between ketamine treatment $(0.5 \mathrm{mg} / \mathrm{kg})$ and placebo across 14 days (Taylor et al., 2018). As the literature is inconsistent, our findings add to existing literature on the anxiolytic effects of ketamine. In addition, these results also support the dissociation of measures within the test, i.e. behaviour in the anticipation box as an index of anhedonia, and behaviour in the alley as an index of anxiety, further supporting the predictive validity of the ADT.

In line with our hypothesis and providing additional support for the predictive validity of the ADT, diazepam treatment induced an anxiolytic effect in the successive alleys test. However, unlike ketamine, this treatment effect was not selective for the SERT $\%$ subjects. On the day of administration, diazepam significantly increased retrieval scores, and duration spent in alley 3, compared to controls. This effect was not found on the following days of testing, indicating behaviour returned to baseline within 24 hours of treatment. These findings also support our hypothesis that the ADT does not suffer from the one-trial tolerance effect and possesses the ability to test multiple pharmacological treatments in the same animal. Subsequent research is required to substantiate this conclusion. After subjects have returned to baseline following initial treatment, an additional treatment could be given to determine whether a second treatment is also effective, and if behaviour returns to baseline once again.

Interestingly, diazepam did not impact the duration spent in alley 4 on the day of administration, although a significant three-way interaction was found when analysing the long-term impact of treatment. As depicted in Figure 19, this interaction appears to be primarily driven by behaviour in the diazepam treated WT condition, which showed a rather erratic pattern. Despite planned comparisons indicating no differences between saline and diazepam treatment, this pattern of behaviour is peculiar, limiting the conclusions drawn from this interaction result. Further investigation removed outliers $( \pm 2 S D)$ from the analysis, however the pattern persisted. One possible explanation is that as subjects spent more time in this alley, they began to lean/look over the edge. Whilst the trial where the subject completely fell off the alley was removed from the data, in some cases a subject would be leaning over the edge, almost fall, but manage to clamber back up onto the apparatus without assistance. This near miss may have frightened subjects, causing them to be more wary on the subsequent trial. 
However, this explanation is based purely off retrospective consideration, and requires replication to better understand this finding.

Despite not finding a treatment effect of diazepam on some measures of anxiety, such as the duration in alley 4 and latency to anxiogenic alleys, the significant treatment effect on other measures of anxiety-like behaviour nonetheless provides preliminary support for the predictive validity of the test. Our results also indicate the ADT does not suffer from the onetrial tolerance, however the assessment of treatment on earlier days of testing (i.e. day 1) may provide further insight to this proposition. If prior to multiple exposure, diazepam shows anxiolytic effects on measures which were not seen on day 5, this may suggest some form of trial tolerance.

Diazepam also selectively decreased the proportion of $50 \mathrm{kHz}$ USVs made and rearing behaviour on day 5 in WT subjects. Whilst virtually no previous literature suggests diazepam reduces anticipatory pleasure, the sedative and muscle-relaxant effects are widely recognised (File \& Fernandes, 1994). The significant decrease in distance travelled in the anticipation box in this group, further suggests changes to USVs and rearing are likely to be due to the sedative effect of treatment, as opposed to an alteration in anticipatory pleasure.

Overall, the results of the current study provide preliminary support for the predictive validity of the ADT, however further research is necessary to substantiate these findings. Ketamine was selected for the current study as a majority of established treatments fail to demonstrate efficacy after acute treatment, and they operate on the serotonergic system, therefore were inoperable on the selected animal model (SERT $\%$ ). However, the SERT $\%$ model may be relevant for investigating further treatment options for patients who do not respond to SSRIs. Clinical research has found that ketamine effectively improves depression symptoms in treatment resistant patients (Zarate et al., 2006), a finding which parallels the improvements we found in the SERT $\%$.

Despite numerous studies highlighting the antidepressant effects of ketamine, the mechanism via which this NMDA antagonist produces these effects remains unknown, warranting further investigation of well-established antidepressants. Furthermore, the mechanism of action varies amongst established antidepressants and anxiolytic treatments, therefore investigation of a tests sensitivity to a range of treatments is imperative. The FST captures the effects of a broad range of antidepressants such as norepinephrine reuptake inhibitors, selective serotonin reuptake inhibitors, reversible inhibitors of monoamine oxidase, and tricyclic antidepressants (Cryan \& Lucki, 2000; Cryan et al., 2005). Similarly, the EPM shows sensitivity to a variety of anxiolytic treatments including both long (e.g. diazepam and 
chlordiazepoxide) and short (e.g. lorazepam) lasting benzodiazepines, barbiturates, beta blockers, and sedative-hypnotics (Audi et al., 1989; Garcia et al., 2011; Mar et al., 2002; Schmitt \& Hiemke, 1998). Furthermore, anxiogenic compounds such as yohimbine, caffeine and amphetamine, increase anxiety-like behaviour in the EPM (Pellow et al., 1985). The expanse of these findings reinforces the predictive validity of these tests and their widespread use. To align the ADT with these established tests, and ensure the test is reliable and valid, investigation of treatment effects across a variety of drug classes is crucial and should be executed in future studies.

A second component of predictive validity is the convergence of treatment conditions between clinical and animal research. The impact of a treatment such as fluoxetine, which has been widely established to show efficacy after chronic but not acute administration in humans (Nierenberg et al., 2000), should be investigated in the ADT to establish whether these conditions align, further supporting the predictive validity of the test.

Seeing that the ADT incorporates food restriction, future research is required to determine the potential impact this may have on the tests ability to detect antidepressant efficacy. In line with our findings, previous literature found the antidepressant effects of ketamine remained intact when subjects were food restricted (Ecevitoglu et al., 2019). However, as discussed in chapter 2, food restriction has been shown to reduce the effects of traditional antidepressants (Soubrié et al.,1988), therefore merits consideration. 


\section{Chapter Four}

\section{Discussion of the Current Findings and Their Applications to Human and Animal Research}


Animal models are designed to represent characteristics of the human condition, ranging from disease and illness (Hau, 2008), to cognitive and emotional processes (Jonasson, 2005; Paul et al., 2005), with over 100 rodent behavioural tests being used currently (Belovica et al., 2017). Many of these tests are designed with the intent of measuring depression and anxiety-like behaviours and are employed to gain further understanding of the underpinnings and effects of these disorders, and screen potential pharmacological treatments. However, as outlined in chapter 1, current animal tests of these disorders display numerous shortcomings which hinder the applicability of the observed behaviours to the human condition. These limitations likely contribute to the lack of novel pharmacological treatments which display efficacy in human trials, and high rates of treatment-resistant patients (Planchez et al., 2019).

The current thesis aimed to develop a novel test of depressive and anxiety-like behaviour in rodents, which addresses previous tests limitations. A recap of all findings can be found in Table 11.

In the first experiment, food restricted subjects displayed substantially greater anticipatory responses, indexed by increases in rearing behaviour and total number of $50 \mathrm{kHz}$ calls emitted. These results imply that despite frootloops being a palatable food, food restriction is required to increase the value of this reward enough to create an adequate anticipatory pleasure response, supporting the use of food restriction when employing the ADT. Food restriction had no impact on latency to enter, or duration spent in anxiogenic alleys, however, did increase retrieval scores. Given that retrieval scores were found to be the most sensitive measure of anxiety-like behaviour (Kidwell, 2019), we conclude that food restriction is required in order to capture a full range of scores and avoid possible floor effects. Limitations of food restriction are discussed in chapter 2, and we encourage researchers to consider these implications in future use of the ADT.

The key findings of this thesis are that [1] diazepam treatment increased the duration spent in alley 3 and retrieval scores, implying an anxiolytic effect, [2] ketamine increased rearing behaviour in the SERT $\%$ condition, indicating a selective antidepressant effect, and [3] SERT $\%$ exhibited anhedonia indexed via decreased rearing behaviour, and increased anxietylike behaviour indexed via decreased duration in anxiogenic alleys and lower retrieval scores.

These results align with previous research on the effects of these treatments (Carrier \& Kabbai, 2013; Wilson et al., 2004), and behavioural patterns of SERT $\%$ rats (Olivier et al., 2008) and mice (Lira et al., 2003; Sakakibara et al., 2014), providing preliminary support for the use of the ADT as a valid test to assess depression and anxiety-like behaviour in rodents. 
Future directions to supplement the construct and predictive validity have been discussed in chapter 3.

Construct validity denotes animal models have theoretical comparisons to the human disorder, and predictive validity implies treatment efficacy is equivalent between animal models and clinical samples. Therefore, establishing the ADT displays construct and predictive validity, suggests findings from animal models in this test will be applicable to the human condition, improving the translatability between animal and human research.

Furthermore, this thesis demonstrates the ADT circumvents limitations of previous tests of depression and anxiety-like behaviour in rats, such as the one-trial-tolerance and inability to investigate these disorders comorbidly. Utilizing anticipatory pleasure as an index of anhedonia offers a more valid measure of depression-like behaviour than established tests, which improves translatability to human behaviour. As discussed subsequently, the elimination of these limitations has widespread applicability to both animal and human research. 
Table 11. Recap of Findings

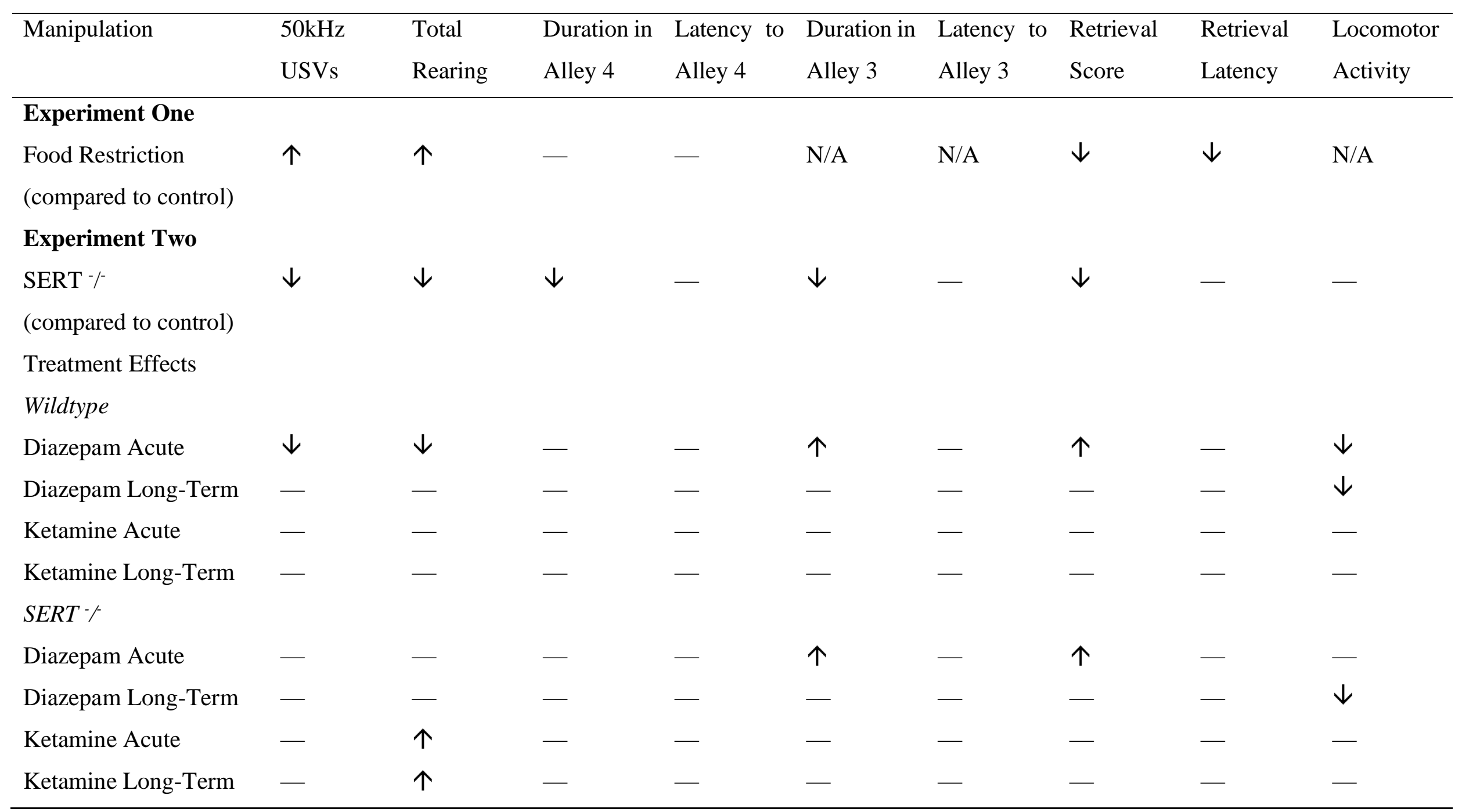




\section{Advantages and Applications of the ADT}

The creation of a test which does not suffer from the one-trial-tolerance is a significant development in animal research. Continuous improvements are being made to the welfare of animals used in science, with an explicit set of principles known as the "Three R's" being applied to guide ethical evaluation of research (Kirk, 2018). The elimination of the one-trialtolerance is specifically relevant for the "reduce" principle. The aim of this tenet is a push toward strategies which result in sufficient data being gathered from as few subjects as possible, or the maximization of information obtained per animal, subsequently reducing the use of additional subjects. The potential ability to test multiple treatment options in the same animal will greatly reduce the number of subjects required to attain equivalent results, fulfilling the reduce principle. Diazepam administration did not appear to result in long-term alterations to behaviour, as on the following days of testing, the treated subject's behaviour aligned with the control subjects. These findings indicate subject's behaviour returns to baseline after initial treatment, possibly allowing the assessment of additional treatment options in the same animal. Future directions to substantiate the ability to investigate multiple treatments in the same subject using the ADT have been discussed in chapter 3.

Furthermore, to investigate treatment efficacy, an anxious phenotype is often induced via manipulations such as maternal separation or unpredictable chronic stress. Reducing the number of subjects required, will in turn require fewer subjects to endure these manipulations, reducing the overall harm involved.

In addition to ethical benefits, repeated testing allows investigations concerning duration of therapeutic efficacy. The findings of experiment two indicate the antidepressant effect of ketamine lasted at least 4 days, whereas the anxiolytic effect of diazepam was absent 24 hours later. The short duration of the effects of diazepam, indicates frequent administration is required to consistently achieve anxiolytic action. This short-term treatment efficacy is an obstacle in clinical practice, as studies indicate repeated administration of drugs such as benzodiazepines results in tolerance to the effects of this treatment (Stephens \& Schneider, 1985). Investigating the duration of treatment efficacy may assist in advancements in understanding and establishing treatments which have longer-lasting effects, therefore reducing administration requirements and subsequent tolerance development. Additionally, repeated testing utilizing the $\mathrm{ADT}$ allows for precise evaluation of the duration to onset of efficacy of treatment, and the duration of efficacy following varying treatment periods. These investigations will facilitate the establishment of clinical treatment protocols, such as 
generating alternative treatment options to decrease risk to the patient during this period of delayed onset of typical antidepressants, as well as further insight to the minimally effective duration of treatment to reduce the risk of relapse after discontinuation.

A final benefit of repeated testing of anxiety-like behaviour in animal models is the ability to investigate the long-lasting facet of anxiety which is often found in clinical patients. Anxiety in humans is commonly conceptualized in two forms, state and trait anxiety. State anxiety is defined as a transient emotional response to an environmental stressor, involving physiological arousal and feelings of apprehension and worry. In contrast, trait anxiety is seen as an enduring personality characteristic which predisposes an individual to present state anxiety (Kennedy et al., 2001). These two dimensions of anxiety show psychometric independence in clinical studies and dissociative impacts on cognitive processes such as attention (Lau et al., 2006; Pacheco-Unguetti et al., 2010). Distinctions have also been made between the aetiological basis of each state. State anxiety was found to be primarily influenced by environmental factors, whereas environmental and genetic factors contributed similarly to trait anxiety (Lau et al., 2006; Legrand et al., 1999).

As current tests of anxiety-like behaviour in rodents suffer from the one-trial-tolerance, anxiety-like behaviour is only able to be assessed at a single time point, therefore preventing this research from differentiating between the two forms. Many animal models employ environmental manipulations (e.g. unpredictable chronic stress) to induce an anxious phenotype. Based off the findings that environmental and genetic factors impact trait anxiety equivalently, a purely environmental manipulation may be producing a phenotype which corresponds more to state anxiety. Consequently, the development of treatments is being guided by research on this form of anxiety, not capturing or attending to the trait facet of anxiety. As the ADT has the ability to detect differences between short and long-lasting anxiety-like behaviour, utilizing this test will contribute to the disentanglement of these dimensions, including biologically and environmentally mediated pathways, in turn advancing our understanding of this disorder and treatment methods.

The comorbidity of anxiety and depressive disorders is exceedingly common and is receiving increasing attention in clinical research. Patients who present with comorbid depressive and anxiety disorders display more severe symptoms, increased risk of suicidal ideation and behaviour, poorer responses to treatment, and greater risk of remission compared to those who present the disorder in isolation (Mathew et al., 2011).

The ability to investigate depression and anxiety-like behaviour when it presents comorbidly is vital for developing appropriate treatments. Demonstrating efficacy in each 
disorder when presented individually is not to say this treatment is equally effective when the disorders occur together. Despite evidence suggesting imipramine is an effective treatment for both major depression and panic disorder, when assessed in patients presenting these disorders comorbidly, treatment improved major depression symptoms, yet failed to have an effect on the number or type of panic attacks experienced (Keller et al., 1993).

Additional considerations such as dose requirement, duration until onset of efficacy, and minimally effective duration may also differ when disorders present together compared to in isolation. Furthermore, greater understanding is required surrounding the lack of consistency between responses to treatment for each of the disorders, and prospective treatment options for patients when only one of the disorders has responded.

In order to parallel human psychopathology as it presents in clinical samples, animal models must undergo continuous conceptual refinement. To our knowledge, the ADT is the only test which allows for the assessment of comorbid depression and anxiety-like behaviour in animals, providing the field with an essential and innovative research tool.

With the establishment of a test with the ability to measure comorbid depression and anxiety, an important direction for future studies is utilizing and further developing specialized animal models which display comorbid symptoms. This thesis provides support for the use of the SERT knockout rat as an animal model of these disorders comorbidly, as the knockout (SERT \%) strain displayed increased levels of anxiety and anhedonia compared to wildtype controls. However, the use of this model is limited due to the absence of the serotonin transporter in the homozygous knockout (SERT $\%$ ) strain.

Selective serotonin reuptake inhibitors, the most commonly prescribed treatment for both major depression and anxiety disorders (Belzer \& Schneier, 2004), increase extracellular serotonin concentrations by inhibiting the serotonin transporter, a mechanism of action which is rendered ineffective in the SERT $\%$ model. Whilst the efficacy of other popular treatments such as serotonin-norepinephrine reuptake inhibitors (SNRIs) on behaviour in the SERT $\%$ has not been established, this model shows neuroplastic changes in response to chronic duloxetine treatment (Calabrese et al., 2010). Therefore it may be suitable for investigating the effects of this treatment class on behavioural measures.

As outlined in chapter 3, the SERT $\%$ model may also provide a useful model for investigating novel interventions for treatment resistant patients. Furthermore, depression and anxiety in humans is associated with a reduction, not complete absence of SERT (Nakamura et al., 2000), and a gene $\mathrm{x}$ environment interaction, therefore an evident next step is 
investigation of an environmental stressor on the SERT ${ }^{+}{ }^{-}$genotype which show a comparable $50 \%$ reduction in SERT (Homberg et al., 2007).

To improve the translatability of animal research into clinical application, modelling comorbidity in animal models presents an area of significant relevance. The ADT may be employed in future as a tool for validating novel animal models of these disorders, offering advancements in an important step for understanding and creating treatments for comorbid disorders in clinical populations.

\section{Considerations and Future Directions}

When employing the ADT, the floor effect found in duration spent in alley 4 in experiment two should be considered. Only $30 \%$ of subjects in the second experiment entered this alley on the first day of testing, compared with $60 \%$ of subjects in experiment one. This discrepancy may be due to the shift from Sprague-Dawley rats to Wistar, as the former strain typically shows a less anxious profile compared to the latter (Rex et al., 2004). As handling of subjects is also recognised to impact rats' anxiety-like behaviour, alterations to this procedure may also account for the considerable decrease found. In experiment one, subjects were handled for 5 days prior to testing, during the habituation period to the anticipation box. When the habituation period was adjusted to 3 days for experiment one, handling of subjects was also decreased to 3 days. Although handling was only reduced by 2 days, this in combination with utilizing a strain which is inherently more anxious may have resulted in this floor effect. As the aim of the ADT is to investigate anxiety-like behaviour, we recommend future research adapts the protocol to include a minimum of 5 days of handling prior to testing.

The opposing impact of extended handling must also be considered due to the repeated nature of testing. Anxiety-like behaviour in both the elevated plus maze and open field test was reduced in subjects handled for 5 minutes daily for seven days prior to testing, compared to unhandled subjects (Schmitt \& Hiemke, 1998), indicating handling has an anxiolytic effect. As mentioned above, acute handling may be beneficial in order to reduce floor effects, however the ability to detect varying anxiety levels between experimental conditions may be impacted by chronic handling leading to a normalization of anxiety-like behaviour. In addition, anxiolytic effects of diazepam fail to be seen in subjects exposed to 28 days of handling (Brett \& Pratt, 1990), a finding which implicates the ADT protocol for assessing multiple treatment options. Employing the ADT in future to investigate multiple treatments will involve prolonged 
testing periods, therefore caution should be taken to reduce animal handling to ensure treatment effects are not diminished due to handling habituation.

A final limitation is the possibility of experimenter bias on manually recorded measures such as rearing behaviour. Due to the nature of the study, blinding the experimenter to subject condition was unfeasible. Calculating inter-rater reliability was also prevented due to time and resource constraints, however, should be considered in future to ensure the reliability of this measure. An alternative solution is to utilize behavioural tracking software such as Ethovision. This would require the use of a second recording camera to ensure visibility into the anticipation box was sufficient to capture these movements. Similarly, due to limited resources this was not feasible for the current study.

As this thesis focuses on the development of a novel test, qualitative observations should also be noted. During the last days of testing, subjects would occasionally climb onto the walls of alley 1 . This behaviour appears reflective of a lack of anxiety, however, is recorded as duration spent in alley 1 , indexing anxiety-like behaviour. The experimental data shows a linear decrease in duration spent in this alley across days of testing (data not shown), therefore this behaviour does not appear to have impacted results, however, warrants attention in future.

Also towards the final days of testing, it was observed that a few subjects frequently jumped within the anticipation box. Jumping behaviour has been observed in rats during play (Kisko, Wohr, Pellis \& Pellis, 2017), and after tickling (Ishiyama, Kaufmann \& Brecht, 2019). Furthermore, animal models of depression show reduced jumping behaviour during anticipation of play (Burke et al., 2012), indicating jumping may reflect positive affect. Based off this previous literature and observations of the current study, jumping behaviour may provide an additional measure of anticipatory pleasure and should be considered in future studies.

A critical direction for future research is the incorporation of female subjects. The prevalence of major depression and anxiety disorders is twice as high in females compared to males (LeGates et al., 2019), yet approximately 90\% of animal research on depression and anxiety-like behaviours has utilized exclusively male subjects (Fuchs \& Flügge, 2006), creating a substantial disconnect between clinical findings and animal research. Age of onset, severity and presentation of symptoms, response to treatment, and duration of episodes is also sexually dimorphic in clinical patients (LeGates et al., 2019), further highlighting the importance of investigating sex differences in animal models. Currently, research on sex differences in the expression of depression and anxiety-like behaviour in rats is widely inconsistent, with findings varying between studies and across measures (Knight et al., 2021; 
Ma et al., 2019). However, literature is in agreement that sex differences in factors such as neurochemical, hormonal and biological differences between sexes are associated with the variance in precipitation, presentation, and response to treatment of these disorders (Dalla et al., 2009). Limiting research surrounding depression and anxiety to male subjects reduces the translatability of findings to female populations, hindering advancements in our understanding of these disorders and treatment options.

In summary, the preceding chapters investigated a novel test of depression and anxietylike behaviour in rats, with the intent of establishing a comprehensive protocol and assessing construct and predictive validity. The benefits of the ADT, limitations of the current study, and directions for future use of this test have also been discussed. Our findings highlight the ADT as an innovative and valuable tool to measure depression and anxiety-like behaviour in rats, which provides the field with the ability to notably advance current understanding of these disorders and improve treatment options. 


\section{References}

Al-Harbi, K. S. (2012). Treatment-resistant depression: Therapeutic trends, challenges, and future directions. Patient Preference and Adherence, 6, 369-388.

Aseltine, R. H., Gore, S., \& Colten, M. E. (1998). The co-occurrence of depression and substance abuse in late adolescence. Development and Psychopathology, 10(3), 549570.

Assis, L. C., Rezin, G. T., Comim, C. M., Valvassori, S. S., Jeremias, I. C., Zugno, A. I., ... \& Streck, E. L. (2009). Effect of acute administration of ketamine and imipramine on creatine kinase activity in the brain of rats. Brazilian Journal of Psychiatry, 31(3), 247252.

Audi, E. A., De-Oliveira, C. E., \& Graeff, F. G. (1989). Serotonergic mediation of the anxiolytic effect of intracerebrally injected propranolol measured in the elevated plusmaze. Brazilian journal of medical and biological research, 22(6), 699-701.

Bahi, A., Schwed, J. S., Walter, M., Stark, H., \& Sadek, B. (2014). Anxiolytic and antidepressant-like activities of the novel and potent non-imidazole histamine H3 receptor antagonist ST-1283. Drug design, development and therapy, 8, 627.

Ballenger, J. C. (2000). Anxiety and Depression: Optimizing Treatments. Primary Care Companion to The Journal of Clinical Psychiatry, 2(3), 71-79.

Baxter, A. J., Vos, T., Scott, K. M., Ferrari, A. J., \& Whiteford, H. A. (2014). The global burden of anxiety disorders in 2010. Psychological medicine, 44(11), 2363.

Bechtholt-Gompf, A. J., Smith, K. L., John, C. S., Kang, H. H., Carlezon, W. A., Cohen, B. M., \& Öngür, D. (2011). CD-1 and Balb/cJ mice do not show enduring antidepressantlike effects of ketamine in tests of acute antidepressant efficacy. Psychopharmacology, 215(4), 689-695.

Beck, A. T., Alford, B. A., Beck, M. A. T., \& Alford, P. D. B. A. (2014). Depression. University of Pennsylvania Press.

Belovicova, K., Bogi, E., Csatlosova, K., \& Dubovicky, M. (2017). Animal tests for anxietylike and depression-like behavior in rats. Interdisciplinary Toxicology, 10(1), 40-43.

Belzer, K., \& Schneier, F. R. (2004). Comorbidity of Anxiety and Depressive Disorders: Issues in Conceptualization, Assessment, and Treatment: Journal of Psychiatric Practice, $10(5), 296-306$. 
Bodnoff, S. R., Suranyi-Cadotte, B., Aitken, D. H., Quirion, R., \& Meaney, M. J. (1988). The effects of chronic antidepressant treatment in an animal model of anxiety. Psychopharmacology, 95(3), 298-302.

Brett, R. R., \& Pratt, J. A. (1990). Chronic handling modifies the anxiolytic effect of diazepam in the elevated plus-maze. European journal of pharmacology, 178(1), 135-138.

Brotto, L. A., Gorzalka, B. B., \& Barr, A. M. (2001). Paradoxical effects of chronic corticosterone on forced swim behaviours in aged male and female rats. European journal of pharmacology, 424(3), 203-209.

Brudzynski, S. M. (2005). Principles of Rat Communication: Quantitative Parameters of Ultrasonic Calls in Rats. Behavior Genetics, 35(1), 85-92.

Buck, C. L., Malavar, J. C., George, O., Koob, G. F., \& Vendruscolo, L. F. (2014). Anticipatory $50 \mathrm{kHz}$ ultrasonic vocalizations are associated with escalated alcohol intake in dependent rats. Behavioural Brain Research, 271, 171-176.

Burke, C. J., Kisko, T. M., Swiftwolfe, H., Pellis, S. M., \& Euston, D. R. (2017). Specific 50$\mathrm{kHz}$ vocalizations are tightly linked to particular types of behavior in juvenile rats anticipating play. PLOS ONE, 12(5), e0175841.

Burke, C. J., Modlinska, K., Mauro, M. H., Aleksandrova, L. R., Pellis, S. M., Phillips, A. G., \& Euston, D. R. (2021). A naturalistic method to test depression: Anticipation of play. Behavioural Brain Research, 398, 112975.

Cadoni, C., Solinas, M., Valentini, V., \& Chiara, G. D. (2003). Selective psychostimulant sensitization by food restriction: Differential changes in accumbens shell and core dopamine. European Journal of Neuroscience, 18(8), 2326-2334.

Calabrese, F., Molteni, R., Cattaneo, A., Macchi, F., Racagni, G., Gennarelli, M., Ellenbroek, B. A., \& Riva, M. A. (2010). Long-Term Duloxetine Treatment Normalizes Altered Brain-Derived Neurotrophic Factor Expression in Serotonin Transporter Knockout Rats through the Modulation of Specific Neurotrophin Isoforms. Molecular Pharmacology, 77(5), 846-853.

Carrier, N., \& Kabbaj, M. (2013). Sex differences in the antidepressant-like effects of ketamine. Neuropharmacology, 70, 27-34.

Chaouloff, F., Durand, M., \& Mormède, P. (1997). Anxiety-and activity-related effects of diazepam and chlordiazepoxide in the rat light/dark and dark/light tests. Behavioural brain research, 85(1), 27-35.

Chen, L., Ke, Y., Ma, H., Gao, L., Zhou, Y., Zhu, H., Liu, H., Zhang, F., \& Zhou, W. (2021). Fluoxetine and Ketamine Reverse the Depressive but Not Anxiety Behavior Induced 
by Lesion of Cholinergic Neurons in the Horizontal Limb of the Diagonal Band of Broca in Male Rat. Frontiers in Behavioral Neuroscience, 15.

Commons, K. G., Cholanians, A. B., Babb, J. A., \& Ehlinger, D. G. (2017). The Rodent Forced Swim Test Measures Stress-Coping Strategy, Not Depression-like Behavior. ACS Chemical Neuroscience, 8(5), 955-960.

Costa, G., Morelli, M., \& Simola, N. (2015). Involvement of glutamate NMDA receptors in the acute, long-term, and conditioned effects of amphetamine on rat $50 \mathrm{kHz}$ ultrasonic vocalizations. International Journal of Neuropsychopharmacology, 18(11), 1-12.

Cryan, J. F., \& Lucki, I. (2000). Antidepressant-like behavioral effects mediated by 5hydroxytryptamine2C receptors. Journal of Pharmacology and Experimental Therapeutics, 295(3), 1120-1126.

Cryan, J. F., Page, M. E., \& Lucki, I. (2005). Differential behavioral effects of the antidepressants reboxetine, fluoxetine, and moclobemide in a modified forced swim test following chronic treatment. Psychopharmacology, 182(3), 335-344.

Cryan, J. F., Page, M. E., \& Lucki, I. (2005). Differential behavioral effects of the antidepressants reboxetine, fluoxetine, and moclobemide in a modified forced swim test following chronic treatment. Psychopharmacology, 182(3), 335-344.

Cui, Y., Cao, K., Lin, H., Cui, S., Shen, C., Wen, W., ... \& Zhang, R. (2020). Early-life stress induces depression-like behavior and synaptic-plasticity changes in a maternal separation rat model: gender difference and metabolomics study. Frontiers in pharmacology, 11, 102.

Dalla, C., Pitychoutis, P. M., Kokras, N., \& Papadopoulou-Daifoti, Z. (2010). Sex Differences in Animal Models of Depression and Antidepressant Response. Basic \& Clinical Pharmacology \& Toxicology, 106(3), 226-233.

Dawson, G. R., Crawford, S. P., Stanhope, K. J., Iversen, S. D., \& Tricklebank, M. D. (1994). One-trial tolerance to the effects of chlordiazepoxide on the elevated plus maze may be due to locomotor habituation, not repeated drug exposure. Psychopharmacology, $113(3), 570-572$.

De Pablo, J. M., Parra, A., Segovia, S., \& Guillamón, A. (1989). Learned immobility explains the behavior of rats in the forced swimming test. Physiology \& behavior, 46(2), 229237.

Deacon, R. M. J. (2013). The Successive Alleys Test of Anxiety in Mice and Rats. Journal of Visualized Experiments, 76, e2705. 
Delgado, P. L. (2000). Depression: the case for a monoamine deficiency. Journal of clinical Psychiatry, 61(6), 7-11.

Detke, M. J., Johnson, J., \& Lucki, I. (1997). Acute and chronic antidepressant drug treatment in the rat forced swimming test model of depression. Experimental and Clinical Psychopharmacology, 5(2), 107-112.

Detke, M. J., Johnson, J., \& Lucki, I. (1997). Acute and chronic antidepressant drug treatment in the rat forced swimming test model of depression. Experimental and Clinical Psychopharmacology, 5(2), 107-112.

Ecevitoglu, A., Canbeyli, R., \& Unal, G. (2019). Oral ketamine alleviates behavioral despair without cognitive impairment in Wistar rats. Behavioural brain research, 372, 112058.

Engin, E., Treit, D., \& Dickson, C. T. (2009). Anxiolytic- and antidepressant-like properties of ketamine in behavioral and neurophysiological animal models. Neuroscience, 161(2), $359-369$.

Ferguson, J. M. (2001). SSRI antidepressant medications: adverse effects and tolerability. Primary care companion to the Journal of clinical psychiatry, 3(1), 22.

Figlewicz, D., Higgins, M., Ng-Evans, S., \& Havel, P. (2001). Leptin reverses sucroseconditioned place preference in food-restricted rats. Physiology \& Behavior, 73(1-2), $229-234$.

File, S. E., \& Fernandes, C. (1994). Dizocilpine prevents the development of tolerance to the sedative effects of diazepam in rats. Pharmacology Biochemistry and Behavior, 47(4), 823-826

File, S. E., Mabbutt, P. S., \& Hitchcott, P. K. (1990). Characterisation of the phenomenon of "one-trial tolerance" to the anxiolytic effect of chlordiazepoxide in the elevated plusmaze. Psychopharmacology, 102(1), 98-101.

France, C. P., Li, J. X., Owens, W. A., Koek, W., Toney, G. M., \& Daws, L. C. (2009). Reduced effectiveness of escitalopram in the forced swimming test is associated with increased serotonin clearance rate in food-restricted rats. International Journal of Neuropsychopharmacology, 12(6), 731-736.

Freund, N., Thompson, B. S., Denormandie, J., Vaccarro, K., \& Andersen, S. L. (2013). Windows of vulnerability: maternal separation, age, and fluoxetine on adolescent depressive-like behavior in rats. Neuroscience, 249, 88-97.

Friedrich, M. J. (2017). Depression is the leading cause of disability around the world. Jama, 317(15), 1517-1517. 
Fuchs, E., \& Flügge, G. (2006). Experimental animal models for the simulation of depression and anxiety. Dialogues in Clinical Neuroscience, 8(3), 323-333.

Garcia, A. M. B., Cardenas, F. P., \& Morato, S. (2011). The effects of pentylenetetrazol, chlordiazepoxide and caffeine in rats tested in the elevated plus-maze depend on the experimental illumination. Behavioural Brain Research, 217(1), 171-177.

Garcia, L. S. B., Comim, C. M., Valvassori, S. S., Réus, G. Z., Barbosa, L. M., Andreazza, A. C., Stertz, L., Fries, G. R., Gavioli, E. C., Kapczinski, F., \& Quevedo, J. (2008). Acute administration of ketamine induces antidepressant-like effects in the forced swimming test and increases BDNF levels in the rat hippocampus. Progress in NeuroPsychopharmacology and Biological Psychiatry, 32(1), 140-144.

Glue, P., Neehoff, S., Sabadel, A., Broughton, L., Le Nedelec, M., Shadli, S., McNaughton, N., \& Medlicott, N. J. (2020). Effects of ketamine in patients with treatment-refractory generalized anxiety and social anxiety disorders: Exploratory double-blind psychoactive-controlled replication study. Journal of Psychopharmacology, 34(3), $267-272$.

Goltstein, P. M., Reinert, S., Glas, A., Bonhoeffer, T., \& Hübener, M. (2018). Food and water restriction lead to differential learning behaviors in a head-fixed two-choice visual discrimination task for mice. PLOS ONE, 13(9), e0204066.

Graf, E. N., Wheeler, R. A., Baker, D. A., Ebben, A. L., Hill, J. E., McReynolds, J. R., Robble, M. A., Vranjkovic, O., Wheeler, D. S., Mantsch, J. R., \& Gasser, P. J. (2013). Corticosterone Acts in the Nucleus Accumbens to Enhance Dopamine Signaling and Potentiate Reinstatement of Cocaine Seeking. The Journal of Neuroscience, 33(29), $11800-11810$.

Hau, J. (2008). Animal models for human diseases. In Conn P.M. (eds), Sourcebook of models for biomedical research (pp. 3-8). Humana Press.

Heiderstadt, K. M., McLaughlin, R. M., Wrighe, D. C., Walker, S. E., \& Gomez-Sanchez, C. E. (2000). The effect of chronic food and water restriction on open-field behaviour and serum corticosterone levels in rats. Laboratory animals, 34(1), 20-28.

Henniger, M. S., Ohl, F., Hölter, S. M., Weißenbacher, P., Toschi, N., Lörscher, P., ... \& Landgraf, R. (2000). Unconditioned anxiety and social behaviour in two rat lines selectively bred for high and low anxiety-related behaviour. Behavioural brain research, 111(1-2), 153-163.

Hill, M. N., Brotto, L. A., Lee, T. T. Y., \& Gorzalka, B. B. (2003). Corticosterone attenuates the antidepressant-like effects elicited by melatonin in the forced swim test in both male 
and female rats. Progress in Neuro-Psychopharmacology and Biological Psychiatry, 27(6), 905-911.

Homberg, J. R., De Boer, S. F., Raasø, H. S., Olivier, J. D. A., Verheul, M., Ronken, E., Cools, A. R., Ellenbroek, B. A., Schoffelmeer, A. N. M., Vanderschuren, L. J. M. J., De Vries, T. J., \& Cuppen, E. (2008). Adaptations in pre- and postsynaptic 5-HT1A receptor function and cocaine supersensitivity in serotonin transporter knockout rats. Psychopharmacology, 200(3), 367-380.

Houwing, D. J., Buwalda, B., van der Zee, E. A., de Boer, S. F., \& Olivier, J. D. (2017). The serotonin transporter and early life stress: translational perspectives. Frontiers in cellular neuroscience, 11, 117.

Houwing, D. J., Staal, L., Swart, J. M., Ramsteijn, A. S., Wöhr, M., de Boer, S. F., \& Olivier, J. D. A. (2019). Subjecting Dams to Early Life Stress and Perinatal Fluoxetine Treatment Differentially Alters Social Behavior in Young and Adult Rat Offspring. Frontiers in Neuroscience, 13, 229.

Hughes, J. E., Amyx, H., Howard, J. L., Nanry, K. P., \& Pollard, G. T. (1994). Health effects of water restriction to motivate lever-pressing in rats. Laboratory animal science, 44(2), 135-140.

Ibrahim, L., DiazGranados, N., Franco-Chaves, J., Brutsche, N., Henter, I. D., Kronstein, P., Moaddel, R., Wainer, I., Luckenbaugh, D. A., Manji, H. K., \& Zarate, C. A. (2012). Course of Improvement in Depressive Symptoms to a Single Intravenous Infusion of Ketamine vs Add-on Riluzole: Results from a 4-Week, Double-Blind, PlaceboControlled Study. Neuropsychopharmacology, 37(6), 1526-1533.

Iio, W., Tokutake, Y., Koike, H., Matsukawa, N., Tsukahara, T., Chohnan, S., \& Toyoda, A. (2015). Effects of chronic mild food restriction on behavior and the hypothalamic malonyl-CoA signaling pathway. Animal Science Journal, 86(2), 181-188.

Ishiyama, S., Kaufmann, L. V., \& Brecht, M. (2019). Behavioral and Cortical Correlates of Self-Suppression, Anticipation, and Ambivalence in Rat Tickling. Current Biology, 29(19), 3153-3164.

Jahng, J. W., Kim, J. G., Kim, H. J., Kim, B.-T., Kang, D.-W., \& Lee, J.-H. (2007). Chronic food restriction in young rats results in depression- and anxiety-like behaviors with decreased expression of serotonin reuptake transporter. Brain Research, 1150, 100107.

James, S. L., Abate, D., Abate, K. H., Abay, S. M., Abbafati, C., Abbasi, N., ... \& Briggs, A. M. (2018). Global, regional, and national incidence, prevalence, and years lived with 
disability for 354 diseases and injuries for 195 countries and territories, 1990-2017: a systematic analysis for the Global Burden of Disease Study 2017. The Lancet, 392(10159), 1789-1858.

Jiang, X., Wang, J., Luo, T., \& Li, Q. (2009). Impaired hypothalamic-pituitary-adrenal axis and its feedback regulation in serotonin transporter knockout mice. Psychoneuroendocrinology, 34(3), 317-331.

Jin, S., Zhao, Y., Jiang, Y., Wang, Y., Li, C., Zhang, D., ... \& Sun, L. (2018). Anxiety-like behaviour assessments of adolescent rats after repeated maternal separation during early life. Neuroreport, 29(8), 643.

Jonasson, Z. (2005). Meta-analysis of sex differences in rodent models of learning and memory: a review of behavioral and biological data. Neuroscience \& Biobehavioral Reviews, 28(8), 811-825.

Kant, G. J., Yen, M. H., D'Angelo, P. C., Brown, A. J., \& Eggleston, T. (1988). Maze performance: a direct comparison of food vs. water mazes. Pharmacology Biochemistry and Behavior, 31(2), 487-491.

Karg, K., Burmeister, M., Shedden, K., \& Sen, S. (2011). The Serotonin Transporter Promoter Variant (5-HTTLPR), Stress, and Depression Meta-analysis Revisited: Evidence of Genetic Moderation. Archives of General Psychiatry, 68(5), 444.

Keller, M. B., Lavori, P. W., Goldenberg, I. M., Baker, L. A., Pollack, M. H., Sachs, G. S., ... \& Klerman, G. L. (1993). Influence of depression on the treatment of panic disorder with imipramine, alprazolam and placebo. Journal of affective disorders, 28(1), 27-38.

Kennedy, B. L., Schwab, J. J., Morris, R. L., \& Beldia, G. (2001). Assessment of state and trait anxiety in subjects with anxiety and depressive disorders. Psychiatric Quarterly, 72(3), 263-276.

Kidwell, M. (2019). Establishment of an Animal Model of Depression: The Serotonin Transporter Knockout Rat (Master's thesis, Victoria University of Wellington). Victoria University of Wellington Research Archive.

Kirk, R. G. W. (2018). Recovering The Principles of Humane Experimental Technique: The 3Rs and the Human Essence of Animal Research. Science, Technology, \& Human Values, 43(4), 622-648.

Kisko, T. M., Wöhr, M., Pellis, V. C., \& Pellis, S. M. (2015). From play to aggression: highfrequency $50-\mathrm{kHz}$ ultrasonic vocalizations as play and appeasement signals in rats. Social Behavior from Rodents to Humans, 91-108. 
Knight, P., Chellian, R., Wilson, R., Behnood-Rod, A., Panunzio, S., \& Bruijnzeel, A. W. (2021). Sex differences in the elevated plus-maze test and large open field test in adult Wistar rats. Pharmacology Biochemistry and Behavior, 204, 173168.

Koike, H., Iijima, M., \& Chaki, S. (2013). Effects of ketamine and LY341495 on the depressive-like behavior of repeated corticosterone-injected rats. Pharmacology Biochemistry and Behavior, 107, 20-23.

Koot, S., van den Bos, R., Adriani, W., \& Laviola, G. (2009). Gender differences in delaydiscounting under mild food restriction. Behavioural Brain Research, 200(1), 134-143.

Koprdova, R., Bogi, E., Belovicova, K., Sedlackova, N., Okuliarová, M., Ujházy, E., \& Mach, M. (2016). Chronic unpredictable mild stress paradigm in male Wistar rats: effect on anxiety-and depressive-like behavior. Neuro Endocrinol Lett, 37(1), 103-110.

Kudagi, B., Kumar, R. P., \& Basha, S. S. (2012). Evaluation of anti-anxiety, sedative and motor co-ordination properties of ganaxolone in comparison with diazepam in rodent models. Journal of Dental and Medical Sciences, 1(4), 42-47.

Kwak, H. R., Lee, J. W., Kwon, K.-J., Kang, C. D., Cheong, I. Y., Chun, W., Kim, S.-S., \& Lee, H. J. (2009). Maternal Social Separation of Adolescent Rats Induces Hyperactivity and Anxiolytic Behavior. The Korean Journal of Physiology \& Pharmacology: Official Journal of the Korean Physiological Society and the Korean Society of Pharmacology, 13(2), 79-83.

Lapointe, J.-M., Snyder, P. A., \& Reagan, W. J. (2016). Evaluation of urinary corticosterone as a biomarker of stress in rats using fenitrothion as a chemical stressor. Journal of Immunotoxicology, 13(3), 386-392.

Lau, J., Eley, T., \& Stevenson, J. (2006). Examining the State-Trait Anxiety Relationship: A Behavioural Genetic Approach. Journal of Abnormal Child Psychology, 34, 19-27.

LeGates, T. A., Kvarta, M. D., \& Thompson, S. M. (2019). Sex differences in antidepressant efficacy. Neuropsychopharmacology, 44(1), 140-154.

Legrand, L. N., McGue, M., \& Iacono, W. G. (1999). Searching for interactive effects in the etiology of early-onset substance use. Behavior genetics, 29(6), 433-444.

Leussis, M. P., Freund, N., Brenhouse, H. C., Thompson, B. S., \& Andersen, S. L. (2012). Depressive-like behavior in adolescents after maternal separation: sex differences, controllability, and GABA. Developmental neuroscience, 34(2-3), 210-217.

Li, J. X., \& France, C. P. (2008). Food restriction and streptozotocin treatment decrease 5HT1A and 5-HT2A receptor mediated behavioral effects in rats. Behavioural pharmacology, 19(4), 292. 
Liebsch, G., Montkowski, A., Holsboer, F., \& Landgraf, R. (1998). Behavioural profiles of two Wistar rat lines selectively bred for high or low anxiety-related behaviour. Behavioural brain research, 94(2), 301-310.

Lira, A., Zhou, M., Castanon, N., Ansorge, M. S., Gordon, J. A., Francis, J. H., ... \& Gingrich, J. A. (2003). Altered depression-related behaviors and functional changes in the dorsal raphe nucleus of serotonin transporter-deficient mice. Biological psychiatry, 54(10), 960-971.

Ma, L., Xu, Y., Wang, G., \& Li, R. (2019). What do we know about sex differences in depression: A review of animal models and potential mechanisms. Progress in NeuroPsychopharmacology and Biological Psychiatry, 89, 48-56.

Ma, X.-C., Dang, Y.-H., Jia, M., Ma, R., Wang, F., Wu, J., Gao, C.-G., \& Hashimoto, K. (2013). Long-Lasting Antidepressant Action of Ketamine, but Not Glycogen Synthase Kinase-3 Inhibitor SB216763, in the Chronic Mild Stress Model of Mice. PLoS ONE, $8(2)$, e56053.

Makowiecki, K., Hammond, G., \& Rodger, J. (2012). Different levels of food restriction reveal genotype-specific differences in learning a visual discrimination task. PloS one, 7(11), e48703.

Makowska, I. J., \& Weary, D. M. (2016). Differences in Anticipatory Behaviour between Rats (Rattus norvegicus) Housed in Standard versus Semi-Naturalistic Laboratory Environments. PLOS ONE, 11(1), 1-20.

Mar, A., Spreekmeester, E., \& Rochford, J. (2002). Fluoxetine-induced increases in open-field habituation in the olfactory bulbectomized rat depend on test aversiveness but not on anxiety. Pharmacology Biochemistry and Behavior, 73(3), 703-712.

Marais, L., van Rensburg, S. J., van Zyl, J. M., Stein, D. J., \& Daniels, W. M. U. (2008). Maternal separation of rat pups increases the risk of developing depressive-like behavior after subsequent chronic stress by altering corticosterone and neurotrophin levels in the hippocampus. Neuroscience Research, 61(1), 106-112.

Marinelli, M., Le Moal, M., \& Piazza, P. V. (1996). Acute pharmacological blockade of corticosterone secretion reverses food restriction-induced sensitization of the locomotor response to cocaine. Brain research, 724(2), 251-255.

Masand, P. S. (2003). Tolerability and adherence issues in antidepressant therapy. Clinical therapeutics, 25(8), 2289-2304. 
Mathew, A. R., Pettit, J. W., Lewinsohn, P. M., Seeley, J. R., \& Roberts, R. E. (2011). Comorbidity between major depressive disorder and anxiety disorders: Shared etiology or direct causation? Psychological Medicine, 41(10), 2023-2034.

Mazzanti, C. M., Lappalainen, J., Long, J. C., Bengel, D., Naukkarinen, H., Eggert, M., Virkkunen, M., Linnoila, M., \& Goldman, D. (1998). Role of the Serotonin Transporter Promoter Polymorphism in Anxiety-Related Traits. Archives of General Psychiatry, $55(10), 936$.

McCormick, C. M., \& Green, M. R. (2013). From the stressed adolescent to the anxious and depressed adult: Investigations in rodent models. Neuroscience, 249, 242-257.

Mechan, A. O., Moran, P. M., Elliott, M. J., Young, A. M., Joseph, M. H., \& Green, R. A. (2002). A comparison between Dark Agouti and Sprague-Dawley rats in their behaviour on the elevated plus-maze, open-field apparatus and activity meters, and their response to diazepam. Psychopharmacology, 159(2), 188-195.

Mohammad, F., Ho, J., Woo, J. H., Lim, C. L., Poon, D. J. J., Lamba, B., \& Claridge-Chang, A. (2016). Concordance and incongruence in preclinical anxiety models: Systematic review and meta-analyses. Neuroscience \& Biobehavioral Reviews, 68, 504-529.

Molendijk, M. L., \& de Kloet, E. R. (2015). Immobility in the forced swim test is adaptive and does not reflect depression. Psychoneuroendocrinology, 62, 389-391.

Monteiro, S., Roque, S., de Sá-Calçada, D., Sousa, N., Correia-Neves, M., \& Cerqueira, J. J. (2015). An efficient chronic unpredictable stress protocol to induce stress-related responses in C57BL/6 mice. Frontiers in psychiatry, 6, 6.

Nakamura, M., Ueno, S., Sano, A., \& Tanabe, H. (2000). The human serotonin transporter gene linked polymorphism (5-HTTLPR) shows ten novel allelic variants. Molecular Psychiatry, 5(1), 32-38.

Neighbors, B., Kempton, T., \& Forehand, R. (1992). Co-occurence of substance abuse with conduct, anxiety, and depression disorders in juvenile delinquents. Addictive behaviors, 17(4), 379-386.

Nierenberg, A. A., Farabaugh, A. H., Alpert, J. E., Gordon, J., Worthington, J. J., Rosenbaum, J. F., \& Fava, M. (2000). Timing of onset of antidepressant response with fluoxetine treatment. American Journal of Psychiatry, 157(9), 1423-1428.

Nischal, A., Tripathi, A., Nischal, A., \& Trivedi, J. K. (2012). Suicide and antidepressants: what current evidence indicates. Mens sana monographs, 10(1), 33.

Nishimura, H., Tsuda, A., Oguchi, M., Ida, Y., \& Tanaka, M. (1988). Is immobility of rats in the forced swim test "behavioral despair?". Physiology \& behavior, 42(1), 93-95. 
O'Connell, A. C. (2020). The Developmental Role of Serotonin: A Comparison of Short-and Long-Term Blockade of the Serotonin Transporter on Behavioural Outcomes in Adulthood (Unpublished Master's thesis). Victoria University of Wellington.

OECD. (2019), Health at a Glance 2019: OECD Indicators

Olivier, J. D. A., Van Der Hart, M. G. C., Van Swelm, R. P. L., Dederen, P. J., Homberg, J. R., Cremers, T., Deen, P. M. T., Cuppen, E., Cools, A. R., \& Ellenbroek, B. A. (2008). A study in male and female 5-HT transporter knockout rats: An animal model for anxiety and depression disorders. Neuroscience, 152(3), 573-584.

Overstreet, D. H., Friedman, E., Mathé, A. A., \& Yadid, G. (2005). The Flinders Sensitive Line rat: A selectively bred putative animal model of depression. Neuroscience \& Biobehavioral Reviews, 29(4-5), 739-759.

Pacheco-Unguetti, A. P., Acosta, A., Callejas, A., \& Lupiáñez, J. (2010). Attention and anxiety: Different attentional functioning under state and trait anxiety. Psychological science, 21(2), 298-304.

Parise, E. M., Alcantara, L. F., Warren, B. L., Wright, K. N., Hadad, R., Sial, O. K., Kroeck, K. G., Iñiguez, S. D., \& Bolaños-Guzmán, C. A. (2013). Repeated Ketamine Exposure Induces an Enduring Resilient Phenotype in Adolescent and Adult Rats. Biological Psychiatry, 74(10), 750-759.

Paul, E. S., Harding, E. J., \& Mendl, M. (2005). Measuring emotional processes in animals: the utility of a cognitive approach. Neuroscience \& Biobehavioral Reviews, 29(3), 469491.

Pellow, S., Chopin, P., File, S. E., \& Briley, M. (1985). Validation of open: Closed arm entries in an elevated plus-maze as a measure of anxiety in the rat. Journal of Neuroscience Methods, 14(3), 149-167.

Pereira, J. K. D., Vieira, R. J., Konishi, C. T., de A. Ribeiro, R., \& Frussa-Filho, R. (1999). The phenomenon of "one-trial tolerance" to the anxiolytic effect of chlordiazepoxide in the elevated plus-maze is abolished by the introduction of a motivational conflict situation. Life Sciences, 65(10), 101-107.

Pigott, H. E., Leventhal, A. M., Alter, G. S., \& Boren, J. J. (2010). Efficacy and effectiveness of antidepressants: current status of research. Psychotherapy and psychosomatics, 79(5), 267-279.

Planchez, B., Surget, A., \& Belzung, C. (2019). Animal models of major depression: Drawbacks and challenges. Journal of Neural Transmission, 126(11), 1383-1408. 
Popik, P., Hołuj, M., Kos, T., Nowak, G., Librowski, T., \& Sałat, K. (2017). Comparison of the psychopharmacological effects of tiletamine and ketamine in rodents. Neurotoxicity research, 32(4), 544-554.

Porsolt, R. D., Le Pichon, M., \& Jalfre, M. (1977). Depression: A new animal model sensitive to antidepressant treatments. Nature, 266(5604), 730-732.

Pringle, A., Warren, M., Gottwald, J., Cowen, P. J., \& Harmer, C. J. (2016). Cognitive mechanisms of diazepam administration: a healthy volunteer model of emotional processing. Psychopharmacology, 233(12), 2221-2228.

Rajab, E., Alqanbar, B., Naiser, M. J., Abdulla, H. A., Al-Momen, M. M., \& Kamal, A. (2014). Sex differences in learning and memory following short-term dietary restriction in the rat. International Journal of Developmental Neuroscience, 36, 74-80.

Rex, A., Stephens, D. N., \& Fink, H. (1996). “Anxiolytic” action of diazepam and abecarnil in a modified open field test. Pharmacology Biochemistry and Behavior, 53(4), 10051011.

Rex, A., Voigt, J.-P., Gustedt, C., Beckett, S., \& Fink, H. (2004). Anxiolytic-like profile in Wistar, but not Sprague?Dawley rats in the social interaction test. Psychopharmacology, 177(1-2), 23-34.

Rizvi, S. J., Pizzagalli, D. A., Sproule, B. A., \& Kennedy, S. H. (2016). Assessing anhedonia in depression: Potentials and pitfalls. Neuroscience \& Biobehavioral Reviews, 65, 2135.

Roberge, M. C., Hotte-Bernard, J., Messier, C., \& Plamondon, H. (2008). Food restriction attenuates ischemia-induced spatial learning and memory deficits despite extensive CA1 ischemic injury. Behavioural brain research, 187(1), 123-132.

Rodgers, R. J., \& Shepherd, J. K. (1993). Influence of prior maze experience on behaviour and response to diazepam in the elevated plus-maze and light/dark tests of anxiety in mice. Psychopharmacology, 113(2), 237-242.

Saito, Y., Tachibana, R. O., \& Okanoya, K. (2019). Acoustical cues for perception of emotional vocalizations in rats. Scientific Reports, 9(1), 1-9.

Sakakibara, Y., Kasahara, Y., Hall, F. S., Lesch, K. P., Murphy, D. L., Uhl, G. R., \& Sora, I. (2014). Developmental alterations in anxiety and cognitive behavior in serotonin transporter mutant mice. Psychopharmacology, 231(21), 4119-4133.

Sanacora, G., Zarate, C. A., Krystal, J. H., \& Manji, H. K. (2008). Targeting the glutamatergic system to develop novel, improved therapeutics for mood disorders. Nature reviews Drug discovery, 7(5), 426-437. 
Schmitt, U., \& Hiemke, C. (1998). Combination of open field and elevated plus-maze: A suitable test battery to assess strain as well as treatment differences in rat behavior. Progress in Neuro-Psychopharmacology and Biological Psychiatry, 22(7), 1197-1215.

Schmitt, U., \& Hiemke, C. (1998). Combination of open field and elevated plus-maze: A suitable test battery to assess strain as well as treatment differences in rat behavior. Progress in Neuro-Psychopharmacology and Biological Psychiatry, 22(7), 1197-1215.

Selaković, D., \& Joksimović, J. (2014). Behavioural effects of short-term total food restriction in rats. Serbian Journal of Experimental and Clinical Research, 15(3), 129-137.

Sequeira-Cordero, A., Salas-Bastos, A., Fornaguera, J., \& Brenes, J. C. (2019). Behavioural characterisation of chronic unpredictable stress based on ethologically relevant paradigms in rats. Scientific reports, $9(1), 1-21$.

Serretti, A., Kato, M., De Ronchi, D., \& Kinoshita, T. (2007). Meta-analysis of serotonin transporter gene promoter polymorphism (5-HTTLPR) association with selective serotonin reuptake inhibitor efficacy in depressed patients. Molecular Psychiatry, 12(3), 247-257.

Sevak, R. J., Koek, W., Owens, W. A., Galli, A., Daws, L. C., \& France, C. P. (2008). Feeding conditions differentially affect the neurochemical and behavioral effects of dopaminergic drugs in male rats. European journal of pharmacology, 592(1-3), 109115.

Silvestre, J. S., Nadal, R., Pallarés, M., \& Ferré, N. (1997). Acute effects of ketamine in the holeboard, the elevated-plus maze, and the social interaction test in Wistar rats. Depression and Anxiety, 5(1), 29-33.

Simola, N., \& Granon, S. (2019). Ultrasonic vocalizations as a tool in studying emotional states in rodent models of social behavior and brain disease. Neuropharmacology, 159, 107420.

Slattery, D. A., \& Cryan, J. F. (2012). Using the rat forced swim test to assess antidepressantlike activity in rodents. Nature Protocols, 7(6), 1009-1014.

Smits, B. M. G., Mudde, J. B., van de Belt, J., Verheul, M., Olivier, J., Homberg, J., Guryev, V., Cools, A. R., Ellenbroek, B. A., Plasterk, R. H. A., \& Cuppen, E. (2006). Generation of gene knockouts and mutant models in the laboratory rat by ENU-driven targetselected mutagenesis. Pharmacogenetics and genomics, 16(3), 159-169.

Soubrié, P., Martin, P., Massol, J., \& Guadel, G. (1989). Attenuation of response to antidepressants in animals induced by reduction in food intake. Psychiatry research, 27(2), 149-159. 
Stephens, D. N., \& Schneider, H. H. (1985). Tolerance to the benzodiazepine diazepam in an animal model of anxiolytic activity. Psychopharmacology, 87(3), 322-327.

Steru, L., Chermat, R., Thierry, B., \& Simon, P. (1985). The tail suspension test: A new method for screening antidepressants in mice. Psychopharmacology, 85(3), 367-370.

Sturman, O., Germain, P. L., \& Bohacek, J. (2018). Exploratory rearing: a context-and stresssensitive behavior recorded in the open-field test. Stress, 21(5), 443-452.

Takahashi, N., Kashino, M., \& Hironaka, N. (2010). Structure of Rat Ultrasonic Vocalizations and Its Relevance to Behavior. PLoS ONE, 5(11), e14115.

Tasan, R. O., Bukovac, A., Peterschmitt, Y. N., Sartori, S. B., Landgraf, R., Singewald, N., \& Sperk, G. (2011). Altered GABA transmission in a mouse model of increased trait anxiety. Neuroscience, $183,71-80$.

Taylor, J. H., Landeros-Weisenberger, A., Coughlin, C., Mulqueen, J., Johnson, J. A., Gabriel, D., Reed, M. O., Jakubovski, E., \& Bloch, M. H. (2018). Ketamine for Social Anxiety Disorder: A Randomized, Placebo-Controlled Crossover Trial. Neuropsychopharmacology, 43(2), 325-333.

Thomas, R., Urban, J., \& Peterson, D. (2006). Acute exposure to predator odor elicits a robust increase in corticosterone and a decrease in activity without altering proliferation in the adult rat hippocampus. Experimental Neurology, 201(2), 308-315.

Thomsen, K. R. (2015). Measuring anhedonia: Impaired ability to pursue, experience, and learn about reward. Frontiers in Psychology, 6, 1409

Toth, L. A., \& Gardiner, T. W. (2000). Food and Water Restriction Protocols: Physiological and Behavioral Considerations. 39(6), 9-17.

Treadway, M. T., Bossaller, N. A., Shelton, R. C., \& Zald, D. H. (2012). Effort-based decisionmaking in major depressive disorder: a translational model of motivational anhedonia. Journal of abnormal psychology, 121(3), 553-558

Treit, D., Menard, J., \& Royan, C. (1993). Anxiogenic stimuli in the elevated plus-maze. Pharmacology Biochemistry and Behavior, 44(2), 463-469.

Ulloa, J. L., Castañeda, P., Berríos, C., Díaz-Veliz, G., Mora, S., Bravo, J. A., ... \& Fiedler, J. L. (2010). Comparison of the antidepressant sertraline on differential depression-like behaviors elicited by restraint stress and repeated corticosterone administration. Pharmacology Biochemistry and Behavior, 97(2), 213-221.

Verdolin, J. L. (2006). Meta-analysis of foraging and predation risk trade-offs in terrestrial systems. Behavioral Ecology and Sociobiology, 60(4), 457-464. 
Viana, G. S. B., Vale, E. M. do, Araujo, A. R. A. de, Coelho, N. C., Andrade, S. M., Costa, R. O. da, Aquino, P. E. A. de, Sousa, C. N. S. de, Medeiros, I. S. de, Vasconcelos, S. M. M. de, \& Neves, K. R. T. (2021). Rapid and long-lasting antidepressant-like effects of ketamine and their relationship with the expression of brain enzymes, BDNF, and astrocytes. Brazilian Journal of Medical and Biological Research, 54(2), e10107.

Walf, A. A., \& Frye, C. A. (2007). The use of the elevated plus maze as an assay of anxietyrelated behavior in rodents. Nature Protocols, 2(2), 322-328.

Wang, D., Levine, J. L. S., Avila-Quintero, V., Bloch, M., \& Kaffman, A. (2020). Systematic review and meta-analysis: Effects of maternal separation on anxiety-like behavior in rodents. Translational Psychiatry, 10(1), 1-12.

Wang, J., Goffer, Y., Xu, D., Tukey, D. S., Shamir, D. B., Eberle, S. E., Zou, A. H., Blanck, T. J. J., \& Ziff, E. B. (2011). A Single Subanesthetic Dose of Ketamine Relieves Depression-like Behaviors Induced by Neuropathic Pain in Rats. Anesthesiology, $115(4), 812-821$.

Wendler, E., de Souza, C. P., Vecchia, D. D., Kanazawa, L. K. S., de Almeida Soares Hocayen, P., Wöhr, M., Schwarting, R. K. W., \& Andreatini, R. (2016). Evaluation of 50-kHz ultrasonic vocalizations in animal models of mania: Ketamine and lisdexamfetamineinduced hyperlocomotion in rats. European Neuropsychopharmacology, 26(12), 19001908.

Whitton, A. E., Treadway, M. T., \& Pizzagalli, D. A. (2015). Reward processing dysfunction in major depression, bipolar disorder and schizophrenia. Current opinion in psychiatry, 28(1), 7-12

Willner, P. (1984). The validity of animal models of depression. Psychopharmacology, 83(1), $1-16$.

Wilson, M. A., Burghardt, P. R., Ford, K. A., Wilkinson, M. B., \& Primeaux, S. D. (2004). Anxiolytic effects of diazepam and ethanol in two behavioral models: Comparison of males and females. Pharmacology Biochemistry and Behavior, 78(3), 445-458.

Winters BD, Saksida LM, Bussey TJ (2008). Object recognition

Wright, I. K., Heaton, M., Upton, N., \& Marsden, C. A. (1992). Comparison of acute and chronic treatment of various serotonergic agents with those of diazepam and idazoxan in the rat elevated X-maze. Psychopharmacology, 107(2-3), 405-414.

Yang, C., Hu, Y.-M., Zhou, Z.-Q., Zhang, G.-F., \& Yang, J.-J. (2013). Acute administration of ketamine in rats increases hippocampal BDNF and mTOR levels during forced swimming test. Upsala Journal of Medical Sciences, 118(1), 3-8. 
Yohn, N. L., \& Blendy, J. A. (2017). Adolescent chronic unpredictable stress exposure is a sensitive window for long-term changes in adult behavior in mice. Neuropsychopharmacology, 42(8), 1670-1678.

Zarate, C. A., Singh, J. B., Carlson, P. J., Brutsche, N. E., Ameli, R., Luckenbaugh, D. A., ... \& Manji, H. K. (2006). A randomized trial of an N-methyl-D-aspartate antagonist in treatment-resistant major depression. Archives of general psychiatry, 63(8), 856-864. 


\section{Appendix A}

Means and Standard Deviations for Proportion of 50kHz USVs (\%)

\begin{tabular}{|c|c|c|c|c|c|c|c|c|c|c|c|}
\hline Genotype & Treatment & & Day 1 & Day 2 & Day 3 & Day 4* & Day 5 & Day 6 & Day 7 & Day 8 & Day 9 \\
\hline \multirow{2}{*}{ WT } & & Mean & 49.78 & 57.61 & 57.47 & 58.26 & & & & & \\
\hline & & $S D$ & 38.68 & 34.55 & 34.37 & 30.69 & & & & & \\
\hline \multirow{2}{*}{ SERT $\%$} & & Mean & 33.26 & 37.11 & 45.22 & 50.35 & & & & & \\
\hline & & $S D$ & 30.37 & 27.41 & 29.51 & 28.78 & & & & & \\
\hline \multirow{6}{*}{ WT } & \multirow{2}{*}{ Saline } & Mean & & & & 55.11 & 55.06 & 49.38 & 46.36 & 56.78 & 61.62 \\
\hline & & $S D$ & & & & 36.31 & 41.10 & 37.75 & 36.88 & 40.95 & 42.35 \\
\hline & \multirow{2}{*}{ Diazepam } & Mean & & & & 66.21 & 9.76 & 78.47 & 57.23 & 65.21 & 46.07 \\
\hline & & $S D$ & & & & 30.22 & 12.20 & 29.06 & 29.69 & 24.02 & 37.17 \\
\hline & \multirow{2}{*}{ Ketamine } & Mean & & & & 53.08 & 49.91 & 65.39 & 67.40 & 56.43 & 60.58 \\
\hline & & $S D$ & & & & 28.35 & 32.90 & 32.93 & 31.63 & 37.13 & 33.54 \\
\hline \multirow{6}{*}{ SERT $\%$} & \multirow{2}{*}{ Saline } & Mean & & & & 45.47 & 59.34 & 39.25 & 42.64 & 33.90 & 49.38 \\
\hline & & $S D$ & & & & 34.72 & 40.13 & 38.39 & 39.56 & 43.10 & 35.86 \\
\hline & \multirow{2}{*}{ Diazepam } & Mean & & & & 52.32 & 47.36 & 63.75 & 39.95 & 49.54 & 41.03 \\
\hline & & $S D$ & & & & 24.37 & 33.62 & 38.34 & 22.47 & 35.42 & 21.98 \\
\hline & \multirow{2}{*}{ Ketamine } & Mean & & & & 53.68 & 46.99 & 44.78 & 59.27 & 61.80 & 67.90 \\
\hline & & $S D$ & & & & 29.79 & 26.8 & 25.27 & 31.08 & 28.67 & 30.42 \\
\hline
\end{tabular}

Note. ${ }^{*}$ Subjects were untreated on day 4, descriptive statistics from each treatment group on this day are provided to indicate pre-post treatment differences. 


\section{Appendix B}

Means and Standard Deviations for Rearing Behaviour

\begin{tabular}{|c|c|c|c|c|c|c|c|c|c|c|c|}
\hline Genotype & Treatment & & Day 1 & Day 2 & Day 3 & Day 4* & Day 5 & Day 6 & Day 7 & Day 8 & Day 9 \\
\hline \multirow{2}{*}{ WT } & & Mean & 20.70 & 24.74 & 25.70 & 33.96 & & & & & \\
\hline & & $S D$ & 7.91 & 7.53 & 10.48 & 10.44 & & & & & \\
\hline \multirow{2}{*}{ SERT $^{\%}$} & & Mean & 15.50 & 18.67 & 16.25 & 21.29 & & & & & \\
\hline & & $S D$ & 7.33 & 9.88 & 7.91 & 7.17 & & & & & \\
\hline \multirow{6}{*}{ WT } & \multirow{2}{*}{ Saline } & Mean & & & & 38.00 & 36.57 & 44.86 & 46.57 & 47.14 & 44.71 \\
\hline & & $S D$ & & & & 6.56 & 9.29 & 15.51 & 14.56 & 12.72 & 11.98 \\
\hline & \multirow{2}{*}{ Diazepam } & Mean & & & & 33.38 & 9.50 & 34.13 & 36.13 & 41.50 & 39.13 \\
\hline & & $S D$ & & & & 13.16 & 10.13 & 8.44 & 12.72 & 6.89 & 13.54 \\
\hline & \multirow{2}{*}{ Ketamine } & Mean & & & & 31.00 & 25.25 & 34.00 & 41.38 & 44.00 & 41.38 \\
\hline & & $S D$ & & & & 10.28 & 19.10 & 10.47 & 17.51 & 12.49 & 9.21 \\
\hline \multirow{6}{*}{ SERT $\%$} & \multirow{2}{*}{ Saline } & Mean & & & & 22.38 & 21.63 & 23.75 & 25.38 & 27.63 & 23.88 \\
\hline & & $S D$ & & & & 4.53 & 5.34 & 7.27 & 9.62 & 7.35 & 8.95 \\
\hline & \multirow{2}{*}{ Diazepam } & Mean & & & & 17.78 & 12.78 & 17.89 & 18.22 & 27.11 & 22.44 \\
\hline & & $S D$ & & & & 7.36 & 8.94 & 7.79 & 5.87 & 9.92 & 3.09 \\
\hline & \multirow{2}{*}{ Ketamine } & Mean & & & & 24.57 & 38.14 & 36.14 & 43.00 & 41.86 & 46.00 \\
\hline & & $S D$ & & & & 8.24 & 16.15 & 7.15 & 11.46 & 19.33 & 10.49 \\
\hline
\end{tabular}

Note. *Subjects were untreated on day 4, descriptive statistics from each treatment group on this day are provided to indicate pre-post treatment differences. 


\section{Appendix C}

Means and Standard Deviations for Cumulative Duration in Alley 4 (s)

\begin{tabular}{|c|c|c|c|c|c|c|c|c|c|c|c|}
\hline Genotype & Treatment & & Day 1 & Day 2 & Day 3 & Day 4* & Day 5 & Day 6 & Day 7 & Day 8 & Day 9 \\
\hline \multirow{2}{*}{ WT } & & Mean & 7.99 & 20.23 & 31.26 & 60.85 & & & & & \\
\hline & & $S D$ & 13.33 & 20.66 & 22.35 & 28.15 & & & & & \\
\hline \multirow{2}{*}{ SERT $\%$} & & Mean & 4.24 & 10.44 & 17.64 & 36.99 & & & & & \\
\hline & & $S D$ & 10.09 & 11.89 & 18.58 & 27.91 & & & & & \\
\hline \multirow{6}{*}{ WT } & \multirow{2}{*}{ Saline } & Mean & & & & 62.43 & 71.22 & 74.59 & 57.51 & 91.27 & 69.90 \\
\hline & & $S D$ & & & & 33.40 & 23.09 & 23.31 & 19.34 & 39.77 & 48.36 \\
\hline & \multirow{2}{*}{ Diazepam } & Mean & & & & 66.26 & 85.62 & 51.06 & 83.72 & 55.42 & 88.59 \\
\hline & & $S D$ & & & & 22.91 & 35.90 & 22.41 & 34.58 & 27.06 & 43.94 \\
\hline & \multirow{2}{*}{ Ketamine } & Mean & & & & 54.05 & 57.83 & 62.95 & 71.31 & 82.42 & 88.27 \\
\hline & & $S D$ & & & & 30.33 & 30.70 & 40.71 & 18.41 & 27.19 & 53.14 \\
\hline \multirow{6}{*}{ SERT $\%$} & \multirow{2}{*}{ Saline } & Mean & & & & 33.81 & 46.19 & 70.22 & 61.48 & 43.96 & 57.03 \\
\hline & & $S D$ & & & & 28.20 & 31.09 & 37.55 & 34.69 & 18.81 & 28.93 \\
\hline & \multirow{2}{*}{ Diazepam } & Mean & & & & 45.99 & 45.59 & 29.20 & 31.52 & 58.44 & 66.24 \\
\hline & & $S D$ & & & & 31.31 & 23.58 & 18.48 & 23.53 & 34.52 & 33.66 \\
\hline & \multirow{2}{*}{ Ketamine } & Mean & & & & 29.04 & 45.33 & 66.74 & 72.25 & 55.70 & 80.46 \\
\hline & & $S D$ & & & & 23.30 & 30.74 & 31.49 & 38.91 & 59.06 & 30.80 \\
\hline
\end{tabular}

Note. *Subjects were untreated on day 4, descriptive statistics from each treatment group on this day are provided to indicate pre-post treatment differences. 


\section{Appendix D}

Means and Standard Deviations for Latency to Enter Alley 4 (s)

\begin{tabular}{|c|c|c|c|c|c|c|c|c|c|c|c|}
\hline Genotype & Treatment & & Day 1 & Day 2 & Day 3 & Day 4* & Day 5 & Day 6 & Day 7 & Day 8 & Day 9 \\
\hline \multirow{2}{*}{ WT } & & Mean & 248.22 & 199.40 & 150.38 & 103.47 & & & & & \\
\hline & & $S D$ & 75.76 & 83.15 & 75.06 & 51.36 & & & & & \\
\hline \multirow{2}{*}{ SERT $\%$} & & Mean & 260.53 & 205.74 & 179.60 & 130.08 & & & & & \\
\hline & & $S D$ & 84.01 & 96.91 & 86.07 & 81.54 & & & & & \\
\hline \multirow{6}{*}{ WT } & \multirow{2}{*}{ Saline } & Mean & & & & 107.65 & 112.78 & 73.73 & 74.18 & 69.90 & 68.26 \\
\hline & & $S D$ & & & & 40.86 & 29.08 & 15.54 & 18.98 & 21.71 & 24.00 \\
\hline & \multirow{2}{*}{ Diazepam } & Mean & & & & 85.43 & 80.30 & 72.88 & 75.03 & 61.38 & 54.70 \\
\hline & & $S D$ & & & & 38.81 & 26.13 & 28.31 & 40.27 & 15.29 & 23.11 \\
\hline & \multirow{2}{*}{ Ketamine } & Mean & & & & 117.86 & 90.29 & 101.71 & 69.39 & 57.86 & 61.96 \\
\hline & & $S D$ & & & & 68.77 & 16.70 & 53.52 & 17.95 & 10.18 & 12.00 \\
\hline \multirow{6}{*}{ SERT $\%$} & \multirow{2}{*}{ Saline } & Mean & & & & 133.70 & 106.44 & 80.26 & 92.88 & 76.40 & 70.23 \\
\hline & & $S D$ & & & & 84.63 & 25.87 & 28.79 & 50.92 & 20.79 & 21.20 \\
\hline & \multirow{2}{*}{ Diazepam } & Mean & & & & 133.65 & 82.75 & 122.16 & 99.04 & 68.47 & 72.03 \\
\hline & & $S D$ & & & & 82.29 & 58.53 & 84.00 & 75.86 & 22.86 & 14.79 \\
\hline & \multirow{2}{*}{ Ketamine } & Mean & & & & 121.35 & 85.48 & 76.48 & 64.65 & 82.42 & 57.68 \\
\hline & & $S D$ & & & & 89.33 & 26.28 & 20.84 & 22.84 & 41.70 & 14.08 \\
\hline
\end{tabular}

Note. *Subjects were untreated on day 4, descriptive statistics from each treatment group on this day are provided to indicate pre-post treatment differences. 


\section{Appendix E}

Means and Standard Deviations for Cumulative Duration in Alley 3 (s)

\begin{tabular}{|c|c|c|c|c|c|c|c|c|c|c|c|}
\hline Genotype & Treatment & & Day 1 & Day 2 & Day 3 & Day 4* & Day 5 & Day 6 & Day 7 & Day 8 & Day 9 \\
\hline \multirow{2}{*}{ WT } & & Mean & 36.62 & 45.83 & 50.24 & 47.07 & & & & & \\
\hline & & $S D$ & 23.15 & 20.07 & 18.53 & 15.49 & & & & & \\
\hline \multirow{2}{*}{ SERT $\%$} & & Mean & 18.02 & 29.87 & 45.13 & 49.95 & & & & & \\
\hline & & $S D$ & 21.34 & 22.53 & 24.33 & 24.01 & & & & & \\
\hline \multirow{6}{*}{ WT } & \multirow{2}{*}{ Saline } & Mean & & & & 48.32 & 43.36 & 46.61 & 57.47 & 53.18 & 52.35 \\
\hline & & $S D$ & & & & 22.78 & 16.63 & 27.11 & 18.36 & 24.71 & 17.90 \\
\hline & \multirow{2}{*}{ Diazepam } & Mean & & & & 44.12 & 76.13 & 43.36 & 44.33 & 44.95 & 54.29 \\
\hline & & $S D$ & & & & 10.73 & 29.84 & 15.80 & 19.44 & 21.77 & 24.76 \\
\hline & \multirow{2}{*}{ Ketamine } & Mean & & & & 48.92 & 46.81 & 45.09 & 47.02 & 47.08 & 47.17 \\
\hline & & $S D$ & & & & 13.38 & 24.66 & 16.66 & 19.37 & 13.81 & 17.54 \\
\hline \multirow{6}{*}{ SERT \% } & \multirow{2}{*}{ Saline } & Mean & & & & 42.90 & 40.25 & 48.17 & 48.73 & 55.67 & 46.83 \\
\hline & & $S D$ & & & & 16.70 & 10.50 & 16.66 & 23.19 & 14.70 & 19.14 \\
\hline & \multirow{2}{*}{ Diazepam } & Mean & & & & 44.59 & 59.36 & 36.41 & 37.87 & 39.24 & 48.36 \\
\hline & & $S D$ & & & & 23.88 & 38.60 & 20.89 & 29.33 & 15.90 & 26.36 \\
\hline & \multirow{2}{*}{ Ketamine } & Mean & & & & 64.90 & 51.23 & 46.33 & 37.55 & 48.32 & 53.47 \\
\hline & & $S D$ & & & & 27.45 & 23.97 & 27.22 & 11.03 & 24.45 & 22.20 \\
\hline
\end{tabular}

Note. $*$ Subjects were untreated on day 4, descriptive statistics from each treatment group on this day are provided to indicate pre-post treatment differences. 


\section{Appendix F}

Means and Standard Deviations for Latency to Enter Alley 3 (s)

\begin{tabular}{|c|c|c|c|c|c|c|c|c|c|c|c|}
\hline Genotype & Treatment & & Day 1 & Day 2 & Day 3 & Day 4* & Day 5 & Day 6 & Day 7 & Day 8 & Day 9 \\
\hline \multirow{2}{*}{ WT } & & Mean & 113.94 & 97.72 & 70.57 & 52.08 & & & & & \\
\hline & & $S D$ & 82.80 & 73.04 & 33.77 & 34.63 & & & & & \\
\hline \multirow{2}{*}{ SERT $\%$} & & Mean & 159.75 & 129.60 & 74.99 & 57.71 & & & & & \\
\hline & & $S D$ & 123.23 & 102.55 & 57.41 & 42.53 & & & & & \\
\hline \multirow{6}{*}{ WT } & \multirow{2}{*}{ Saline } & Mean & & & & 64.81 & 58.31 & 39.56 & 38.17 & 36.40 & 37.65 \\
\hline & & $S D$ & & & & 52.21 & 10.54 & 6.77 & 10.17 & 12.34 & 12.87 \\
\hline & \multirow{2}{*}{ Diazepam } & Mean & & & & 53.05 & 43.71 & 36.19 & 36.54 & 34.53 & 28.03 \\
\hline & & $S D$ & & & & 28.66 & 10.67 & 23.80 & 19.01 & 12.77 & 12.56 \\
\hline & \multirow{2}{*}{ Ketamine } & Mean & & & & 39.97 & 47.18 & 54.00 & 36.88 & 31.83 & 37.08 \\
\hline & & $S D$ & & & & 16.76 & 14.71 & 25.02 & 11.64 & 7.14 & 6.34 \\
\hline \multirow{6}{*}{ SERT $\%$} & \multirow{2}{*}{ Saline } & Mean & & & & 50.32 & 43.57 & 40.41 & 34.68 & 43.94 & 38.81 \\
\hline & & $S D$ & & & & 25.61 & 11.59 & 23.48 & 15.21 & 11.87 & 11.68 \\
\hline & \multirow{2}{*}{ Diazepam } & Mean & & & & 74.96 & 47.93 & 61.61 & 44.84 & 39.65 & 31.19 \\
\hline & & $S D$ & & & & 57.48 & 36.50 & 56.09 & 9.64 & 16.34 & 14.66 \\
\hline & \multirow{2}{*}{ Ketamine } & Mean & & & & 43.97 & 42.86 & 47.52 & 36.76 & 46.51 & 22.37 \\
\hline & & $S D$ & & & & 31.98 & 18.36 & 13.28 & 29.39 & 14.97 & 16.79 \\
\hline
\end{tabular}

Note. $*$ Subjects were untreated on day 4, descriptive statistics from each treatment group on this day are provided to indicate pre-post treatment differences. 


\section{Appendix G}

Means and Standard Deviations for Retrieval Score

\begin{tabular}{|c|c|c|c|c|c|c|c|c|c|c|c|}
\hline Genotype & Treatment & & Day 1 & Day 2 & Day 3 & Day $4 *$ & Day 5 & Day 6 & Day 7 & Day 8 & Day 9 \\
\hline \multirow{2}{*}{ WT } & & Mean & 3.39 & 4.52 & 5.26 & 5.39 & & & & & \\
\hline & & $S D$ & 2.04 & 2.13 & 1.57 & 1.31 & & & & & \\
\hline \multirow{2}{*}{ SERT \% } & & Mean & 2.58 & 3.71 & 3.87 & 4.21 & & & & & \\
\hline & & $S D$ & 1.59 & 1.30 & 1.52 & 1.25 & & & & & \\
\hline \multirow{6}{*}{ WT } & \multirow{2}{*}{ Saline } & Mean & & & & 5.57 & 4.86 & 5.29 & 5.57 & 5.43 & 5.43 \\
\hline & & $S D$ & & & & 1.51 & 0.90 & 2.21 & 2.07 & 1.99 & 2.15 \\
\hline & \multirow{2}{*}{ Diazepam } & Mean & & & & 5.63 & 8.88 & 5.63 & 5.5 & 5.88 & 5.88 \\
\hline & & $S D$ & & & & 1.19 & 1.64 & 1.41 & 1.51 & 1.46 & 1.36 \\
\hline & \multirow{2}{*}{ Ketamine } & Mean & & & & 5.00 & 5.00 & 4.50 & 4.38 & 4.75 & 4.50 \\
\hline & & $S D$ & & & & 1.31 & 1.77 & 1.41 & 1.06 & 1.04 & 0.76 \\
\hline \multirow{6}{*}{ SERT ^ } & \multirow{2}{*}{ Saline } & Mean & & & & 4.00 & 4.00 & 4.00 & 4.13 & 4.00 & 4.38 \\
\hline & & $S D$ & & & & 0.54 & 0.00 & 0.54 & 0.641 & 0.00 & 0.74 \\
\hline & \multirow{2}{*}{ Diazepam } & Mean & & & & 4.11 & 7.33 & 4.78 & 4.00 & 4.67 & 4.78 \\
\hline & & $S D$ & & & & 1.54 & 1.58 & 1.30 & 0.5 & 1.00 & 1.09 \\
\hline & \multirow{2}{*}{ Ketamine } & Mean & & & & 4.57 & 4.86 & 5.00 & 5.29 & 5.57 & 5.57 \\
\hline & & $S D$ & & & & 1.51 & 2.34 & 1.53 & 2.21 & 2.07 & 2.07 \\
\hline
\end{tabular}

Note. *Subjects were untreated on day 4, descriptive statistics from each treatment group on this day are provided to indicate pre-post treatment differences. 


\section{Appendix H}

Means and Standard Deviations for Retrieval Latency (s)

\begin{tabular}{|c|c|c|c|c|c|c|c|c|c|c|c|}
\hline Genotype & Treatment & & Day 1 & Day 2 & Day 3 & Day 4* & Day 5 & Day 6 & Day 7 & Day 8 & Day 9 \\
\hline \multirow{2}{*}{ WT } & & Mean & 130.96 & 56.35 & 36.48 & 13.35 & & & & & \\
\hline & & $S D$ & 81.80 & 72.03 & 53.85 & 14.39 & & & & & \\
\hline \multirow{2}{*}{ SERT $\%$} & & Mean & 148.42 & 39.21 & 33.30 & 12.17 & & & & & \\
\hline & & $S D$ & 53.35 & 21.32 & 61.52 & 15.16 & & & & & \\
\hline \multirow{6}{*}{ WT } & \multirow{2}{*}{ Saline } & Mean & & & & 21.14 & 6.71 & 2.43 & 4.14 & 6.43 & 4.29 \\
\hline & & $S D$ & & & & 19.43 & 5.15 & 1.13 & 2.12 & 5.74 & 2.36 \\
\hline & \multirow{2}{*}{ Diazepam } & Mean & & & & 12.88 & 4.50 & 3.38 & 5.13 & 4.38 & 3.75 \\
\hline & & $S D$ & & & & 12.80 & 2.56 & 1.77 & 3.94 & 3.25 & 1.39 \\
\hline & \multirow{2}{*}{ Ketamine } & Mean & & & & 7.00 & 4.38 & 3.38 & 3.88 & 3.75 & 3.00 \\
\hline & & $S D$ & & & & 7.52 & 2.20 & 1.69 & 2.80 & 1.67 & 1.41 \\
\hline \multirow{6}{*}{ SERT $\%$} & \multirow{2}{*}{ Saline } & Mean & & & & 10.13 & 9.13 & 5.63 & 7.00 & 4.75 & 4.25 \\
\hline & & $S D$ & & & & 5.33 & 8.68 & 3.78 & 11.46 & 2.82 & 1.49 \\
\hline & \multirow{2}{*}{ Diazepam } & Mean & & & & 8.11 & 7.56 & 5.11 & 5.11 & 5.89 & 7.67 \\
\hline & & $S D$ & & & & 4.54 & 3.43 & 3.76 & 3.41 & 3.41 & 5.70 \\
\hline & \multirow{2}{*}{ Ketamine } & Mean & & & & 19.71 & 8.43 & 7.29 & 13.71 & 3.14 & 4.14 \\
\hline & & $S D$ & & & & 26.91 & 11.62 & 8.01 & 29.28 & 2.41 & 3.24 \\
\hline
\end{tabular}

Note. $*$ Subjects were untreated on day 4, descriptive statistics from each treatment group on this day are provided to indicate pre-post treatment differences. 


\section{Appendix I}

Means and Standard Deviations for Locomotor Activity (cm)

\begin{tabular}{|c|c|c|c|c|c|c|c|c|c|c|c|}
\hline Genotype & Treatment & & Day 1 & Day 2 & Day 3 & Day $4 *$ & Day 5 & Day 6 & Day 7 & Day 8 & Day 9 \\
\hline \multirow{2}{*}{ WT } & & Mean & 1071.23 & 1077.62 & 1154.75 & 1381.25 & & & & & \\
\hline & & $S D$ & 246.38 & 378.32 & 350.29 & 333.36 & & & & & \\
\hline \multirow{2}{*}{$\mathrm{SERT}^{\%}$} & & Mean & 1082.82 & 1001.89 & 1054.10 & 1379.22 & & & & & \\
\hline & & $S D$ & 326.03 & 489.20 & 356.74 & 338.21 & & & & & \\
\hline \multirow{6}{*}{ WT } & \multirow{2}{*}{ Saline } & Mean & & & & 1509.65 & 1397.45 & 1570.02 & 1866.96 & 1588.73 & 1647.97 \\
\hline & & $S D$ & & & & 320.16 & 355.40 & 406.79 & 679.39 & 386.80 & 460.48 \\
\hline & \multirow{2}{*}{ Diazepam } & Mean & & & & 1353.25 & 498.97 & 1512.32 & 1528.60 & 1448.63 & 1446.28 \\
\hline & & $S D$ & & & & 269.00 & 253.11 & 204.40 & 224.96 & 352.90 & 444.03 \\
\hline & \multirow{2}{*}{ Ketamine } & Mean & & & & 1266.50 & 1100.96 & 1319.61 & 1567.98 & 1584.98 & 1402.76 \\
\hline & & $S D$ & & & & 406.28 & 373.38 & 429.30 & 538.86 & 603.30 & 379.69 \\
\hline \multirow{6}{*}{ SERT $\%$} & \multirow{2}{*}{ Saline } & Mean & & & & 1444.30 & 1375.78 & 1375.42 & 1620.30 & 1594.66 & 1480.46 \\
\hline & & $S D$ & & & & 335.19 & 338.87 & 439.00 & 532.95 & 323.78 & 608.44 \\
\hline & \multirow{2}{*}{ Diazepam } & Mean & & & & 1184.10 & 1020.61 & 1026.15 & 1058.39 & 1358.71 & 1233.98 \\
\hline & & $S D$ & & & & 256.02 & 470.66 & 329.19 & 279.39 & 281.00 & 275.35 \\
\hline & \multirow{2}{*}{ Ketamine } & Mean & & & & 1527.82 & 1707.21 & 1664.90 & 1806.62 & 1788.23 & 2028.93 \\
\hline & & $S D$ & & & & 360.13 & 277.81 & 352.04 & 228.92 & 640.80 & 482.33 \\
\hline
\end{tabular}

Note. *Subjects were untreated on day 4, descriptive statistics from each treatment group on this day are provided to indicate pre-post treatment differences. 